\title{
The second and third parameters of the horizontal branch in globular clusters ${ }^{\star}$
}

\author{
R. G. Gratton ${ }^{1}$, E. Carretta ${ }^{2}$, A. Bragaglia ${ }^{2}$, S. Lucatello ${ }^{1,3}$, and V. D’Orazi ${ }^{1}$ \\ 1 INAF - Osservatorio Astronomico di Padova, Vicolo dell'Osservatorio 5, 35122 Padova, Italy \\ e-mail: [raffaele.gratton; sara.lucatello; valentina.dorazi]@oapd.inaf.it \\ 2 INAF - Osservatorio Astronomico di Bologna, via Ranzani 1, 40127 Bologna, Italy \\ e-mail: [angela.bragaglia; eugenio.carretta]@oabo.inaf.it \\ 3 Excellence Cluster Universe, Technische Universität München, Boltzmannstr. 2, 85748 Garching, Germany
}

Received 26 May 2009 / Accepted 17 April 2010

ABSTRACT

Context. The second parameter (the first being metallicity) defining the distribution of stars on the horizontal branch (HB) of globular clusters (GCs) has long been one of the major open issues in our understanding of the evolution of normal stars. Large photometric and spectroscopic databases are now available: they include large and homogeneous sets of colour-magnitude diagrams, cluster ages, and homogeneous data about chemical compositions from our FLAMES survey.

Aims. We use these databases to re-examine this issue.

Methods. We use the photometric data to derive median and extreme (i.e., the values including $90 \%$ of the distribution) colours and magnitudes of stars along the HB for about a hundred GCs. We transform these into median and extreme masses of stars on the HB, using the models developed by the Pisa group, and taking into account evolutionary effects. We compare these masses with those expected at the tip of the red giant branch (RGB) to derive the total mass lost by the stars.

Results. We find that a simple linear dependence on metallicity of this total mass lost describes quite well the median colours of HB stars. Assuming this mass loss law to be universal, we find that age is the main second parameter, determining many of the most relevant features related to HBs. In particular, it allows us to explain the Oosterhoff dichotomy as a consequence of the peculiar age-metallicity distribution of GCs in our Galaxy, although both Oosterhoff groups have GCs spanning a rather wide range of ages. However, at least an additional - third - parameter is clearly required. The most likely candidate is the He abundance, which might be different in GC stars belonging to the different stellar generations whose presence was previously derived from the $\mathrm{Na}-\mathrm{O}$ and $\mathrm{Mg}-\mathrm{Al}$ anticorrelations. Variations in the median He abundance allow us to explain the extremely blue HB of GCs like NGC 6254 (=M 10) and NGC 1904 (=M 79); such variations are found to be (weakly) correlated with the values of the $R$-parameter (that is the ratio of the number of stars on the HB and on the RGB). We also show that suitable He abundances allow deriving ages from the HB which are consistent with those obtained from the Main Sequence. Small corrections to these latter ages are then proposed. We find that a very tight age-metallicity relation (with a scatter below 4\%) can be obtained for GCs kinematically related to the disk and bulge, once these corrections are applied. Furthermore, star-to-star variations in the He content, combined with a small random term, explain very well the extension of the HB. There is a strong correlation between this extension and the interquartile of the Na-O anticorrelation, strongly supporting the hypothesis that the third parameter for GC HBs is He. Finally, there are strong indications that the main driver for these variations in the He-content within GCs is the total cluster mass. There are a few GCs exhibiting exceptional behaviours (including NGC 104=47 Tuc and in less measure NGC 5272=M 3); however, they can be perhaps accommodated in a scenario for the formation of GCs that relates their origin to cooling flows generated after very large episodes of star formation, as proposed by Carretta et al. (2009d).

Key words. globular clusters: general - stars: abundances

\section{Introduction}

Sandage \& Wallerstein (1960) noticed that the distribution with colour/temperature of stars on the horizontal branch (HB) of globular clusters (GCs) is roughly correlated with their metal content. A few years later, this observation was explained by the first successful models of HB stars describing the effect of metal content on the efficiency of H-shell burning in low mass stars where He is burning in the core (Faulkner 1966). However,

* Tables 1-8 and 10-12 are only available in electronic form at the CDS via anonymous ftp to cdsarc.u-strasbg.fr $(130.79 .128 .5)$ or via

http: //cdsweb.u-strasbg.fr/cgi-bin/qcat? J/A+A/517/A81 soon after this important theoretical achievement, van den Bergh (1967) and Sandage \& Wildey (1967) pointed out that the correlation between colour/temperature and metallicity had several exceptions, a difficulty that has become known as the second parameter problem. In the following forty years, a large number of tentative explanations for this discrepancy appeared in the literature, but no overall satisfactory scenario has yet been found. A proof of the large interest raised by this issue is that entering "globular cluster" and "second parameter" in the ADS data base $^{1}$ resulted in 231 abstracts (24 since 2006) with 6031 citations on a query made on April 15th, 2009. Of course, this search

\footnotetext{
${ }^{1}$ http://adsabs.harvard.edu/
} 
is probably incomplete, because there are many related issues, e.g., the Oosterhoff dichotomy in the mean periods of RR Lyrae in galactic GCs (Oosterhoff 1944; Sandage 1982), the UV upturn in the spectra of bulges and elliptical galaxies (Code 1969), the ages and the He abundances of GCs (Iben 1968), the mass loss law for low mass stars, which use different keywords. The second parameter problem is certainly one of the major open issues in our understanding of the evolution of normal stars. For reviews of this topic, we refer to Moehler (2001) and Catelan (2009a).

There are various reasons why the second parameter issue has been insofar so difficult to solve. The most important is that there is most likely more than a single second parameter. The colour of HB stars is very sensitive to several physical stellar quantities in the age and metallicity regime typical of GCs (see e.g., Rood 1973; Renzini 1977; Freeman \& Norris 1981; Fusi Pecci et al. 1993). Zinn (1980) and many authors after him convincingly showed that younger ages might explain the red colours of the HB of several of the outer halo GCs (see Dotter et al. 2008, for a similar line of thought). However, progress in the determination of the (relative) ages of GCs (e.g., Stetson et al. 1996; Rosenberg et al. 1999; De Angeli et al. 2005; Marín-Franch et al. 2009) demonstrated that this cannot be the only second parameter. The same result had previously been obtained even more directly from the broad distribution in colours of HB stars within some individual GCs (see e.g., Ferraro et al. 1990).

Since a change in the mass of stars on the HB may well cause even large variations in their colours (Rood 1973), a special mass-loss law has become a popular explanation (see Dotter 2008, for an example of this approach). Unfortunately, the physics of mass loss is very poorly known at present (see e.g., Willson 2000; Mészáros et al. 2009; Dupree et al. 2009). Many different mechanisms may affect mass loss (see e.g., Rich et al. 1997; Green et al. 1997; Soker \& Harpaz 2000) and empirical evidence is inadequate for fully constraining them (see e.g., Peterson 1982; Origlia et al. 2007, 2008). Given these limitations, it is not yet possible to build an ab-initio model for the estimation of mass loss from GC stars. Hence we prefer to carefully restrict our assumptions: we looked for solutions with a mass loss law based on as few simple parameters as possible, for which we may obtain constraints from independent observations.

Additional second parameters considered included He abundances, the ratio of CNO to Fe abundances, stellar rotation or binarity (see e.g., Freeman \& Norris 1981), and cluster concentration (Fusi Pecci et al. 1993). However, all of these explanations were found to be unsatisfactory overall. In the most successful cases, they might explain some groups of stars with anomalous colours on the HB (e.g., most field O-B subdwarfs are binaries, see e.g., Maxted et al. 2001; Napiwotzki et al. 2004; Han et al. 2003; however most of these in GCs seem to be single stars, see e.g., Moni Bidin et al. 2006). In the least successful cases, they are inconsistent with the data (CNO abundances: see the case of the second parameter pair NGC 362 and NCC 288: Shetrone $\&$ Keane 2000; and Catelan et al. 2001). The situations for rotation and $\mathrm{He}$ abundances are more complicated. For rotation, after the initial enthusiasm triggered by the pioneering observations of Peterson (1982), the problem was found to be less straightforward. More extensive data sets (Deliyannis et al. 1989; Behr et al. 2000a,b; Behr 2003; Recio-Blanco et al. 2002) revealed an intricate pattern, so that it seems difficult to use direct observations of rotation along the HB to confirm its rôle in the second parameter issue. While these observations do not rule out the possibility that rotation is indeed important, we cannot avoid concluding that the evidence for and against remains poor (see e.g., Sweigart 2002). We discuss the case of He abundances in subsequent sections.

Over the years, an enormous wealth of observational data has been collected, not only in terms of the distribution of stars along the $\mathrm{HB}$ with colour, but also the chemical composition, the period distribution of RR Lyrae, and other properties of GCs. Several authors (see e.g., Fusi Pecci et al. 1993; Catelan et al. 2001; Recio-Blanco et al. 2006; Carretta et al. 2007) pointed out the existence of correlations between global cluster parameters (such as luminosity, concentration, or Galactic orbit) and phenomena related to the second parameter. However, the mechanism connecting these global properties to the evolution of individual stars remained elusive until a few years ago.

A revised approach to the problem of the second parameter can now be developed. It is based on what was initially considered to be a separate characteristic of GCs, that is the abundance anomalies observed for GCs in the light elements $\mathrm{CNO}$, $\mathrm{Na}, \mathrm{Mg}$, and $\mathrm{Al}$ (see Kraft 1994; and Gratton et al. 2004, for reviews of this topic). Early suggestions that there might be a correlation between these two sets of observations were made by Norris (1981), and more recently by Kraft (1994) and Catelan \& de Freitas Pacheco (1995) and several other authors after them. However, the exact mechanism linking the two phenomena remained unclear. The two innovative steps taken subsequently were:

- the recognition that typical GCs host at least two generations of stars: this is required to explain the abundance anomalies observed for main sequence (MS) stars by Gratton et al. (2001) and Cohen et al. (2002). These observations contradicted the paradigm of GCs as single stellar populations, and opened a new view on GC formation and evolution that we are now only beginning to explore (see Gratton et al. 2004, for early results);

- the understanding by D'Antona et al. (2002) that (large) variations in the abundance of $\mathrm{He}$, which are expected to be correlated with the variations in $\mathrm{Na}$ and $\mathrm{O}$ and other elements, might result in large differences in the turn-off (TO) masses of stars of similar age: this is because He-rich stars evolve faster than He-poor ones and thus, at a given age, He-rich stars at TO are less massive ${ }^{2}$. Therefore, similar mass losses along the red giant branch (RGB) would lead to HB stars of very different masses, and hence colours. Not all authors estimated variations in the He abundances as large as those proposed by D'Antona et al. (2002; see for instance Marcolini et al. 2009). We note however that these small spreads in He abundance (which are usually justified on the basis of chemical evolution models) have difficulties in reproducing the observed spread in masses along the HB and the splitting of the MS of NGC 2808 (Lee et al. 2005; Piotto et al. 2007).

That a combination of age and He differences may explain the second parameter is very attractive for several reasons: (i) the large variations in $\mathrm{He}$ and the $\mathrm{Na}-\mathrm{O}$ anticorrelation are explained by the presence of different generations of stars in GCs, and it is then easy to link them to general cluster properties, such as their

\footnotetext{
2 As pointed out by the referee, the knowledge that He abundance variations lead to variations in turn-off masses is much older (see Iben \& Rood 1970). However, D'Antona et al. were the first to relate variations in $\mathrm{Na}, \mathrm{O}$, and other light elements to variations in He abundances, and then TO masses. This created the link between abundance anomalies on the RGB and the second parameter issue.
} 
mass or location in the Galaxy, which seem to play a rôle in the second parameter issue; (ii) these different stellar generations may well be used to explain discontinuous and often discrete distributions of stars along the HB, such as that observed e.g., in NGC 2808 (D'Antona et al. 2005); (iii) very accurate photometric data detect multiple main sequences in some GCs (Bedin et al. 2004; Piotto et al. 2007) that can only be explained by assuming large variations in the He content (Norris et al. 2004; Piotto et al. 2005; Milone et al. 2010).

While these observations are extremely interesting, they are rather limited in number: the data discussed insofar only concern a handful of massive GCs. A more comprehensive study of a large set of GCs, analysed in a homogeneous way, was lacking until recently. Such an analysis is now possible, thanks to the large databases of colour magnitude diagrams (CMD) and accurate ages provided by ground (Rosenberg et al. 2000a,b) and space observations (e.g., Piotto et al. 2002), and the extensive data on the $\mathrm{Na}-\mathrm{O}$ anticorrelation from our FLAMES survey (Carretta et al. 2009a,b, and references therein), complemented by literature data. In this paper, we present an exploration of these unprecedented databases. In the first part of the paper, we consider the evidence provided by extensive photometric datasets, from both ground-based and HST observations for a sample of almost a hundred GCs, deriving the properties of HBs as defined by their median values and extension, and examining their correlations with metallicity and age. This analysis produces a simple mass-loss law, that explains the median colours of HB stars. However, as found by several authors before, an additional parameter is needed to explain the HB colours of GCs with an extreme blue HB (BHB) and the spread of colours in many other cases. In the second part of the paper, we consider variations in the He content as a possible explanation of these discrepancies, we derive the $\mathrm{He}$ abundance variations required explaining the observed properties of the HBs, and discuss the implications for MS photometry. In the third part of the paper, we search for additional evidence that $\mathrm{He}$ is indeed the additional parameter required to explain the HB morphology. In particular, we explore the correlations with other chemical anomalies, namely the $\mathrm{Na}-\mathrm{O}$ anticorrelation. For this purpose, we consider a smaller but still quite large sample of $24 \mathrm{GCs}$, including classical second parameter cases (NGC 288 and NGC 362; NGC 5272 and NGC 6205), a list of blue HB clusters (such as NGC 1904 or NGC 6752), and the very extended HB cases (such as NGC 2808, NGC 6388 and NGC 6441). The aim of our discussion is to convince the reader that a combination of age and He abundance variations, the latter being related to multiple generations of stars within each GC, is a promising scenario to clarify most of the so far unexplained characteristics of the second parameter issue. We emphasise that we do consider that additional effects (e.g., binarity) may affect the colour of stars along the $\mathrm{HB}$, but the rôles played by the age and He abundance variations are probably dominant.

\section{Median and extreme colours of stars along the HB}

Several authors have suggested that age is the (main) second parameter determining the colour of HB stars (e.g., Zinn 1985; Demarque et al. 1989; Lee et al. 1994). In this section, we present a revised evaluation of this issue, performing an extensive comparison between observations and models. We exploit the large databases of globular cluster photometry available on the web and the latest age and metallicity estimates.

\subsection{Observational data}

The first step of our procedure was to derive the median and range of colours and magnitudes (hereinafter the range including $90 \%$ of the stars) along the HB. We derived these quantities from the databases of ground-based and HST (snapshot) observations presented by Rosenberg et al. (2000a,b) and Piotto et al. (2002), respectively. These databases were selected because they are publicly available and include a large number of clusters analysed with very homogeneous methods. The HST-ACS survey (Sarajedini et al. 2007) is providing new, very high quality data, which will largely supersede those older catalogues; unfortunately, this data set is not publicly available yet ${ }^{3}$. However, as we will see, the older photometry is of excellent quality, with only a few caveats. The ground database includes $V$ and $I$ CMDs and photometric tables for $52 \mathrm{GCs}$, from which we dropped E3, which is too scarcely populated for the present purposes, and $\omega$ Cen, whose spread in metal abundance makes the interpretation of data far more complicated than for the remaining clusters. The HST database contains $F 439 W$ and F555W CMDs and photometric tables for $71 \mathrm{GCs}$. In the following, we considered $B$ and $V$ colours obtained from the HST photometry through the transformations given by Piotto et al. (2002). There are 23 clusters in common between the two samples, so data are available for a total of $98 \mathrm{GCs}$.

Table 1 gives basic parameters for the clusters. Metallicities $[\mathrm{Fe} / \mathrm{H}]$ are from our re-analysis of GC metallicity (Carretta et al. 2009c; note that we adopted a metallicity of $[\mathrm{Fe} / \mathrm{H}]=-1.35$ for NGC 6256, from Stephens \& Frogel 2004), while apparent distance moduli $(m-M)_{V}$, reddenings $E(B-V)$, absolute magnitudes $M_{\mathrm{V}}$, and HBR are from the Harris catalogue (1996). Relative ages are from the compilation of Carretta et al. (2009d), and are mainly from Marín-Franch et al. (2009) and De Angeli et al. (2005, this last modified to be on the same scale), corrected to have values consistent with the metallicities by Carretta et al. (2009c). Masses at both the turn-off $\left(M_{\mathrm{TO}}\right)$ and the tip of the RGB $\left(M_{\mathrm{RGB}}\right)$ were computed as explained in Sect. 2.4. IQR values are the interquartile of the $\mathrm{Na}-\mathrm{O}$, and $\mathrm{Mg}$-Al (anti-)correlations and come from Carretta et al. (2009b), with a few additional data from literature (Shetrone \& Keane 2000, for NGC 362; Sneden et al. 2004; and Cohen \& Melendez 2005, for NGC 5272 and NGC 6205; Marino et al. 2008, 2009, for NGC 6121 and NGC 6656, respectively; and Yong et al. 2005, for NGC 6752). Finally, $\log T_{\text {eff }}^{\max }(\mathrm{HB})$ is the maximum temperature of $\mathrm{HB}$ stars, from Recio-Blanco et al. (2006), complemented by data for a few clusters evaluated by Carretta et al. (2009d). For each cluster,

\footnotetext{
3 An analysis of the ages of GCs and median colours of the HB based on these ACS data has been published after this paper was written (Dotter et al. 2010). A complete comparison with our results would require a long section. However, we note that there are very tight correlations between the $V-I$ colours from ACS data, and the $B-V$ and $V-I$ ones of this paper. The ages by Dotter et al. also agree fairly well, at least in a statistical sense, with those considered in this paper. Not surprisingly, there is also agreement at least in the first major conclusion: age is the main second parameter. However, Dotter et al. suggest cluster concentration as third parameter, while we propose absolute magnitudes, on turn related to the He content. We note that Dotter et al. did not examine the extension of the HB, limiting their study to the median colours. We argue that it is not easy to discuss this third parameter using this approach. Furthermore, there is some correlation between luminosity and concentration for GCs, which may justify their result. However, our analysis of the extension of the HB shows that this is much more strongly correlated with the cluster absolute magnitudes, rather than their concentration.
} 
Table 1. Basic parameters for globular clusters considered in this paper ( $M_{\mathrm{TO}}$ and $M_{\mathrm{RGB}}$ are in solar masses).

\begin{tabular}{|c|c|c|c|c|c|c|c|c|c|c|c|c|}
\hline Cluster & Other & $\begin{array}{c}{[\mathrm{Fe} / \mathrm{H}]} \\
\operatorname{dex}\end{array}$ & $\begin{array}{c}(m-M)_{V} \\
\text { mag }\end{array}$ & $\begin{array}{c}E(B-V) \\
\text { mag }\end{array}$ & $\begin{array}{c}M_{\mathrm{V}} \\
\text { mag }\end{array}$ & HBR & Age & $\begin{array}{c}M_{\mathrm{TO}} \\
M_{\odot}\end{array}$ & $\begin{array}{c}M_{\mathrm{RGB}} \\
M_{\odot}\end{array}$ & $\begin{array}{l}\mathrm{IQR}_{[\mathrm{Na} / \mathrm{O}]} \\
\quad \operatorname{dex}\end{array}$ & $\begin{array}{l}\mathrm{IQR}_{[\mathrm{Al} / \mathrm{Mg}]} \\
\quad \operatorname{dex}\end{array}$ & $\log T_{\mathrm{eff}}^{\max }(\mathrm{HB})$ \\
\hline NGC 104 & 47 Tuc & -0.76 & 13.37 & 0.04 & -9.42 & -0.99 & 0.95 & 0.862 & 0.909 & 0.472 & 0.091 & 3.756 \\
\hline NGC 288 & & -1.32 & 14.83 & 0.03 & -6.74 & 0.98 & 0.90 & 0.827 & 0.869 & 0.776 & 0.059 & 4.221 \\
\hline NGC 362 & & -1.30 & 14.81 & 0.03 & -8.41 & -0.87 & 0.80 & 0.858 & 0.900 & 0.670 & & 4.079 \\
\hline IC 1257 & & -1.73 & 19.25 & 0.73 & -6.15 & -0.71 & & & & & & \\
\hline NGC 1261 & & -1.27 & 16.10 & 0.01 & -7.81 & 1.00 & 0.79 & 0.847 & 0.887 & & & 4.079 \\
\hline
\end{tabular}

Notes. The complete table is available at the CDS.

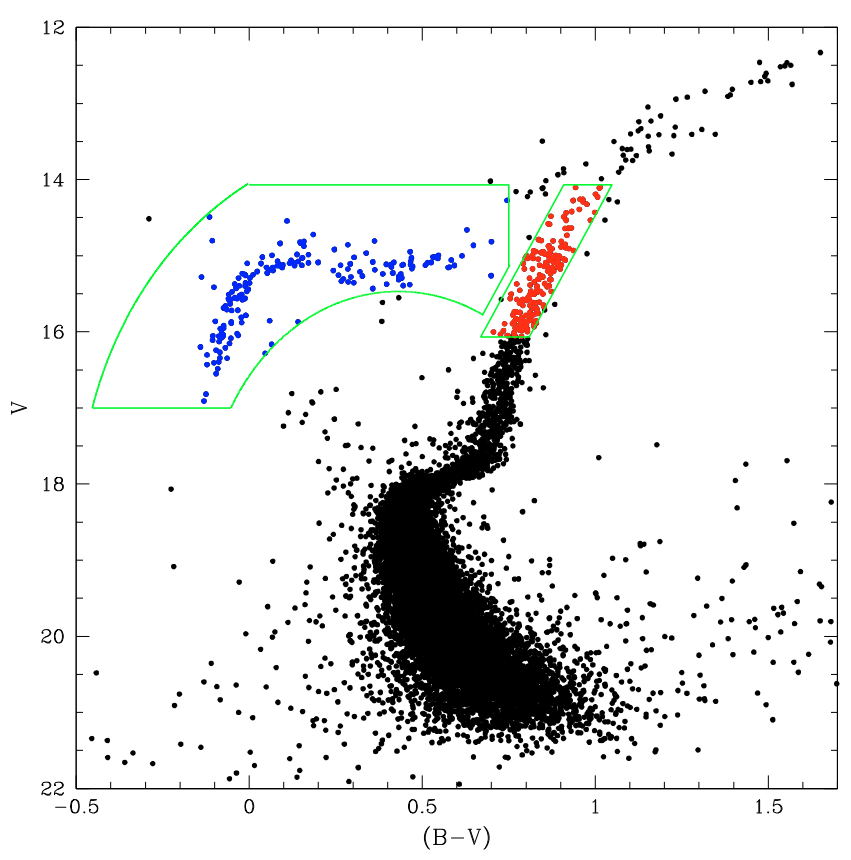

Fig. 1. Observed CMD for NGC 5904. RGB and HB stars are marked with red and blue points, respectively, while the green boxes represent the selection region of each component.

we first identified the region covered by the $\mathrm{HB}$, as well as that occupied by RGB stars that are within \pm 1 mag of the mean magnitude of the $\mathrm{HB} V(\mathrm{HB})$, taken from the on line version of the Harris catalogue of GCs (Harris 1996). Figure 1 shows an example of the selection of HB and RGB stars.

Completeness of the photometry can be an issue in a statistical study such as the present one. HST-based observations are generally complete to magnitude $V \sim 21.5$ (with some exceptions on both the brighter and fainter side), which is much fainter than the HB level at the RR Lyrae colour in the vast majority of GCs of this sample. We tested the completeness of the HB in the HST photometry by checking whether the number of HB stars is not unexpectedly small compared to the number of RGB stars that are within \pm 1 mag of $V(\mathrm{HB})$ (see Fig. 2). On average, this ratio is $0.934 \pm 0.020$ for GCs with HST data. The only cluster for which there is a clear deficiency of observed HB stars (a value smaller than the average one by more than twice the standard deviation, as estimated by Poisson statistics) is the farthest one (NGC 2419: Harris et al. 1996), for which this ratio is $0.56 \pm 0.09$. The next smallest value is for NGC 6624 , for which the value is $0.70 \pm 0.14$, which is only 1.6 standard deviation below the average value. However, the ground-based observations are shallower, with typical limiting magnitudes in the range 19-20. For this reason, extreme HB stars are sometimes missing from these data; we then generally give preference to the HST

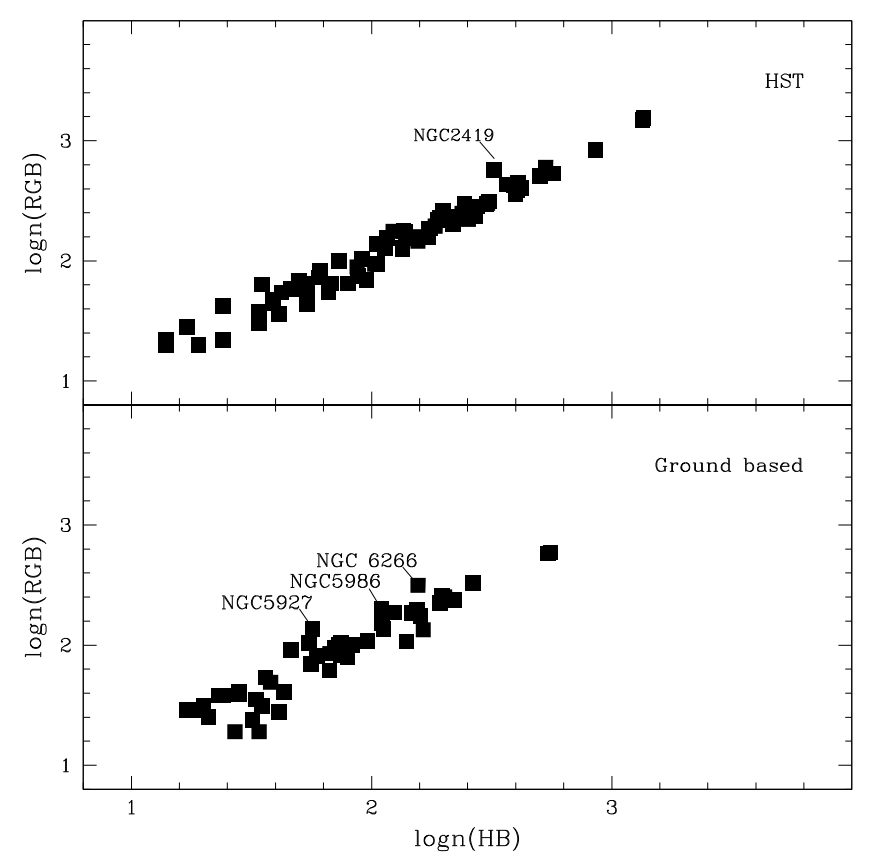

Fig. 2. Comparison between the number of $\mathrm{HB}$ and RGB stars that are within \pm 1 mag of the mean magnitude of the HB $V(\mathrm{HB})$. Upper and lower panels are for HST and ground-based data. Labels indicate the points relative to those GCs for which star counts are not reliable, using this diagnostics.

snapshot data in our discussion. However, the ground-based data provide very useful information about a number of nearby GCs missing in the Piotto et al. (2002) database ${ }^{4}$.

Particular care was devoted to the separation between the HB and the RGB, which is difficult for very metal-rich GCs when differential reddening is large. We also tried to minimize the impact of contamination by field stars, which may significantly affect the determination of the colour extremes, but only marginally affect the determination of median colours and magnitudes, which are robust estimators. Unfortunately, while very extensive and homogeneous, the databases that we used include neither comparison fields allowing a (statistical) subtraction of field contaminants, nor a full membership study. Our procedure was to first estimate those field stars expected to fall within the region of the CMD that we identified with the HB (Col. 3 of Table 2) and the RGB, based on the galactic model

\footnotetext{
4 The databases we considered actually also include entries for variable stars (on the HB mainly RR Lyrae). These entries are very inaccurate, since usually data at very few epochs are available, and photometry in different bands is not based on data acquired simultaneously. However, while inaccurate, these data still have some statistical meaning; for this reason, they are considered throughout our analysis.
} 
R. G. Gratton et al.: The second and third parameters of the horizontal branch
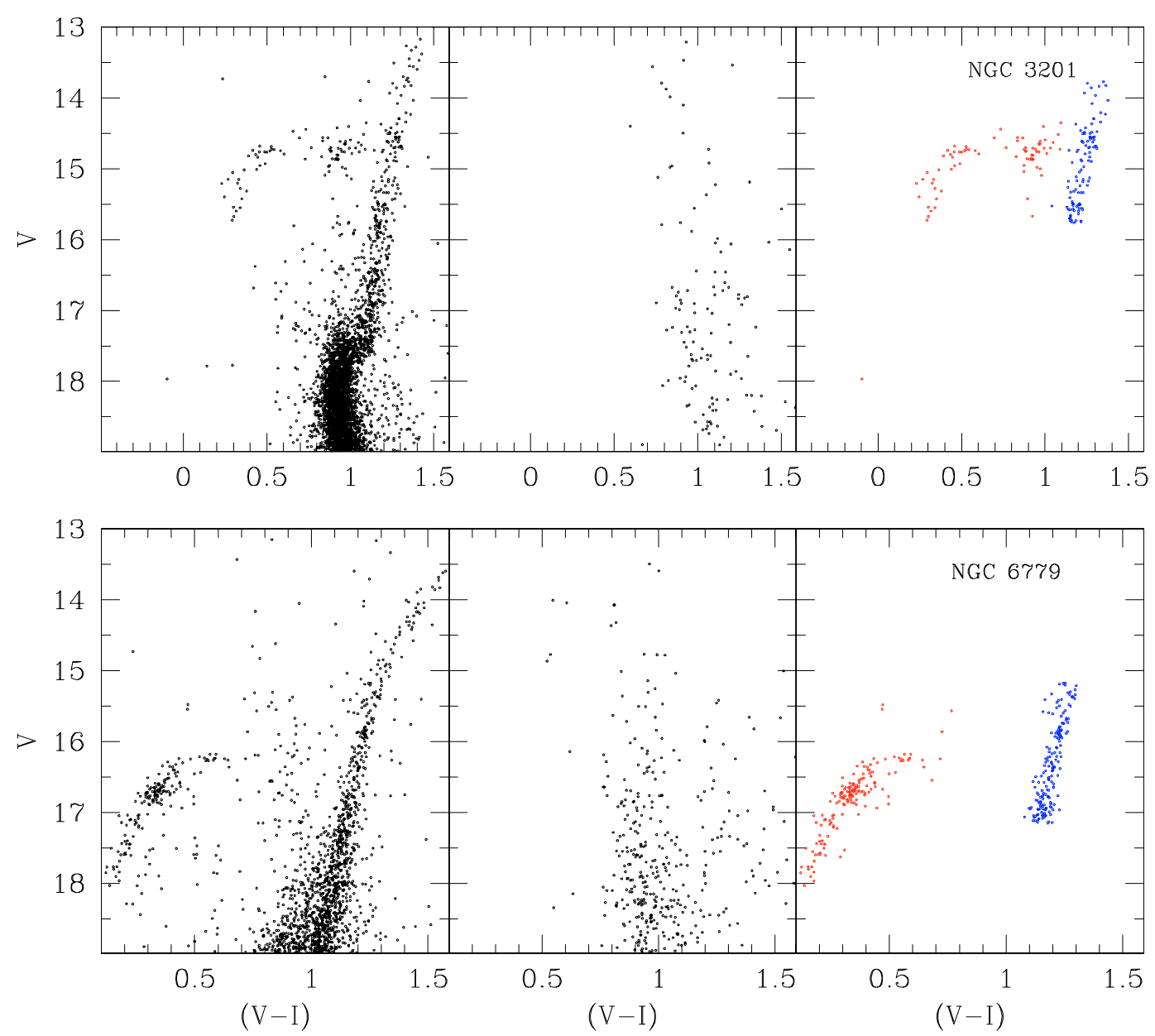

Fig. 3. Two examples of the application of the statistical field subtraction used in this paper (top: NGC 3201; bottom: NGC 6779); both cases are based on ground-based photometry. The left panels give the original photometry; the central panels give the CMD of field stars appropriate for the direction and reddening of the GCs, from the TRILEGAL model of the Milky Way (Girardi et al. 2005; Vanhollebeke et al. 2009); the right panels are the field-subtracted HB (red points) and RGB (blue points). The RGB stars are only those within \pm 1 mag of the HB level, according to Harris (1996).

TRILEGAL (Girardi et al. 2005; Vanhollebeke et al. 2009). We then subtracted from the CMD the star closest to each field star (weighting differences in colours five times more than differences in magnitudes); the numbers of HB and RGB stars given in this paper are those after this subtraction. This procedure introduces some uncertainty in our results, in particular in cases of strongly contaminated fields. However, eye inspection of the colour-magnitude diagrams obtained using this procedure shows that it worked satisfactorily. Figure 3 shows a couple of examples of this decontamination.

We typically identified a few hundred HB stars, and a similar number of RGB stars. However, in a few cases we identified only a few tens of stars belonging to the two sequences. In these cases, larger uncertainties are associated with the extreme values, while the median is still quite robust (see however Sect. 6).

We fitted each observed HB with a polynomial (using the colour as an independent variable), whose degree varied (between 1 and 4) from cluster-to-cluster, depending on the extension of the HB. For each star, we then replaced the observed colour and magnitude with that corresponding to the closest position along the polynomial. The metrics that we considered weight differences in colours five times more than those in magnitudes. This procedure reduces the impact of outliers. We then ordered the stars in terms of either increasing colour or magnitude, and determined the median as well as the colours and magnitudes that include the central $90 \%$ of the distribution.

In the case of the RGB, we determined the average colour of the sequence at the magnitude of the HB by fitting a straight line through the points corresponding to the RGB-selected stars; in this case, we used the magnitude as an independent variable.

Values of observed colours and magnitudes are given in Tables 2 and 3 for HST and ground-based data, respectively. Error bars are only those from statistics. They do not include systematic errors, which are due to incompleteness at faint magnitudes (most relevant for ground-based observations), incorrect separation of the different sequences (sometimes possible for most metal-rich clusters with differential reddening), or uncertainties in decontamination by field stars.

\subsection{From observed to intrinsic colours}

Observed colours and magnitudes differ from intrinsic ones for mainly three reasons: (i) errors in photometric calibration and transformation to the standard system (colour and magnitude); (ii) distance to the stars; and (iii) interstellar absorption and reddening. To reduce the impact of these uncertainties, we used the difference between observed and predicted colours of the RGB at the HB level $\left((B-V)_{\mathrm{RGB}, 0}\right.$ and $(V-I)_{\mathrm{RGB}, 0}$ for HST and 
Table 2. Photometric data from the HST-snapshot (Piotto et al. 2002).

\begin{tabular}{|c|c|c|c|c|c|c|c|c|c|}
\hline Cluster & $n(\mathrm{HB})$ & $N_{\text {Field }}$ & $\begin{array}{c}F 555_{\mathrm{med}} \\
\mathrm{mag} \\
\end{array}$ & $\begin{array}{c}(F 439-F 555)_{\mathrm{med}} \\
\text { mag }\end{array}$ & $\begin{array}{c}F 555_{\min } \\
\mathrm{mag}\end{array}$ & $\begin{array}{c}(F 439-F 555)_{\min } \\
\text { mag }\end{array}$ & $\begin{array}{c}(F 439-F 555)_{\max } \\
\operatorname{mag}\end{array}$ & $n(\mathrm{RGB})$ & $\begin{array}{c}(F 439-F 555)_{\mathrm{RGB}} \\
\operatorname{mag}\end{array}$ \\
\hline NGC 104 & 363 & 0 & $14.062 \pm 0.002$ & $0.815 \pm 0.005$ & $14.047 \pm 0.003$ & $0.719 \pm 0.005$ & $0.896 \pm 0.081$ & 432 & $1.067 \pm 0.004$ \\
\hline NGC 362 & 274 & 0 & $15.517 \pm 0.003$ & $0.563 \pm 0.005$ & $15.900 \pm 0.168$ & $-0.003 \pm 0.036$ & $0.691 \pm 0.051$ & 282 & $0.865 \pm 0.004$ \\
\hline IC 1257 & 34 & 3 & $20.440 \pm 0.063$ & $0.722 \pm 0.032$ & $20.645 \pm 0.031$ & $0.599 \pm 0.024$ & $0.889 \pm 0.028$ & 38 & $1.522 \pm 0.013$ \\
\hline NGC 1261 & 135 & 0 & $16.821 \pm 0.009$ & $0.584 \pm 0.011$ & $17.324 \pm 0.129$ & $-0.047 \pm 0.038$ & $0.697 \pm 0.022$ & 124 & $0.849 \pm 0.006$ \\
\hline NGC 1851 & 307 & 0 & $16.205 \pm 0.017$ & $0.572 \pm 0.026$ & $16.908 \pm 0.094$ & $-0.057 \pm 0.010$ & $0.705 \pm 0.088$ & 310 & $0.919 \pm 0.004$ \\
\hline
\end{tabular}

Notes. The complete table is available at the CDS.

Table 3. Photometric data from ground-based data (Rosenberg et al. 2000a,b).

\begin{tabular}{lccccccccc}
\hline \hline Cluster & $n(\mathrm{HB})$ & $N_{\text {Field }}$ & $\begin{array}{c}V_{\text {med }} \\
\text { mag }\end{array}$ & $\begin{array}{c}(V-I)_{\text {med }} \\
\text { mag }\end{array}$ & $\begin{array}{c}V_{\min } \\
\operatorname{mag}\end{array}$ & $\begin{array}{c}(V-I)_{\min } \\
\text { mag }\end{array}$ & $\begin{array}{c}(V-I)_{\max } \\
\operatorname{mag}\end{array}$ & $\begin{array}{c}n(\mathrm{RGB}) \\
(V-I)_{\mathrm{RGB}} \\
\mathrm{mag}\end{array}$ \\
\hline NGC 104 & 192 & 0 & $13.970 \pm 0.002$ & $0.892 \pm 0.006$ & $13.996 \pm 0.003$ & $0.817 \pm 0.009$ & $0.955 \pm 0.004$ & 225 & $1.054 \pm 0.005$ \\
NGC 288 & 83 & 0 & $16.083 \pm 0.114$ & $0.022 \pm 0.022$ & $17.110 \pm 0.129$ & $-0.136 \pm 0.016$ & $0.228 \pm 0.531$ & 101 & $0.987 \pm 0.006$ \\
NGC 362 & 56 & 1 & $15.371 \pm 0.010$ & $0.779 \pm 0.015$ & $15.523 \pm 0.003$ & $0.135 \pm 0.020$ & $0.838 \pm 0.034$ & 69 & $0.973 \pm 0.007$ \\
NGC 1261 & 221 & 1 & $16.653 \pm 0.003$ & $0.736 \pm 0.005$ & $16.942 \pm 0.272$ & $0.026 \pm 0.095$ & $0.826 \pm 0.021$ & 239 & $0.926 \pm 0.004$ \\
NGC 1851 & 33 & 3 & $16.177 \pm 0.020$ & $0.800 \pm 0.037$ & $17.086 \pm 0.143$ & $-0.028 \pm 0.014$ & $0.855 \pm 0.009$ & 35 & $1.004 \pm 0.009$ \\
\hline
\end{tabular}

Notes. The complete table is available at the CDS.

ground-based observations, respectively) to estimate the offset in colours appropriate for each cluster, and then corrected the median and the extreme colours of the HB for these offsets. To a first approximation, errors in reddening and photometric calibration should cancel out when determining these quantities. However, $(B-V)_{\mathrm{RGB}, 0}$ and $(V-I)_{\mathrm{RGB}, 0}$ both depend on metallicity. To estimate this dependence, we fitted with polynomials the relations obtained for clusters with only moderate reddening $(E(B-V)<0.25)$, using reddening from Harris (1996) and metallicities from Carretta et al. (2009c). We obtained the relations

$(B-V)_{\mathrm{RGB}, 0}=1.235+0.4397[\mathrm{Fe} / \mathrm{H}]+0.0927[\mathrm{Fe} / \mathrm{H}]^{2}$,

and

$(V-I)_{\mathrm{RGB}, 0}=1.154+0.2066[\mathrm{Fe} / \mathrm{H}]+0.0400[\mathrm{Fe} / \mathrm{H}]^{2}$

for HST (33 clusters, rms $=0.026 \mathrm{mag}$ ) and ground-based data (29 clusters, $\mathrm{rms}=0.039 \mathrm{mag}$ ), respectively (see Fig. 4 ).

Inspection of the upper panel of Fig. 4 reveals that there is a systematic trend in which $(B-V)_{\mathrm{RGB}, 0}$ values for highly reddened metal-rich GCs are systematically below the calibration curve, when using the HST photometry. This discrepancy might have either of three causes (or a combination of them): (i) metallicity or (ii) reddening for these clusters are overestimated; or (iii) the colour transformation at very red colours $((B-V)>1.5)$ is incorrect, producing too red colours for these stars. Errors in reddening have no impact in our analysis, since the procedure we adopted should cancel their effect. The impacts of errors in the metallicity scale and colour transformations are more difficult to trace throughout the analysis. However, in general they would lead to a small underestimate of the masses of HB stars in these clusters; this would reduce the sensitivity to metallicity of the mass-loss law we derive later, but should have no other major impact on our analysis.

We estimated intrinsic colours by adding the offsets from the mean relations (for $(B-V)_{\mathrm{RGB}, 0}$ and $\left.(V-I)_{\mathrm{RGB}, 0}\right)$ to the observed colours. Median and extreme of the colours are plotted against metallicity in Figs. 5 and 6, for HST and ground-based data, respectively. Different symbols are used for clusters of different relative ages (see Carretta et al. 2009d). In particular, we

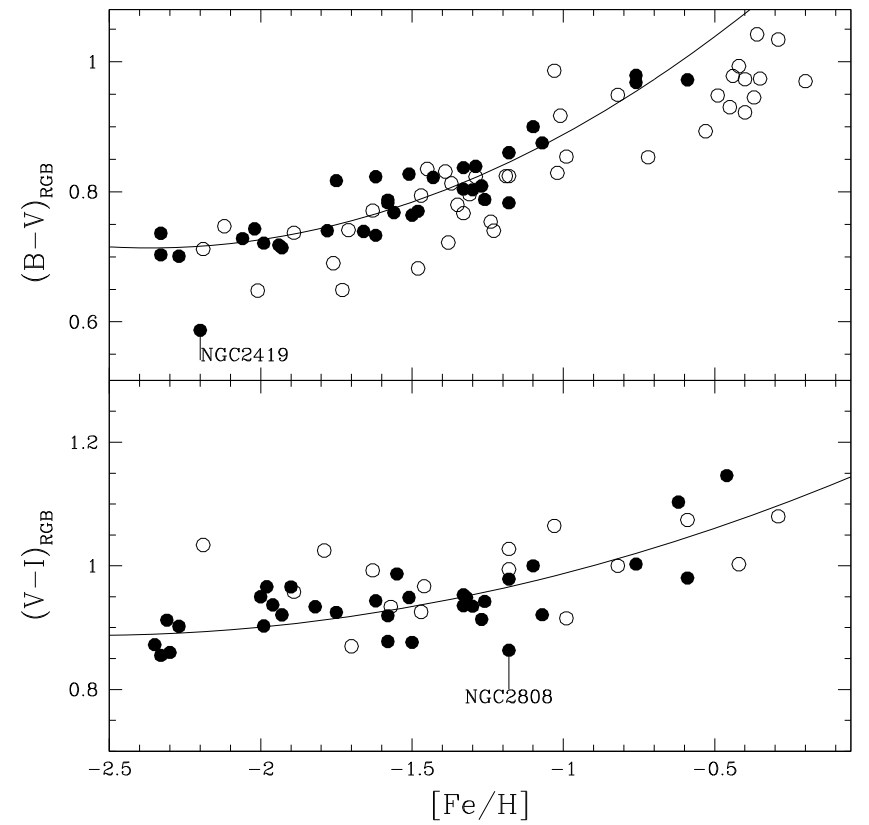

Fig. 4. Run of $(B-V)_{\mathrm{RGB}, 0}$ and $(V-I)_{\mathrm{RGB}, 0}$ with metallicity. Open and filled symbols represent clusters with $E(B-V)$ larger and smaller than 0.25 , respectively, while the solid curves show the relations given by Eqs. (1) and (2). A few points relative to interesting clusters are marked.

note that old clusters define a tight, unique (although quite complex) relation between metallicity and median colour of the $\mathrm{HB}$. This indicates that the median location of the HB is uniquely determined by age and metallicity. The relation between colours and masses of HB stars is however not linear, with the wellknown, very strong sensitivity of colours on metallicities for the metallicity range $-1.6<[\mathrm{Fe} / \mathrm{H}]<-1.1$. This is more clearly explained in the next subsection. Younger clusters typically have redder median colours than older ones at a given metallicity, as expected. Finally, clusters with no age determination roughly occupy the same region identified by the other clusters, but they seem to have a larger scatter. This is not unexpected, since 
R. G. Gratton et al.: The second and third parameters of the horizontal branch

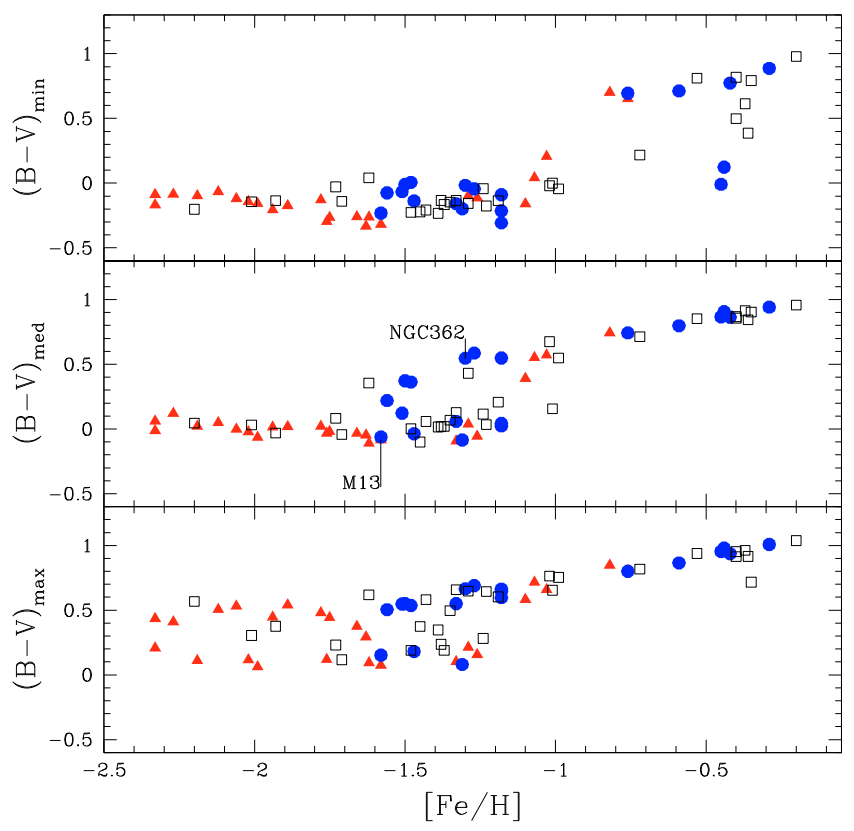

Fig. 5. Median and extreme colours as a function of metallicity for clusters observed with HST. Filled (red) triangles and (blue) dots indicate GCs older and younger than a relative age parameter of 0.92 , respectively; clusters for which an age estimate is not available are marked with (black) empty squares. Points representing a few interesting clusters are marked.

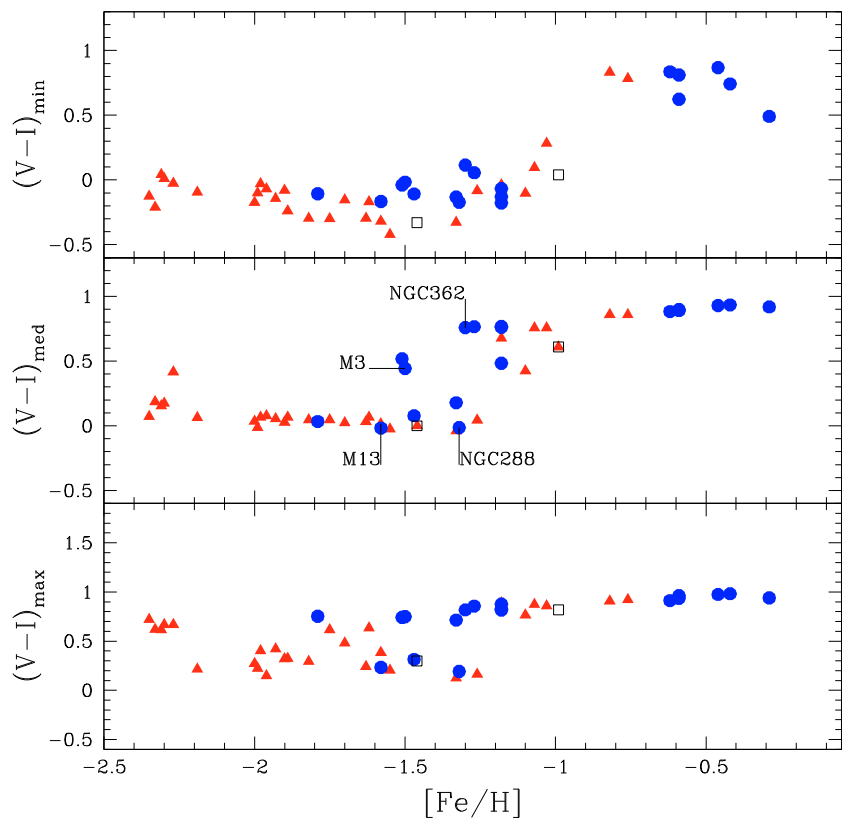

Fig. 6. Same as Fig. 5 but for ground-based sample.

data for these GCs are of poorer quality, and this is the reason why they lack an age estimate. We note also that much larger scatters are obtained for both minimum and maximum colours. We return to this point in the following.

In the case of magnitudes, we simply corrected the observed values for the apparent distance moduli given in Harris (1996).

We note that the plot of the median colour with metallicity is conceptually similar to a Lee-Zinn diagram (Lee \& Zinn 1990), since the median colours are very well correlated with the HBR parameter used in this diagram (see Fig. 7; for a definition of HBR, see Zinn 1985). The only exception to this close

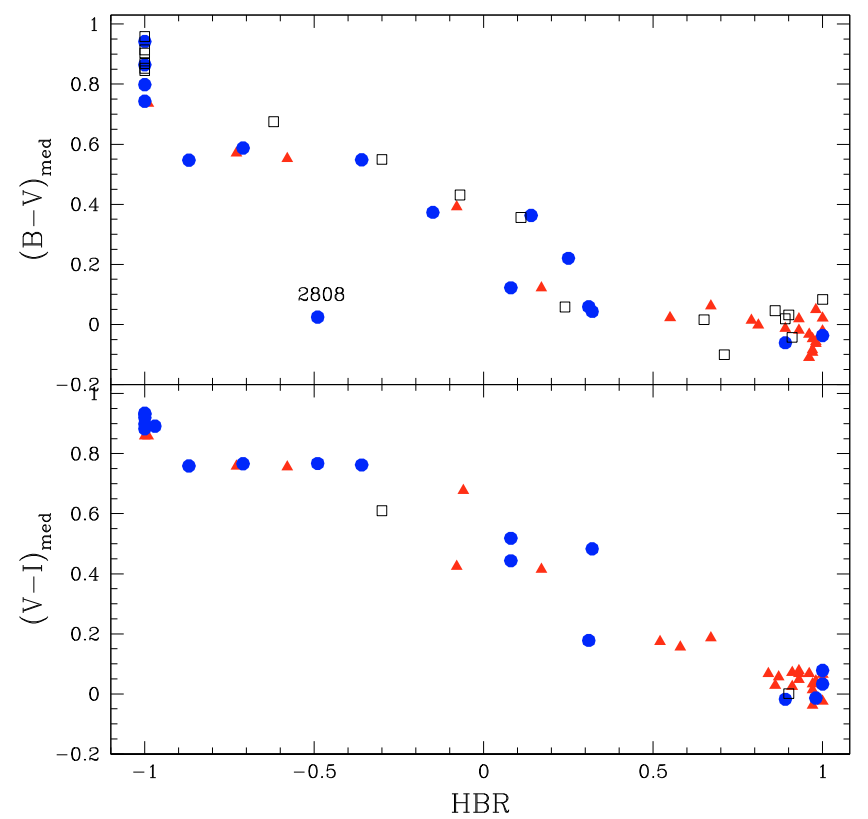

Fig. 7. Median colours versus HBR ratio for HST (upper panel) and ground-based (lower panel) clusters. Different symbols denote different ages (see Fig. 5).

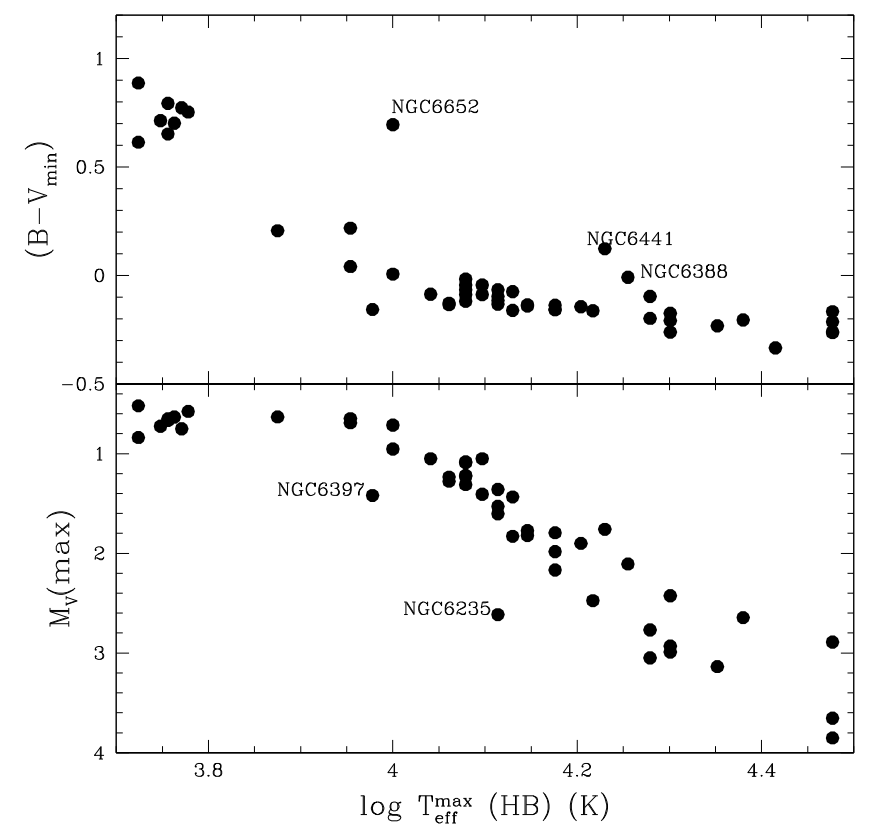

Fig. 8. Run of minimum colours and maximum magnitudes with the maximum temperatures $\log T_{\text {eff }}^{\max }(\mathrm{HB})$ retrieved by Recio-Blanco et al. (2006).

correlation is NGC 2808, when using HST (but not groundbased) data. This is due to the multi-modal distribution of stars along the $\mathrm{HB}$ of this cluster, and to the incompleteness of the ground-based photometry, which was used to derive the value of the HBR parameter. On the other hand, minimum colours and maximum absolute magnitudes along the HB are obviously well correlated with the maximum temperatures $\log T_{\mathrm{eff}}^{\max }(\mathrm{HB})$ determined by Recio-Blanco et al. (2006, see Fig. 8), which were derived from the same HST photometry. The few discrepant cases are either GCs with poor statistics or CMDs that are heavily contaminated with field stars, and for which different assumptions 
Table 4. Intrinsic HB photometric parameters for GCs with HST data.

\begin{tabular}{lcccccc}
\hline \hline Cluster & $\begin{array}{c}(B-V)_{0, \text { RGB }} \\
\text { mag }\end{array}$ & $\begin{array}{c}(B-V)_{\min } \\
\mathrm{mag}\end{array}$ & $\begin{array}{c}(B-V)_{\operatorname{med}} \\
\mathrm{mag}\end{array}$ & $\begin{array}{c}(B-V)_{\max } \\
\mathrm{mag}\end{array}$ & $\begin{array}{c}M_{V} \max \\
\mathrm{mag}\end{array}$ & $\begin{array}{c}M_{V} \operatorname{med} \\
\mathrm{mag}\end{array}$ \\
\hline NGC 104 & 0.968 & 0.652 & 0.736 & 0.807 & 0.651 & 0.665 \\
NGC 362 & 0.803 & -0.017 & 0.547 & 0.665 & 1.090 & 0.684 \\
IC 1257 & 0.649 & -0.029 & 0.083 & 0.231 & 1.371 & 1.164 \\
NGC 1261 & 0.809 & -0.044 & 0.587 & 0.690 & 1.227 & 0.697 \\
NGC 1851 & 0.860 & -0.096 & 0.534 & 0.656 & 1.442 & 0.712 \\
\hline
\end{tabular}

Notes. The complete table is available at the CDS.

Table 5. Intrinsic HB photometric parameters for GCs with ground-based data.

\begin{tabular}{lcccccc}
\hline \hline Cluster & $\begin{array}{c}(V-I)_{0, \mathrm{RGB}} \\
\mathrm{mag}\end{array}$ & $\begin{array}{c}(V-I)_{\min } \\
\mathrm{mag}\end{array}$ & $\begin{array}{c}(V-I)_{\operatorname{med}} \\
\mathrm{mag}\end{array}$ & $\begin{array}{c}(V-I)_{\max } \\
\mathrm{mag}\end{array}$ & $\begin{array}{c}M_{V} \max \\
\mathrm{mag}\end{array}$ & $\begin{array}{c}M_{V} \text { med } \\
\text { mag }\end{array}$ \\
\hline NGC 104 & 1.003 & 0.783 & 0.858 & 0.921 & 0.626 & 0.600 \\
NGC 288 & 0.949 & -0.172 & -0.014 & 0.192 & 2.280 & 1.253 \\
NGC 362 & 0.935 & 0.115 & 0.759 & 0.818 & 0.713 & 0.561 \\
NGC 1261 & 0.913 & 0.056 & 0.766 & 0.856 & 0.842 & 0.553 \\
NGC 1851 & 0.978 & -0.066 & 0.762 & 0.817 & 1.616 & 0.707 \\
\hline
\end{tabular}

Notes. The complete table is available at the CDS.

about the impact of contaminants were adopted here with respect to Recio-Blanco et al. (2006).

Values of intrinsic colours and magnitudes are given in Tables 4 and 5 for HST and ground-based data, respectively.

\subsection{From intrinsic colours to masses}

Our next step was to transform observed colours into masses of stars along the HB. The masses were obtained by comparing the observed colours and magnitudes with predictions from $\mathrm{HB}$ evolutionary models. For this purpose, we used the database of models computed by the Pisa evolutionary group (Castellani et al. 2003; Cariulo et al. 2004) ${ }^{5}$, which are particularly useful here since they provide a grid of HB evolutionary sequences for different masses and metallicities. We first derived the masses appropriate for ZAHB stars of the same colours/magnitudes of the observed loci on the HB, and then applied corrections appropriate to taking into account the evolution of the stars away from the ZAHB.

For the first step, we derived transformations from the evolutionary tracks given by

$$
\begin{aligned}
M(\mathrm{ZAHB})= & 0.5254-0.0650[\mathrm{Fe} / \mathrm{H}]-0.1181[\mathrm{Fe} / \mathrm{H}](B-V) \\
& +0.1425(B-V)-0.6560(B-V)^{2} \\
& +0.6277(B-V)^{3} \quad M / M_{\odot}
\end{aligned}
$$

and

$$
\begin{aligned}
M(\mathrm{ZAHB})= & 0.5271-0.0629[\mathrm{Fe} / \mathrm{H}]-0.0851[\mathrm{Fe} / \mathrm{H}](V-I) \\
& +0.1399(V-I)-0.6490(V-I)^{2} \\
& +0.6134(V-I)^{3} \quad M / M_{\odot}
\end{aligned}
$$

which are valid in the range $0.54<M_{\mathrm{ZAHB}}<0.72 \mathrm{M} / \mathrm{M}_{\odot}$ and $-2.5<[\mathrm{Fe} / \mathrm{H}]<-0.6$ (some extrapolation is required for the most metal-rich GCs). The similar equation for the $V$ magnitude is

$$
\begin{aligned}
M(\mathrm{ZAHB})= & 0.5544-0.07286[\mathrm{Fe} / \mathrm{H}]+0.005228[\mathrm{Fe} / \mathrm{H}] M_{V}^{2} \\
& -0.04555 M_{V}+0.010522 M_{V}^{2} \quad M / M_{\odot},
\end{aligned}
$$

5 http://astro.df.unipi.it/SAA/PEL/ZO.html which is valid for $M_{\mathrm{V}}>0.8$ and $-2.5<[\mathrm{Fe} / \mathrm{H}]<-0.6$.

The evolutionary corrections were obtained by deriving masses from median and extreme colours of synthetic HB diagrams, obtained by taking into account evolution, with respect to the values appropriate for the same set of stars when on the ZAHB. We obtained the correcting formulae

$$
\begin{aligned}
M_{\min }(\mathrm{ev})= & M_{\min }(\mathrm{ZAHB})-(0.0331-0.0140[\mathrm{Fe} / \mathrm{H}] \\
& \left.-0.099 M_{\min }(\mathrm{ZAHB})\right) \quad M / M_{\odot}, \\
M_{\text {med }}(\mathrm{ev})= & M_{\text {med }}(\mathrm{ZAHB})-(0.0246-0.0136[\mathrm{Fe} / \mathrm{H}] \\
& \left.-0.0733 M_{\text {med }}(\mathrm{ZAHB})\right) \quad M / M_{\odot},
\end{aligned}
$$

and

$$
\begin{aligned}
M_{\max }(\mathrm{ev})= & M_{\max }(\mathrm{ZAHB})-[0.0356+0.1972[\mathrm{Fe} / \mathrm{H}] \\
& +0.1020[\mathrm{Fe} / \mathrm{H}]^{2}-M_{\max }(\mathrm{ZAHB})(0.18936[\mathrm{Fe} / \mathrm{H}]) \\
& \left.+0.10727[\mathrm{Fe} / \mathrm{H}]^{2}\right] \quad M / M_{\odot},
\end{aligned}
$$

for minimum, median, and maximum mass, respectively.

In practice, we estimated masses from: (i) colours whenever these were redder than $(B-V)_{0}>0.1\left(\right.$ or $\left.(V-I)_{0}>0.2\right)$; (ii) magnitudes whenever colours were bluer than $(B-V)_{0}=(V-$ $I)_{0}<-0.1$; and (iii) by a weighted average of the values obtained from magnitudes and colours when they were in an intermediate range.

Not unexpectedly, there is a good correlation between the differences $M_{\max }-M_{\min }$ and $M_{\text {med }}-M_{\text {min }}$ (see Fig. 9), the regression line (for HST data) being $M_{\max }-M_{\min }=(1.184 \pm$ $0.097)\left(M_{\text {med }}-M_{\text {min }}\right)+\left(0.033 \pm 0.026 M_{\odot}\right.$ with a linear regression coefficient of $r=0.82$ (74 clusters). We note, however, that the estimate of the maximum mass is of much larger uncertainty than that of the median mass, because of the large evolutionary effects and the impact of smaller statistics. Furthermore, the median mass is on average much closer to the maximum than to the minimum mass, because of the difference between these two values is mainly due to a nearly constant offset of about $0.03-0.04 M_{\odot}$. In only a few extreme cases (e.g., NGC 2808) is the difference between $M_{\text {med }}$ and $M_{\max }$ sufficiently large to really affect the arguments made in the remainder of this paper. Hereinafter we thus do not refer to $M_{\max }$ in our discussion. 
R. G. Gratton et al.: The second and third parameters of the horizontal branch

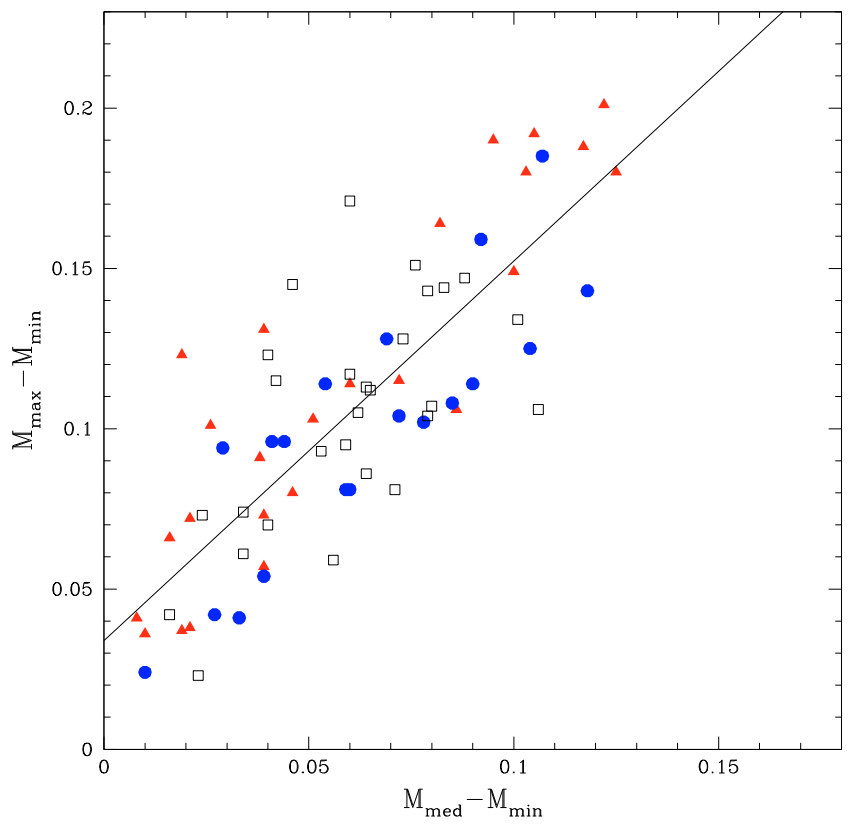

Fig. 9. Differences between maximum and minimum masses along the $\mathrm{HB}$, compared to differences between median and minimum masses, for clusters observed with HST. Filled (red) triangles and (blue) dots indicate GCs older and younger than a relative age parameter of 0.92 , respectively; clusters for which an age estimate is not available are marked with (black) empty squares.

We note that the lowest mass HB models considered here have a mass of $0.52 M_{\odot}$, the corresponding absolute magnitude predicted for these stars being $M_{\mathrm{V}}<4$. However, there are a few GCs for which the maximum absolute magnitude of HB stars is $M_{\mathrm{V}}>4$. In these cases, the masses given by our equations may be lower than the core masses at the He-flash for these models (Cassisi et al. 1998). While this might be indicative of either a different origin or a late He-flash for these stars (see e.g., D'Cruz et al. 1996), we note that in these cases our mass estimates are inferred from an extrapolation outside the range covered by the stellar models, and may therefore have a larger uncertainty. The value of the minimum mass along the HB for these GCs is certainly small, although probably not as small as indicated by our formulae.

Statistical errors in the mass estimates can be obtained by combining statistical errors in the colours with the errors in the metallicities. Errors in colours cause an uncertainty of about $0.007 M_{\odot}$ in the masses, while masses change by $-0.005 M_{\odot}$ if metallicity is increased by $+0.1 \mathrm{dex}$ in $[\mathrm{Fe} / \mathrm{H}]$.

The comparison between HST and ground-based results is as follows:

- minimum mass: ground-HST $=0.025 \pm 0.005 M_{\odot}$ (rms $0.024 M_{\odot}, 27$ clusters), where the scatter is mainly caused by GCs (NGC 2808, NGC 5986, and NGC $6266=$ M 62) with $(m-M)_{\mathrm{V}}>15.5$ and with BHB tails that are fainter than the limiting magnitude of the ground-based photometry. When these three clusters are eliminated, the offset is $0.019 \pm 0.003 M_{\odot}\left(\mathrm{rms} 0.016 M_{\odot}, 24\right.$ clusters $)$;

- median mass: ground-HST $=0.019 \pm 0.003 M_{\odot}(\mathrm{rms}=$ $0.019 M_{\odot}, 28$ clusters). The only clear outlier is NGC 2808. The offset is $0.016 \pm 0.003 M_{\odot}\left(\mathrm{rms}=0.015 M_{\odot}, 27\right.$ clusters) if this cluster is eliminated. This confirms that the median mass is far less sensitive to outliers and photometric errors than the minimum mass. The case of NGC 2808 is very
Table 6. Masses on HB for GCs with HST data.

\begin{tabular}{lccccr}
\hline \hline Cluster & $M_{\min }$ & $M_{\operatorname{med}}$ & $M_{\max }$ & $\Delta M_{\max }$ & $\Delta M_{\operatorname{med}}$ \\
& $M_{\odot}$ & $M_{\odot}$ & $M_{\odot}$ & $M_{\odot}$ & $M_{\odot}$ \\
\hline NGC 104 & $0.629 \pm 0.002$ & $0.648 \pm 0.001$ & 0.666 & 0.286 & 0.268 \\
NGC 362 & $0.602 \pm 0.002$ & $0.680 \pm 0.011$ & 0.704 & 0.297 & 0.219 \\
IC 1257 & $0.615 \pm 0.011$ & $0.655 \pm 0.001$ & 0.685 & & \\
NGC 1261 & $0.593 \pm 0.001$ & $0.683 \pm 0.007$ & 0.707 & 0.311 & 0.222 \\
NGC 1851 & $0.579 \pm 0.003$ & $0.664 \pm 0.005$ & 0.687 & 0.328 & 0.243 \\
\hline
\end{tabular}

Notes. The complete table is available at the CDS.

Table 7. Masses on HB for GCs with ground-based data.

\begin{tabular}{lccccc}
\hline \hline Cluster & $M_{\min }$ & $M_{\text {med }}$ & $M_{\max }$ & $\Delta M_{\max }$ & $\Delta M_{\text {med }}$ \\
& $M_{\odot}$ & $M_{\odot}$ & $M_{\odot}$ & $M_{\odot}$ & $M_{\odot}$ \\
\hline NGC 104 & $0.650 \pm 0.003$ & $0.674 \pm 0.003$ & 0.691 & 0.265 & 0.242 \\
NGC 288 & $0.570 \pm 0.001$ & $0.603 \pm 0.004$ & 0.645 & 0.302 & 0.269 \\
NGC 362 & $0.638 \pm 0.004$ & $0.702 \pm 0.006$ & 0.717 & 0.262 & 0.197 \\
NGC 1261 & $0.625 \pm 0.004$ & $0.701 \pm 0.005$ & 0.730 & 0.279 & 0.204 \\
NGC 1851 & $0.586 \pm 0.003$ & $0.688 \pm 0.009$ & 0.702 & 0.320 & 0.219 \\
\hline
\end{tabular}

Notes. The complete table is available at the CDS.

peculiar, because the distribution along the HB is very discontinuous and clumpy. If the extreme blue tail were neglected (as in the case of the ground based photometry), the median would jump from the blue $\mathrm{HB}$ to the red $\mathrm{HB}$, with a change in mass of about $0.04 M_{\odot}$.

Figure 10 illustrates the run of median and minimum masses with metallicity $[\mathrm{Fe} / \mathrm{H}]$, from HST and ground-based data, respectively. As for colours, we indicate with different symbols clusters of different relative ages. If we consider only the old clusters, the plots with median masses show a quite simple (although not linear) relation with metallicity, with a very small scatter about the mean line. Younger clusters scatter above the relation defined by the old ones, as expected. Again, clusters with no age estimates occupy a similar locus, but with a considerable scatter.

As noted in the case of colours, when we also consider minimum masses the scatter is much larger than obtained for the median. We underline that in spite of some uncertainties in our mass derivations this scatter is real, corresponding to a large variation in the appearance of the HBs: we note for instance the low minimum masses that are obtained for the GCs with very extended blue tails, such as NGC 2808 or NGC 7078 . We return to this point in Sect. 6.

Values for the minimum, median, and maximum masses obtained following the previous procedure are given in Tables 6 and 7, for HST and ground-based data, respectively.

\subsection{Mass-loss law}

The tight relation between median HB mass and metallicity for old GCs obtained in the previous subsection suggests that the median location of stars along the HB of a GC can be determined using a combination of metallicity, age, and a relatively simple and uniform mass-loss law. To show that this is indeed the case, we first estimated the original masses of stars currently on the HB. To determine this value, we first consider masses at TO $\left(M_{\mathrm{TO}}\right)$, which can be obtained from their age, chemical composition, and He content, by using appropriate models. In practice, 


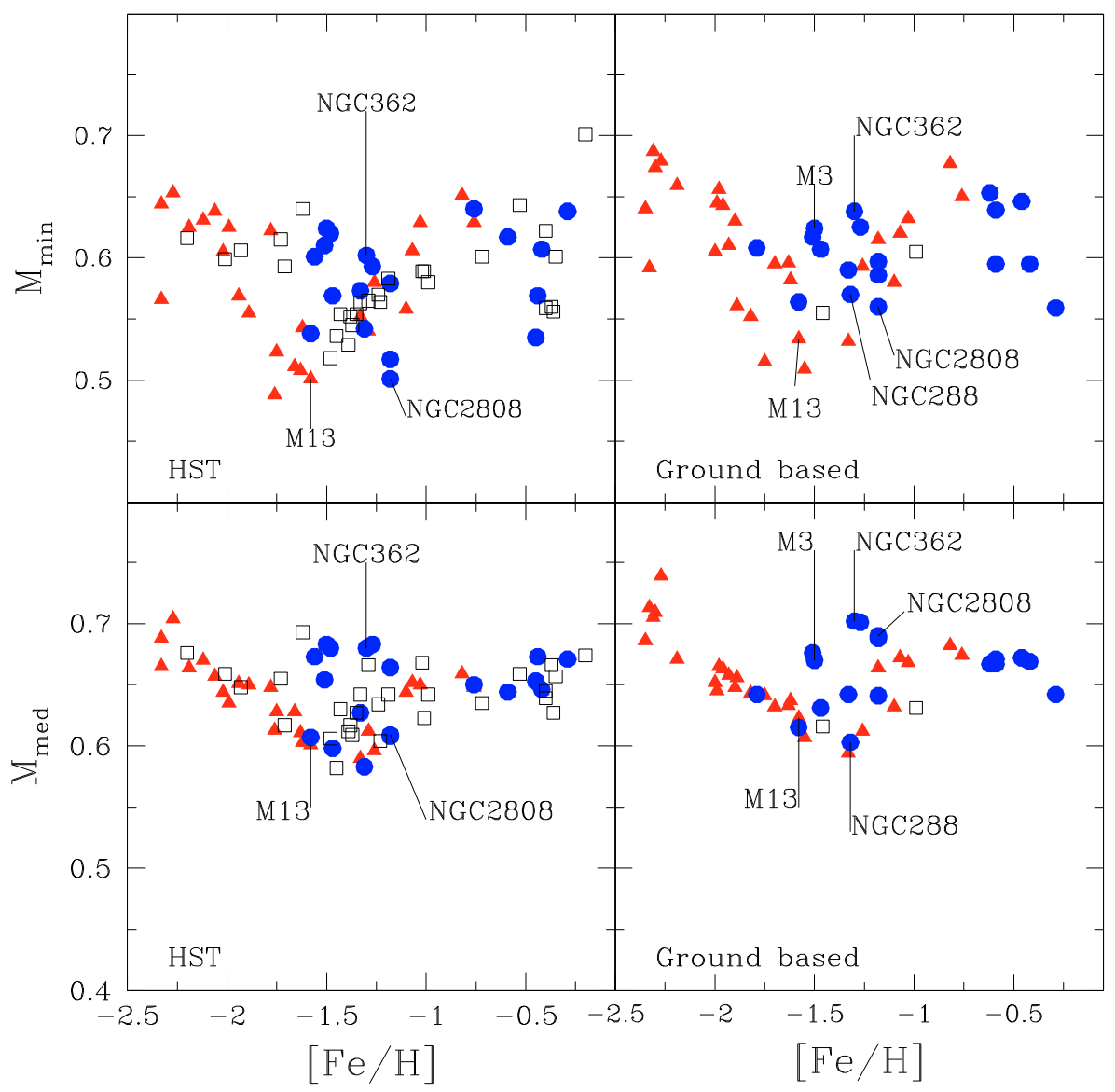

Fig. 10. Median and minimum masses vs. $[\mathrm{Fe} / \mathrm{H}]$ for HST and ground based samples. Symbol legend is given in Fig. 5. A few points relative to interesting clusters are marked. we used the relation

$$
\begin{aligned}
M_{\mathrm{TO}}= & 10^{-0.259 \log \mathrm{Age}}(0.9636+0.171[\mathrm{Fe} / \mathrm{H}] \\
& \left.+0.04073[\mathrm{Fe} / \mathrm{H}]^{2}\right) \quad M_{\odot}
\end{aligned}
$$

that closely follows the isochrones of the Pisa group when $Y=$ 0.25 , over the range of parameters (age, metallicity) appropriate for GC stars. We note that Age in this formula is the relative age parameter of Carretta et al. (2009d), and we assumed that Age $=1$ corresponds to an isochrone age of $12.5 \mathrm{Gyr}$. We then corrected this value to those appropriate for stars at the tip of the RGB (neglecting mass loss, hence assuming the mass of the stars to be identical to those at the beginning of their evolution), using the formula

$M_{\mathrm{RGB}}=M_{\mathrm{TO}}+0.056+0.0117[\mathrm{Fe} / \mathrm{H}] \quad M_{\odot}$,

which again fits the Pisa group isochrones.

We then assume that the original mass of stars at the tip of the RGB is equal to that of stars currently on the HB (on average, it should be slightly lower, but this difference can be neglected in our discussion). The median mass lost by the stars before reaching the HB can then be obtained by comparing their current mass $M_{\text {med }}$ (as determined in the previous paragraph) with $M_{\mathrm{RGB}}$. The precise evolutionary phase (before ZAHB) at which mass loss occurs is not important in our approach. It would of course play an important rôle in the comparison with mass loss laws. The values of the median mass loss for stars in each cluster are plotted against metallicity in Fig. 11 for clusters with HST (upper panel) and ground-based data (bottom panel). As usual, we used different symbols for old and young clusters (age is required when deriving this estimate of the mass loss, so that clusters with no age estimates cannot be plotted here).
Mass losses obtained from the two sets of data are quite similar, with a small offset of about $0.020 M_{\odot}$ between them. The best-fit straight line through the HST data is

$M_{\text {lost }}=(0.359 \pm 0.024)+(0.0942 \pm 0.0066)[\mathrm{Fe} / \mathrm{H}] M_{\odot}$,

which has a highly significant correlation coefficient of $r=0.91$ over 45 clusters. The scatter of points relative to each cluster around this mean line is small (rms of 0.021 and $0.027 M_{\odot}$ for old/young clusters, respectively) from HST data. The scatter is very similar $\left(0.022\right.$ and $0.025 M_{\odot}$ for old/young clusters, respectively) for ground-based data.

At first look, this small scatter appears to be compatible with errors in metallicity and ages that are used in this derivation. For instance, in the case of the metal-rich cluster NGC 6441, changing the assumed values of the metallicity $[\mathrm{Fe} / \mathrm{H}]$ and age parameter by 0.10 would cause variations in the values estimated for the mass loss by 0.014 and $0.031 M_{\odot}$ (larger mass losses are obtained if metallicity and ages are increased). Sensitivities to these parameters are smaller for more metal-poor clusters; e.g., in the case of NGC 7078, similar changes in the assumed values of metallicity and age would cause variations in the mass loss of 0.007 and $0.022 M_{\odot}$. In conclusion, a simple unique mass-loss law, combined with metallicity and age, seems able to define quite well the median mass and colours of HB stars for the whole sample of clusters considered in this analysis. However, as we will show in Sect. 4, the deviations of individual points from the mean relation are caused mostly by true physical effects.

We may compare this estimate for the total mass lost along the RGB with predictions by mass-loss laws in the literature. A compilation of these predictions is given in Table 1 and Fig. 4 of Catelan (2009a). The present estimate of the mass lost is generally much higher than the values given by these predictions; 


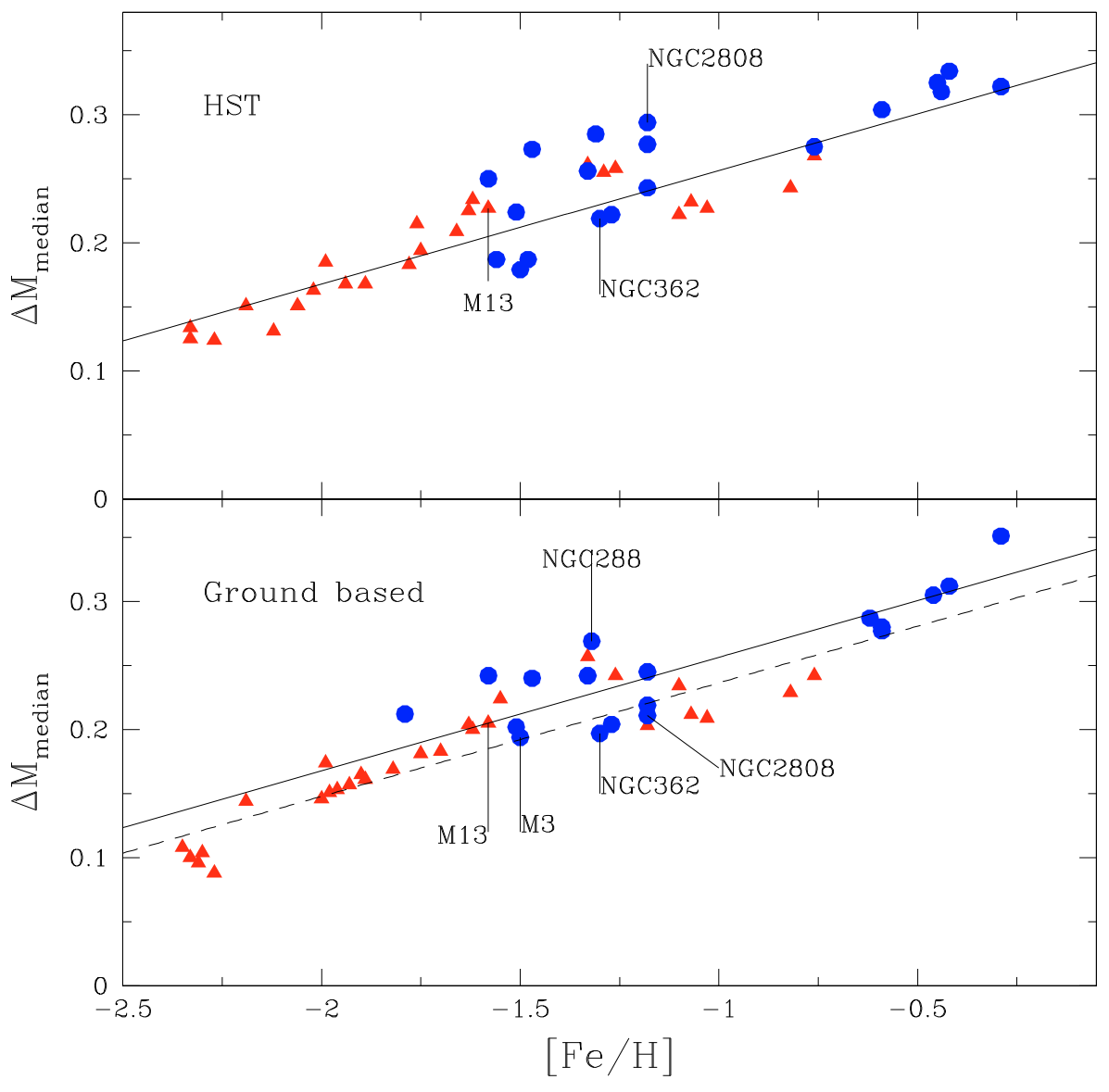

Fig. 11. Median mass loss as a function of metallicity for GCs older (triangles) and younger (dots) than 0.92 . The solid line is the best-fit relation to the HST data, while the dashed one is just shifted by $0.02 M_{\odot}$, reproducing rather well the ground-based data-set. A few points relative to interesting clusters are marked. only a small fraction $\left(0.002 M_{\odot}\right)$ of this difference can be attributed to the identification of the mass of $\mathrm{HB}$ stars with that of stars at the tip of the RGB. The observed run compares quite well with a simple or modified Reimers $(1975 \mathrm{a}, \mathrm{b}) \mathrm{laws}$, or the law by VandenBerg et al. (2000), provided that the efficiency parameter is roughly doubled. On the other hand, other mass-loss laws (Mullan 1978; Goldberg 1979; Judge \& Stencel 1991) provide a total mass lost along the RGB that changes too fast with metallicity to reproduce current results.

Origlia et al. (2007) proposed a mass-loss law based on mid-IR Spitzer observations of red giants in globular cluster. According to this mass-loss law, GCs star should lose about $0.23 M_{\odot}$ while ascending the RGB, nearly independent of their metallicity. While this average value roughly corresponds to what is needed to explain the HB of GCs of intermediate metallicity, our analysis indicates that the total mass lost along the RGB should depend on metallicity.

We note that the assumption of a particular age-metallicity relation (the one by Marin-Franch et al.) is implicit in our analysis. Had we adopted a different age-metallicity relation, the metallicity-mass-lost relation would be different. For instance, if the (relative) ages of disk-inner halo clusters were independent of metallicity (rather than with a slope of $\sim-0.1$ as adopted throughout this paper), the slope of the mass-loss law with metallicity would be reduced by $\sim 0.02 M_{\odot} / \mathrm{dex}$, from $\sim 0.09 M_{\odot} /$ dex to $\sim 0.07 M_{\odot} /$ dex. This would not modify significantly any of the main results of the paper.

\section{RR Lyrae}

RR Lyrae variables are HB stars within the classical instability strip. Properties of the pulsating stars may be used to constrain several basic properties, including mass, radius, and chemical composition. An in-depth star-by-star comparison, while very illuminating, requires a considerable effort, because individual HB stars may be within the instability strip at various phases of their HB evolution. This comparison then requires a synthesis of populations, such as those performed by Marconi et al. (2003), Di Criscienzo et al. (2004), Caloi \& D'Antona (2007, 2008), and D'Antona \& Caloi (2008). We defer this analysis to forthcoming papers; here we briefly examine only a few features.

\subsection{Data}

Because of the uncertainty related to evolution, we focus only on those clusters where the RR Lyrae population is dominated by stars still close to the ZAHB in the CMD. Since most of the lifetime of HB stars is spent close to their ZAHB location, we may be reasonably sure that this occurs for GCs that contain a large population of RR Lyrae. To identify these clusters, we consider the RR Lyrae richness parameter $S(\mathrm{RR})$ as tabulated in the Harris (1996) catalogue, which is the number of RR Lyrae per unit cluster luminosity 6 . In Fig. 12, we compared this parameter with the number of stars in the instability strip that we may obtain from the photometric catalogues considered in Sect. 2. We derived the number of RR Lyrae in each cluster by assuming that all $\mathrm{HB}$ stars whose dereddened $(B-V)_{0}$ colour is $0.15<(B-V)_{0}<0.45+0.024([\mathrm{Fe} / \mathrm{H}]+1.5)$ are RR Lyrae (see Marconi et al. 2003; and Di Criscienzo et al. 2004).

\footnotetext{
6 As pointed out by the referee, the value of $S$ (RR) listed in the Harris catalogue are underestimated for some GCs. A couple of examples are NGC 2808 (see Corwin et al. 2004) and M 62 (Contreras et al. 2005).
} 
Table 8. Properties of the RR Lyrae variables for each cluster.

\begin{tabular}{ccccccccc}
\hline \hline Cluster & $S(\mathrm{RR})$ & $n(\mathrm{RR})_{\mathrm{HST}}$ & $f(\mathrm{RR})_{\mathrm{HST}}$ & $n(\mathrm{RR})_{\mathrm{GB}}$ & $f(\mathrm{RR})_{\mathrm{GB}}$ & $f(\mathrm{RR})$ & $\left\langle P_{\mathrm{ab}}\right\rangle$ & $n\left(P_{\mathrm{ab}}\right)$ \\
\hline NGC 104 & 0.2 & 2 & 0.01 & 1 & 0.01 & 0.01 & 0.737 & 1 \\
NGC 288 & 4.0 & & & 3 & 0.04 & 0.03 & 0.678 & 1 \\
NGC 362 & 3.0 & 35 & 0.13 & 4 & 0.07 & 0.02 & 0.542 & 7 \\
IC 1257 & & 0 & 0.00 & & & 0.00 & & \\
NGC 1261 & 13.5 & 8 & 0.06 & 21 & 0.10 & 0.07 & 0.555 & 13 \\
\hline
\end{tabular}

Notes. The complete table is available at the CDS.

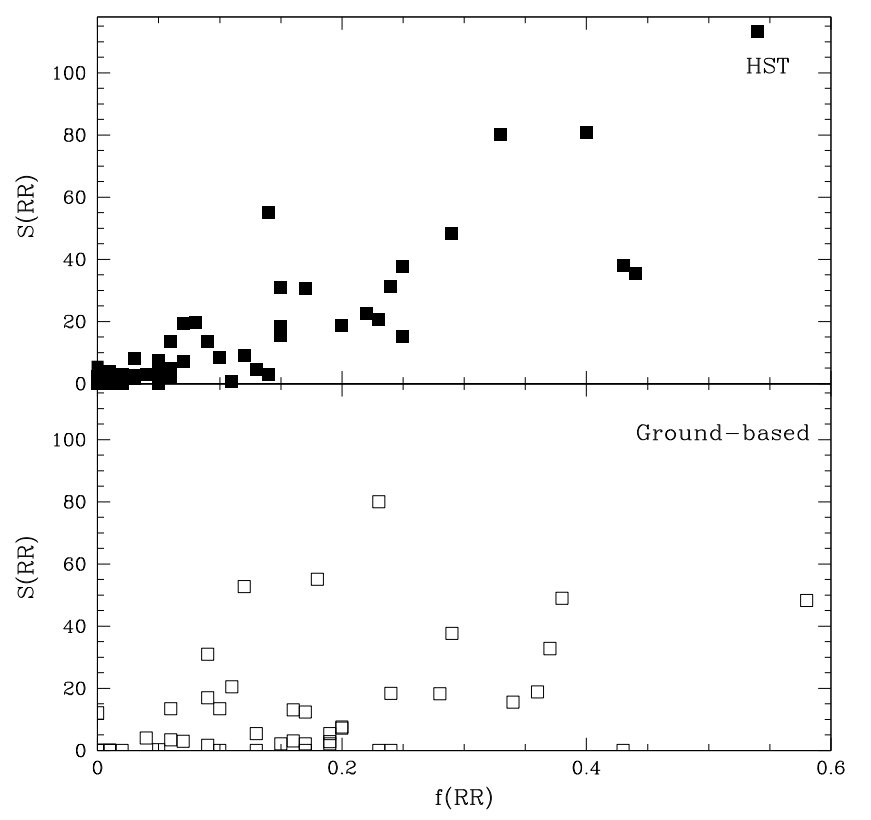

Fig. 12. RR Lyrae richness parameter, S(RR), as a function of the RR Lyrae fraction for both HST (upper panel) and ground-based sample (lower panel).

In Table 8, we report for each cluster the $S$ (RR) values (Col. 2) as well as the number and the fraction of RR Lyrae stars from both HST and ground-based samples (Cols. 3-6). In Col. 7, we list the $f(\mathrm{RR})$ estimates derived whenever possible from $S(\mathrm{RR})$ values, or otherwise from $f(\mathrm{RR})$, while the mean period $\left(P_{\mathrm{ab}}\right)$ and the corresponding number of stars $\left(n\left(\mathrm{RR}_{\mathrm{ab}}\right)\right)$ are reported in Cols. 8 and 9, respectively. These last two values are from Clement et al. (2001).

We note that the photometry considered throughout this paper is based on only a few, not even simultaneously acquired images in each filter. Hence, individual RR Lyrae will have nominal colours and magnitudes that can differ substantially from their mean values, and may even locate them out of our definitions of both the instability strip and the HB locus. However, we might expect that, provided that photometric errors are not too large, there should be a correlation between the number of stars that according to the photometric catalogues are within the instability strip, and the true number of RR Lyrae. This appears to be the case: the correlation between the fraction of HB stars that are nominally within the instability strip, and the RR Lyrae richness parameter listed by Harris, is quite good for HST data which have smaller photometric errors. It is somewhat poorer, but still fairly good for ground-based data. This correlation allows us to (i) calibrate the richness parameter in terms of the fraction of $\mathrm{HB}$ stars that are actually RR Lyrae $f(\mathrm{RR})$ (we found that this is represented by the mean relation
Table 9. Comparison of median and maximum masses with the masses for RR Lyrae variables derived by Di Criscienzo et al. (2004).

\begin{tabular}{cccr}
\hline \hline NGC & $\begin{array}{c}M_{\mathrm{RR}} \\
M_{\odot}\end{array}$ & $\begin{array}{c}M_{\text {med }} \\
M_{\odot}\end{array}$ & $\begin{array}{r}M_{\max } \\
M_{\odot}\end{array}$ \\
\hline 1851 & 0.66 & 0.666 & 0.688 \\
3201 & 0.69 & 0.654 & 0.706 \\
4499 & 0.70 & 0.693 & 0.733 \\
4590 & 0.80 & 0.704 & 0.756 \\
5272 & 0.69 & 0.651 & 0.698 \\
5466 & 0.74 & 0.685 & 0.738 \\
5904 & 0.66 & 0.627 & 0.687 \\
6362 & 0.66 & 0.652 & 0.686 \\
6809 & 0.69 & 0.638 & 0.676 \\
6934 & 0.70 & 0.673 & 0.705 \\
7078 & 0.77 & 0.688 & 0.767 \\
7089 & 0.66 & 0.628 & 0.699 \\
\hline
\end{tabular}

$f(\mathrm{RR})=0.01+0.00458 S(\mathrm{RR})$ ); and (ii) complement the RR Lyrae richness parameter with data obtained from the photometry of those few clusters where this datum was missing.

To estimate the separation between clusters whose RR Lyrae population is dominated by ZAHB objects rather than evolved ones, we considered in more detail a few interesting cases using synthetic HBs. We found that using parameters that provide an accurate distribution of stars along the HB, the fraction of RR Lyrae stars that were within the instability strip also when they were on the ZAHB is 0.67 for NGC $4590(f(\mathrm{RR})=0.23)$, 0.45 for NGC $7078(f(\mathrm{RR})=0.10)$, and close to zero for NGC $4833(f(\mathrm{RR})=0.04)$. We note however that the values of $f(\mathrm{RR})$ are quite uncertain, and that synthetic HBs need several parameters to reproduce various aspects of the colour distribution. The fractions cited above are then uncertain and should only be taken as indicative of a decreasing ratio of stars close to the ZAHB to the total of RR Lyrae variables with decreasing specific frequency; and that a value in the range of $f(\mathrm{RR}) \sim 0.04-0.1$ roughly corresponds to the transition between a population of RR Lyrae variables dominated by ZAHB stars to a population dominated by evolved objects.

Hereafter, we assume that all clusters for which $f(\mathrm{RR})>0.10$ (that is $S(\mathrm{RR})>19.7$ ) have an RR Lyrae population dominated by stars that are still close to their ZAHB location. While as mentioned this limit is rather arbitrary, we found very similar results with other values in the range $0.04-0.10$.

After identifying GCs for which we may assume that most RR Lyrae remain close to the ZAHB, we can perform a sanity check of the minimum, median, and maximum masses that we adopted for HB stars by comparing them with those derived from pulsational properties. Table 9 lists the different mass values for the GCs analysed by Di Criscienzo et al. (2004). We found that masses derived from pulsational properties are very 
R. G. Gratton et al.: The second and third parameters of the horizontal branch

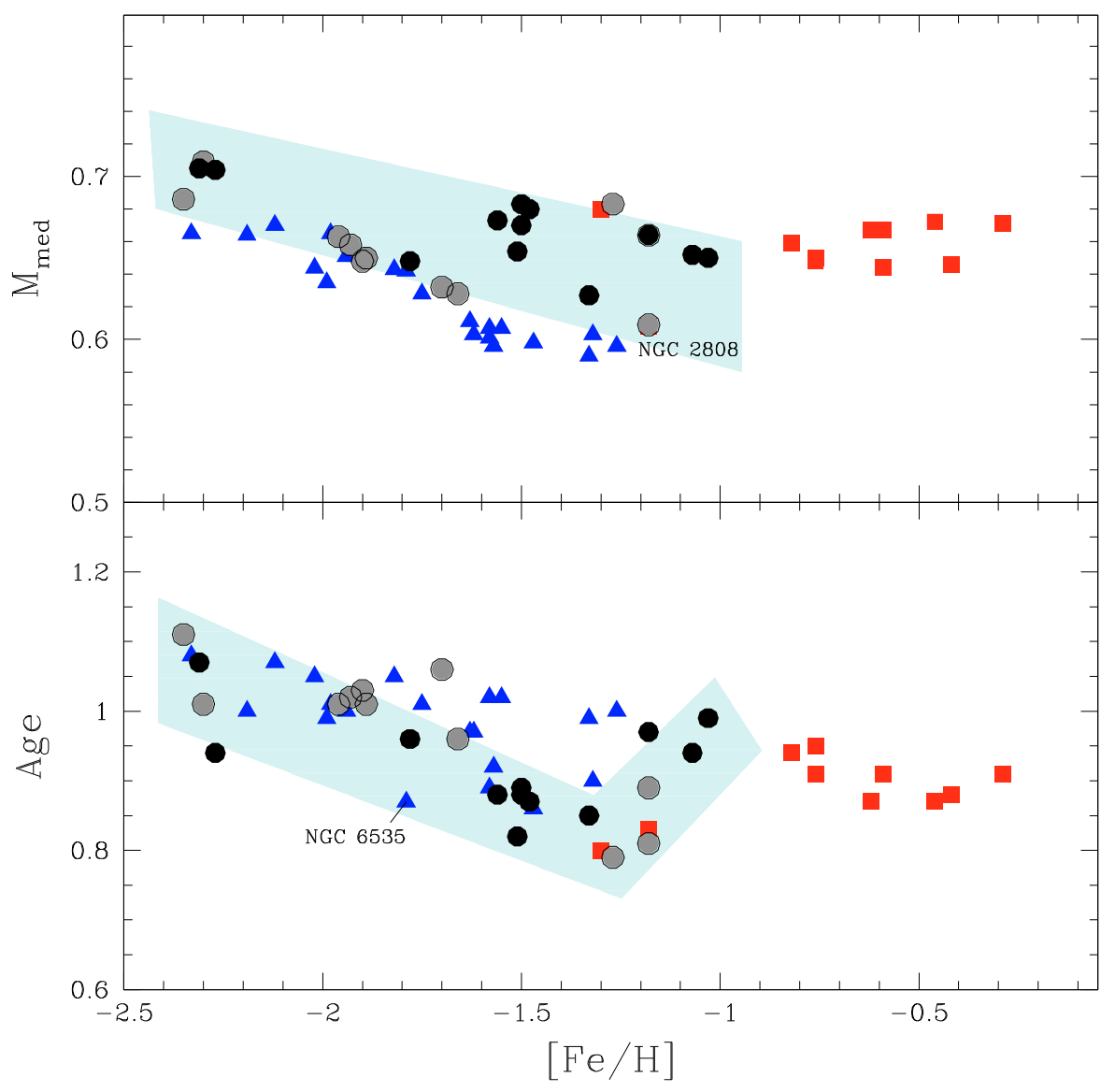

Fig. 13. Median mass and age as function of metallicity; different symbols in this case are for GCs with different RR Lyrae fractions (black: $f(\mathrm{RR})>0.10$, grey: $0.04<f(\mathrm{RR})<0.10)$ and HBR ratios (blue triangles: HBR $>0$, red squares: HBR $<0$ ). The shaded area is the strip containing GCs rich in RR Lyrae. similar to the median masses for the metal-rich clusters (which typically have an $\mathrm{HB}$ extending on both sides of the instability strip), and closer to the maximum masses for metal-poor clusters, which have predominantly blue HBs. While this result is unsurprising because we use the same HB evolutionary models as Di Criscienzo et al. (2004), who performed a similar comparison, at least it shows that there are no gross errors in the way we applied these models.

\subsection{Discussion}

One of the most intriguing characteristics of $\mathrm{HBs}$ is the dichotomy in the distribution of the GCs in terms of the mean periods of variables pulsating in the fundamental mode (RRab), discovered by Oosterhoff (1944). We note that while the Oosterhoff dichotomy applies not only to GCs, but also to field stars in our Galaxy (e.g., Szczygiel et al. 2009), populations of RR Lyrae sampled in other (smaller) galaxies such as the Magellanic Clouds or the dSphs have mean periods that are intermediate between the two Oosterhoff groups (OoI and OoII, e.g., Pritzl et al. 2004; Catelan 2009a). A quite extensive discussion of various properties of GCs in relation to the Oosterhoff dichotomy can be found in Sect. 6 of Catelan (2009a). The information collectively is quite difficult to interpret, because of significant uncertainties in many of the basic parameters considered (in particular, ages, but also metallicities), of small number statistics, and because average periods within a cluster depend on a complex combination of factors, mainly related to RR Lyrae possibly belonging to different evolutionary phases. In spite of this, many authors have searched for a simple explanation of the Oosterhoff dichotomy, and several of them suggested that this might be caused by a combination of ages and metallicities, the OoII group being attributable to old, metal-poor GCs, and the OoI to a population of young, metal-intermediate clusters (see e.g., Lee \& Carney 1999). We used our data to test whether this interpretation of the Oosterhoff dichotomy is consistent with the HB scenario we consider in this paper. We first replotted (upper panel of Fig. 13) the metallicity-median mass diagram of Fig. 10, but this time using different symbols for GCs that are either rich or poor in RR Lyrae: black and grey circles are for $f \geq 0.10$ and $0.04 \leq f<0.10$, respectively. The GCs with $f<0.04$ are divided according to the HBR ratios, (blue) triangles and (red) squares representing HBR greater and smaller than 0, respectively. Not unexpectedly, RR Lyrae-rich GCs occupy a well defined locus in this diagram, roughly represented by a diagonal strip; GCs below this strip have blue $\mathrm{HBs}$, while those above the strip have red HBs. We note that the anomalous behaviour of NGC 6535, being a BHB cluster with very low RR Lyrae fraction and located within the instability strip (shaded box) probably reflects a small amount of data in the CMD of this cluster.

As shown by Fig. 10, this diagram is essentially an agemetallicity diagram (with some caveats that are described more clearly in the next section). Given the distribution of GCs in age and metallicity, there are very few galactic GCs with young ages and $-2<[\mathrm{Fe} / \mathrm{H}]<-1.6$. This lack of GCs with suitable parameters divides the galactic population of RR Lyrae rich GCs into two ensembles, which can be identified with the Oosterhoff groups, confirming what had already been proposed by many other authors. However, ages of both groups have a much larger spread than given by this simple consideration. This is because of two results: (i) the diagonal strip defined by the RR Lyrae-rich clusters has a considerable width, much larger than that caused simply by the range in colour of the instability strip itself; this indicates that there is an intrinsic spread in the masses of ZAHB 
Table 10. Clusters' ages from main sequence fitting and from HB both for HST and ground based data-set. $Y_{\text {med }}$ is given in Table 12.

\begin{tabular}{lcccc}
\hline \hline Cluster & Age(MS) & $\begin{array}{c}\text { Age(HB) } \\
\text { HST }\end{array}$ & $\begin{array}{c}\text { Age(HB) } \\
\text { ground }\end{array}$ & $\operatorname{Age}(Y)$ \\
\hline NGC 104 & 0.95 & 0.877 & 0.856 & 0.922 \\
NGC 288 & 0.90 & & 1.175 & 0.958 \\
NGC 362 & 0.80 & 0.744 & 0.738 & 0.789 \\
IC 1257 & & 0.880 & & \\
NGC 1261 & 0.79 & 0.735 & 0.741 & 0.780 \\
\hline
\end{tabular}

Notes. The complete table is available at the CDS.

stars, which is a basic property of HBs that we exploit throughout this paper. This is important in particular for the case of metal-poor clusters, which are able to produce an RR Lyrae rich population over a rather wide range of ages. (ii) Given the interplay between the original mass and the mass-loss variation with metallicity, the strip occupied by RR Lyrae-rich clusters overlaps twice with the locus occupied by old GCs in the metallicitymedian mass diagram. This implies that there are old RR Lyraerich GCs that are either metal-poor (the classical Oo II clusters), or in a restricted range of metallicity around $[\mathrm{Fe} / \mathrm{H}]=-1$. Hence, the most metal-rich among the Oo I GCs belong to the old population, and are actually as old as the most metal-poor Oo II clusters. This is clearly shown in the age-metallicity diagram shown in the bottom panel of Fig. 13, where again we plotted with different symbols clusters that are either rich or poor in RR Lyrae.

This description only refers to those GCs that are very rich in RR Lyrae. There are many GCs with only a few RR Lyrae: as mentioned above, in these cases (generally metal-poor clusters with predominantly $\mathrm{BHB}$ ) most variables can be identified with stars that have evolved off the ZAHB. In these cases, we should expect long mean periods, and the cluster to be identified as Oo II (e.g., Lee et al. 1990). Furthermore, there are peculiar cases (including e.g., NGC 6388 and NGC 6441) where the RR Lyrae are actually HB stars with masses much lower than expected for their metallicity and age (see next section). All these cases complicate the simple picture described above, as discussed by Catelan (2009a).

To summarise, although the Oosterhoff dichotomy indeed depends on the peculiar distribution of galactic GCs in the agemetallicity plane, and most of the Oo II clusters are old while most of the Oo I clusters are young, it is not possible to identify tout-court the Oosterhoff groups with groups of GCs having different ages (a similar conclusion was drawn by Catelan 2009a).

\section{Ages from median HB colours}

After demonstrating that the median colour of the HB is determined mainly by cluster metallicity and age, provided a suitable mass-loss law is adopted, we attempt to derive ages for GCs from their median HB colour. This procedure is almost identical to that considered by several authors (e.g., Lee et al. 1994), but it is now applied to a large database of GCs in a uniform way.

These ages can be obtained by simply adding to the median mass of the HB stars the mass lost during the previous evolution (Eq. (11)), then subtracting the mass difference between stars at the tip of the RGB and the TO (Eq. (10)), and finally inverting the age-metallicity-TO mass relation (Eq. (9)). Of course, since the mean mass-loss law adopted throughout this paper (a linear dependence on $[\mathrm{Fe} / \mathrm{H}]$ ) was obtained by comparing median masses on the HB with those at the tip of the RGB, where mass loss is neglected, on average the two sets of ages

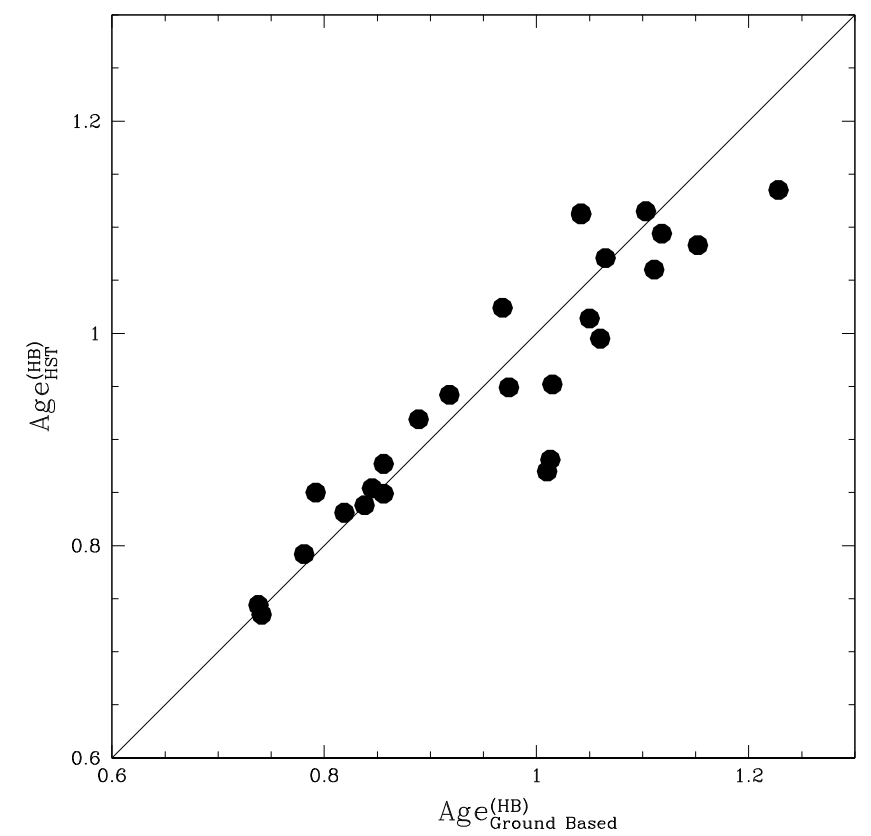

Fig. 14. Comparison of ages derived from HB for HST and groundbased samples.

should agree. However, any difference in the ages derived from the HB and RGB for a given GC indicates peculiarities for that GC. Taking an extreme view, were not age the main parameter affecting the HB morphology, we might have no correlation (or even an anticorrelation) between MS and HB ages, only their mean values being in agreement. The derivation of ages from $\mathrm{HB}$ is then crucial to understanding the second parameter issue. These ages (expressed in our usual relative scale) are listed in Cols. 3 and 4 of Table 10 for HST and ground-based data, respectively. We compared these two age estimates in Fig. 14. The two sets of ages agree very well each other, with the exception of NGC 2808, for which the ground-based HB age is underestimated because the limiting magnitude of the relevant photometry is too bright to include the faint end of the HB. Once this cluster is dropped, the mean difference is $0.003 \pm 0.009$ $(\mathrm{rms}=0.046)$; assuming the same error in both age estimates, this implies an internal error of 0.032 for each of them. In the following, we adopt as our most robust estimates of the HB ages those determined from HST data, whenever available, otherwise we use values obtained from ground-based data.

In Fig. 15, we compare these HB ages with those determined from the main sequence. The agreement is fairly good: the rms of the difference in ages from HB and from the main sequence is 0.094 for HST and 0.105 for ground-based data respectively: this is approximately equivalent to $1 \mathrm{Gyr}$. While this agreement may appear satisfactory, it is actually much larger than internal error bars ${ }^{7}$.

There are various ways to convince ourselves that this is indeed the case. First, we compared in Fig. 16 the HBs of two GCs (NGC 1904 and NGC 6934) that have very similar metallicities $([\mathrm{Fe} / \mathrm{H}]=-1.58$ and $[\mathrm{Fe} / \mathrm{H}]=-1.56$, respectively) and relative ages from MS (0.89 and 0.88). It is clear that their HBs are vastly different. The median mass values we obtain are $0.606 \pm 0.003$ and $0.671 \pm 0.008$ for NGC 1904 and NGC 6934,

7 This indicates that the scatter about the mean mass-loss relation in Fig. 11 is indeed real, as mentioned in Sect. 2.4, and not simply due to observational errors. 
R. G. Gratton et al.: The second and third parameters of the horizontal branch

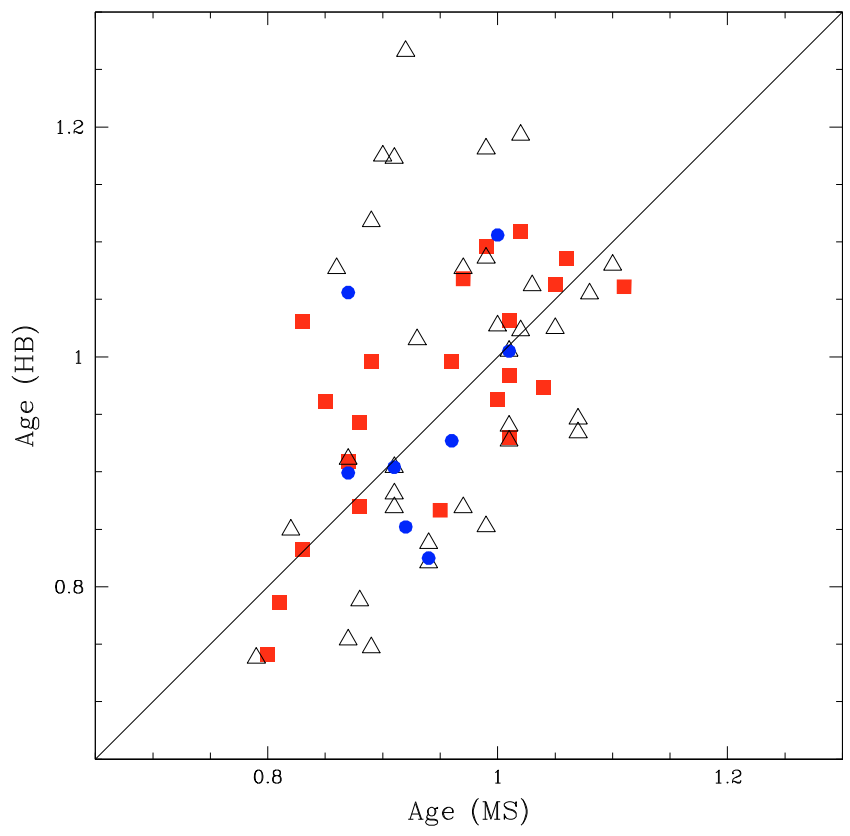

Fig. 15. Comparison of ages from HB and from main sequence for both HST and ground based samples; filled squares and circles are GCs with $M_{\mathrm{V}}<-8 \mathrm{mag}$ and $M_{\mathrm{V}} \geq-6.5$, respectively, while empty triangles are for $-8.0 \leq M_{\mathrm{V}}<-6.5$. Note that the scatter around a 1:1 correlation is larger for clusters of intermediate luminosity (see text).

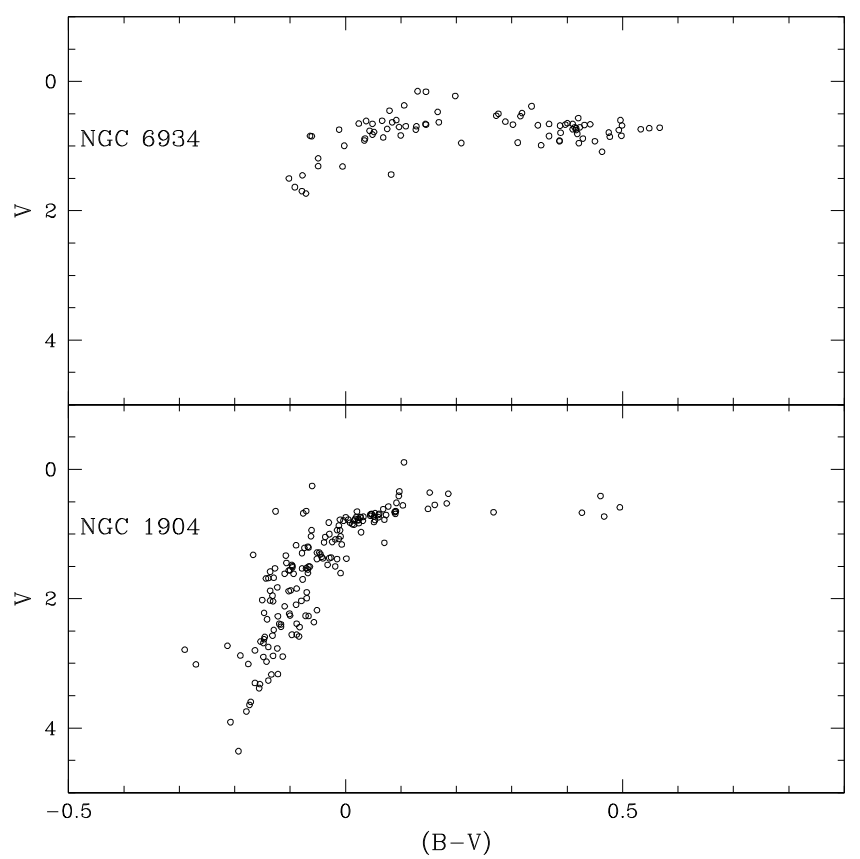

Fig. 16. Dereddened HST CMDs for stars on the HB of NGC 6934 and NGC 1904.

respectively. The two clusters also have a very different frequency of RR Lyrae, i.e., $f(\mathrm{RR})=0.02$ and 0.38 .

Second, as shown by Fig. 15, the scatter of ages from MS and from HB is much larger for GCs of intermediate luminosity and smaller for both brighter and fainter clusters. Furthermore, there is a clear trend of this scatter with metallicity. This is shown in Fig. 17, where GCs of different metallicity are plotted separately in the three panels. In this figure, we also plotted the typical error bars as well as arrows showing the impact of errors in the assumed metallicity and He content. These errors take consistently

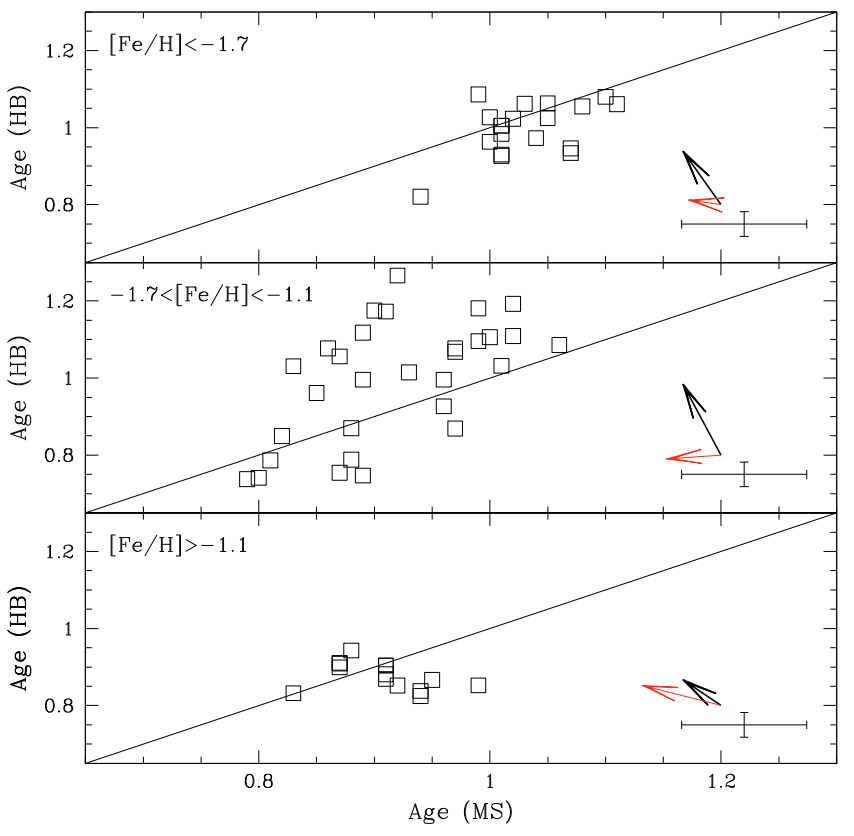

Fig. 17. Comparison of ages from $\mathrm{HB}$ and from main sequence for the whole sample (HST+ground based); typical error bars are shown at bottom right corner of each panel. The thick (black) arrows show the impact of a 0.02 variation in the assumed He content, while the thin (red) ones represent the consequence of a 0.1 dex error in $[\mathrm{Fe} / \mathrm{H}]$.

into consideration the impact of the He content on the MS colour, the TO point, and the HB. While the uncertainties in the assumed metallicity have a relatively moderate impact on these age estimates, those in the He content very significantly affect the age estimates from HB. This effect is particularly large at intermediate metallicity, which is where we found the largest discrepancies between the two sets of ages. This suggests that the He content might be responsible for these discrepancies (although we cannot exclude that other terms, e.g., the total CNO abundances, might contribute). We also note that the errors in the ages from MS and HB due to He are anticorrelated with each other: if the true He abundances are higher than we assumed, the MS ages are underestimated, while the HB ages are overestimated. This result could be exploited to simultaneously derive the values of $Y_{\text {med }}$ and age for each cluster, by ensuring agreement between these two age derivations. The age values, $\operatorname{Age}(Y)$, obtained by applying this procedure are listed in the last column of Table 10. Age $(Y)$ values are typically close to MS ages, the largest differences being for a few clusters with very blue HBs, such as NGC 6254 and NGC 1904.

In principle, errors in $\mathrm{Age}(Y)$ should be obtained by quadratically summing the internal errors in MS and HB ages; the mean quadratic value determined in this way is 0.063 . To confirm the reliability of this uncertainty estimate, we plot the agemetallicity relation for different groups of GCs in Fig. 18: outer halo GCs, inner halo GCs, disk/bulge GCs, and GCs associated with dSphs (for the classification of the GCs in these different populations see Carretta et al. 2009d). Briefly, we replot the same age-metallicity diagram, as previously done in Carretta et al. (2009d) but with the new age values, i.e. $\operatorname{Age}(Y)$ : we found that for the disk/bulge GCs the relation becomes tighter, with an $\mathrm{rms}$ around a least squares fit of 0.036 with respect to 0.042 previously derived (note that assuming the age values directly retrieved from Marín-Franch et al. 2009, the scatter around the fit line is $\sim 0.050$ ). This improvement also supports the identification of this third parameter with variations in the median 


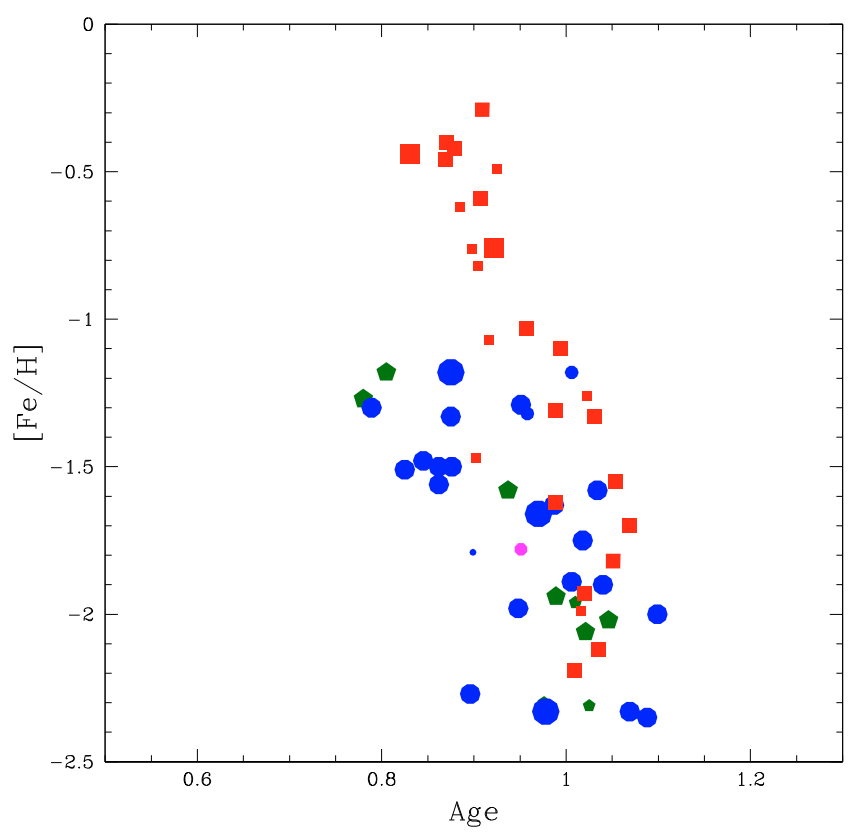

Fig. 18. Age-metallicity relation for different groups of globular clusters: outer halo clusters (green pentagons), inner halo clusters (blue circles), disk/bulge clusters (red squares). Magenta circles are GCs associated with dSphs. Different symbol sizes are used for clusters of different luminosity.

He abundances. Furthermore, the very small dispersion, well within the above cited error of 0.063 , suggests that the observational uncertainties in the ages from MS's were most likely overestimated. We consider these $\operatorname{Age}(Y)$ values to be the most robust estimates of the relative ages currently available: they incorporate updated photometric data and metallicities, and take into account cluster-to-cluster variations in the He content. We however postpone to a future paper a full discussion of the agemetallicity relation for the different Galactic populations of GCs.

\section{He from HB and $\boldsymbol{R}$-parameter}

Are there other arguments supporting the idea that the third parameter required to explain the median colours and masses of $\mathrm{HB}$ stars is indeed the He content? Is there any available evidence on variations in the median He content between different GCs? The most classical derivation of He abundances for GCs is obtained using the so-called $R$-parameter method (Iben 1968). The $R$-parameter is the ratio of the number of HB stars, $N_{\mathrm{HB}}$, to the number of RGB stars brighter than the HB in $M_{\mathrm{Bol}}, N_{\mathrm{RGB}}$. This ratio depends on the He content, because the higher the He content, the brighter the $\mathrm{HB}$, the smaller the number of RGB stars brighter than this level, and hence the larger the value of $R$. The $R$-parameter can be calibrated using models that include the variation in the lifetime of RGB and HB stars with metallicity. The most updated calibration of $R$ is that by Cassisi et al. (2003). This calibration was used by Salaris et al. (2004) to produce $R$ values and average He abundances for 57 GCs based on the same HST photometric database considered here. Salaris et al. (2004) set an upper limit to the rms scatter of $Y$ among these GCs of 0.019. By itself, this upper limit to the rms scatter is fully consistent with the rms scatter of 0.014 for $Y_{\text {med }}$ that we derived by matching $\mathrm{HB}$ and MS ages. It would be rewarding to identify some degree of correlation between the $Y$ values of individual GCs derived by the two techniques. However, the correlation is actually poor, probably because the $Y$ values, derived using the $R$-method, have large error bars. This is mainly due
Table 11. $R^{\prime}$ parameter as derived from both HST and ground based data (note the different definition with respect to the usual $R$ parameter).

\begin{tabular}{lcccc}
\hline \hline NGC /IC & $R^{\prime}$ (HST) & $R^{\prime}$ (ground based) & $\left\langle R^{\prime}\right\rangle$ & $Y\left(\left\langle R^{\prime}\right\rangle\right)$ \\
\hline 104 & $0.69 \pm 0.07$ & $0.68 \pm 0.09$ & $0.69 \pm 0.07$ & $0.253 \pm 0.012$ \\
288 & & $0.69 \pm 0.14$ & $0.69 \pm 0.14$ & $0.250 \pm 0.024$ \\
362 & $0.75 \pm 0.08$ & $0.63 \pm 0.15$ & $0.75 \pm 0.08$ & $0.260 \pm 0.012$ \\
1257 & $0.49 \pm 0.14$ & & $0.49 \pm 0.14$ & $0.213 \pm 0.024$ \\
1261 & $0.62 \pm 0.10$ & $0.69 \pm 0.09$ & $0.62 \pm 0.10$ & $0.238 \pm 0.017$ \\
\hline
\end{tabular}

Notes. The final adopted value $\left\langle R^{\prime}\right\rangle$ is the weighted average between the two estimates, when available. $Y\left(\left\langle R^{\prime}\right\rangle\right)$ is the corresponding He abundance. The complete table is available at the CDS.

to limited statistics, though in a few cases other error sources (contamination by field stars, differential reddening, photometric limits) contribute to the noise in $Y$ determinations.

We note that the statistical error in the $R$ parameter can in principle be reduced by adopting a different definition. With the usual definition, on average $R \sim 1.5$, there are more stars on the HB than on the RGB brighter than the HB, which is then a major contributor to the random noise. However, we may use a different definition of $R$, where the error caused by the small number of stars on the RGB is substantially reduced, but has a similar dependence on the helium content than the $R$-parameter. In practice, we may define $R^{\prime}=N_{\mathrm{HB}} / N_{\mathrm{RGB}}^{\prime}$, where $N_{\mathrm{RGB}}^{\prime}$ is the number of stars on the RGB brighter than $V(\mathrm{HB})+1$. Roughly, $N_{\mathrm{RGB}}^{\prime}$ is twice the value of $N_{\mathrm{RGB}}$, so that $R^{\prime}=N_{\mathrm{HB}} / N_{\mathrm{RGB}}^{\prime} \sim 0.7$. It is then clear that the statistical errors in $R^{\prime}$ are smaller than in $R$. Furthermore, checks of the stellar models of Bertelli et al. (2008) indicate that $R^{\prime}$ has roughly the same (fractional) dependence on $Y$ as $R$, with only one important modification: the RGB bump is brighter than $V(\mathrm{HB})+1$ in all GCs considered in this paper. Hence, $R^{\prime}$ has a simpler (roughly linear) dependence on $[\mathrm{Fe} / \mathrm{H}]$, unlike $R$. We then decided to repeat the test using $Y$ values inferred from $R^{\prime}$, rather than $R$.

Table 11 gives the values of $R^{\prime}$ deduced from HST and ground-based data; since completeness is more of a concern for this second set of photometry, we give preference to the HST values, whenever possible. When making this derivation, we simply used the HB level listed by Harris (1996). The errors related to individual entries of Table 11 are simply those from statistics, and do not include the effects of uncertainties in decontamination by field stars, differential reddening, and photometric limits. In a few cases, these effects are known and can be taken into consideration. On this basis, we exclude from our estimates the ground-based observations for NGC 2808, NGC 5986, and NGC 6266, and the HST data for NGC 2419, where the photometry is not deep enough to reach the extreme hot/faint tail of the HB. For NGC 5927, the ground-based photometry is also not good enough to discriminate between HB and RGB stars. The $R^{\prime}$ values are underestimated in these cases, and we did not consider them in our discussion.

In Fig. 19, we compare the average values of $R^{\prime}$ with the values of $R$ obtained by Salaris et al. (2004). There is a good correlation between these two sets of data, which improves if we consider the offset between metal-rich and metal-poor clusters caused by the luminosity of the RGB bump; however, we recall that this new determination is not completely independent of the Salaris et al. one, since both use the HST snapshot photometry. A reasonable good correlation is also obtained with the older determinations of $R$ by Sandquist et al. (2000) and Zoccali et al. (2000). 
R. G. Gratton et al.: The second and third parameters of the horizontal branch

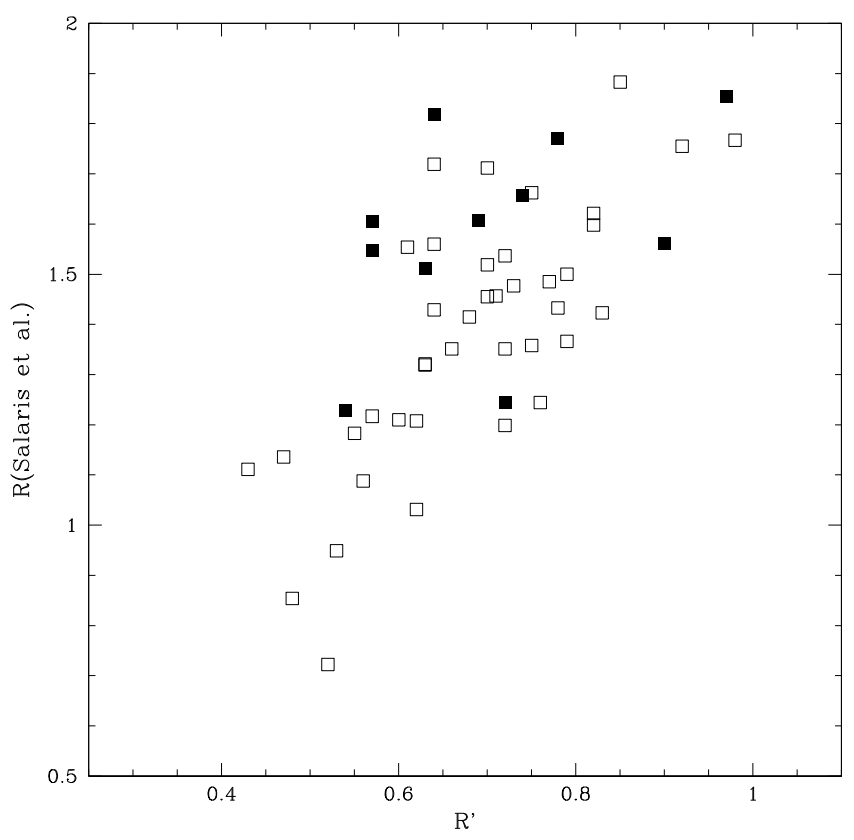

Fig. 19. The $R^{\prime}$ values plotted against the $R$ values from Salaris et al. (2004); filled and empty squares are for GCs with $[\mathrm{Fe} / \mathrm{H}]$ larger and smaller than -1.0 dex, respectively. Note the different definitions of $R^{\prime}$ and $R$ (see text).

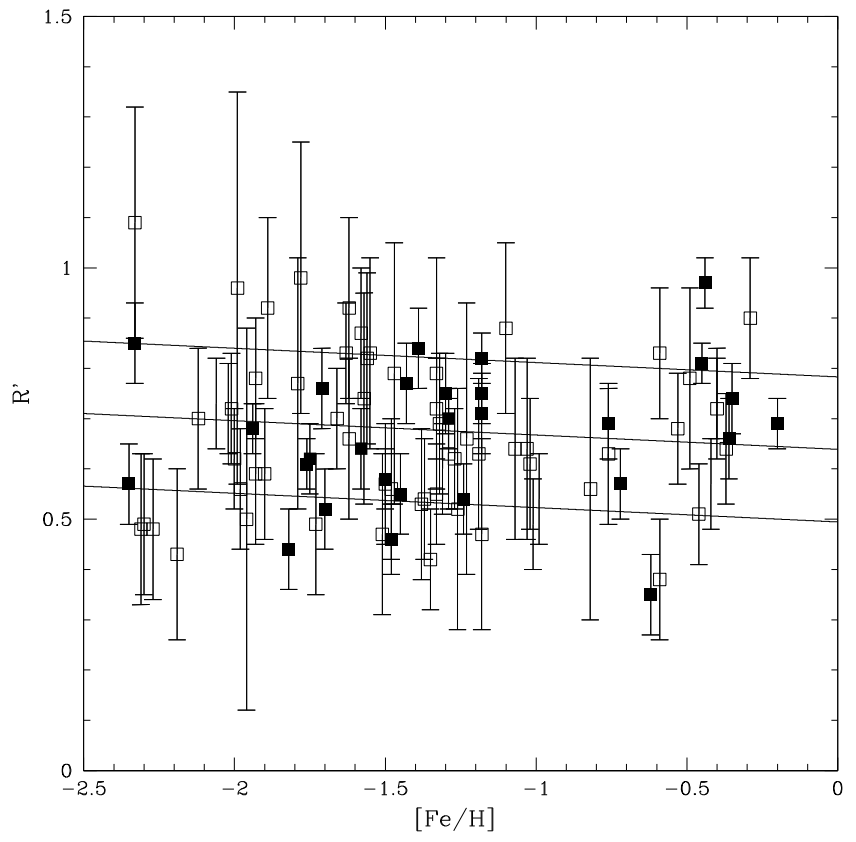

Fig. 20. The $R^{\prime}$ values plotted against $[\mathrm{Fe} / \mathrm{H}]$; filled and open symbols are GCs with or without age estimate, respectively. The solid lines correspond to $Y=0.225,0.250,0.275$.

The last column of Table 11 gives the value of $Y$ that we could derive from these $R^{\prime}$. These were obtained by scaling the calibration of Cassisi et al. (2003) for the ratio of $N_{\mathrm{RGB}}^{\prime}$ to $N_{\mathrm{RGB}}$, and taking into account that the RGB bump is brighter than $V(\mathrm{HB})+1$ in all GCs, while being fainter than $V(\mathrm{HB})$ for GCs with $[\mathrm{Fe} / \mathrm{H}]>-1.1$. In Fig. 20 we have plotted the values of $R^{\prime}$ against the metallicity $[\mathrm{Fe} / \mathrm{H}]$, and compared them with the calibrations we used. We note the absence of an obvious bump at $[\mathrm{Fe} / \mathrm{H}] \sim-1.1$, which is present in the case of the $R$-parameter.

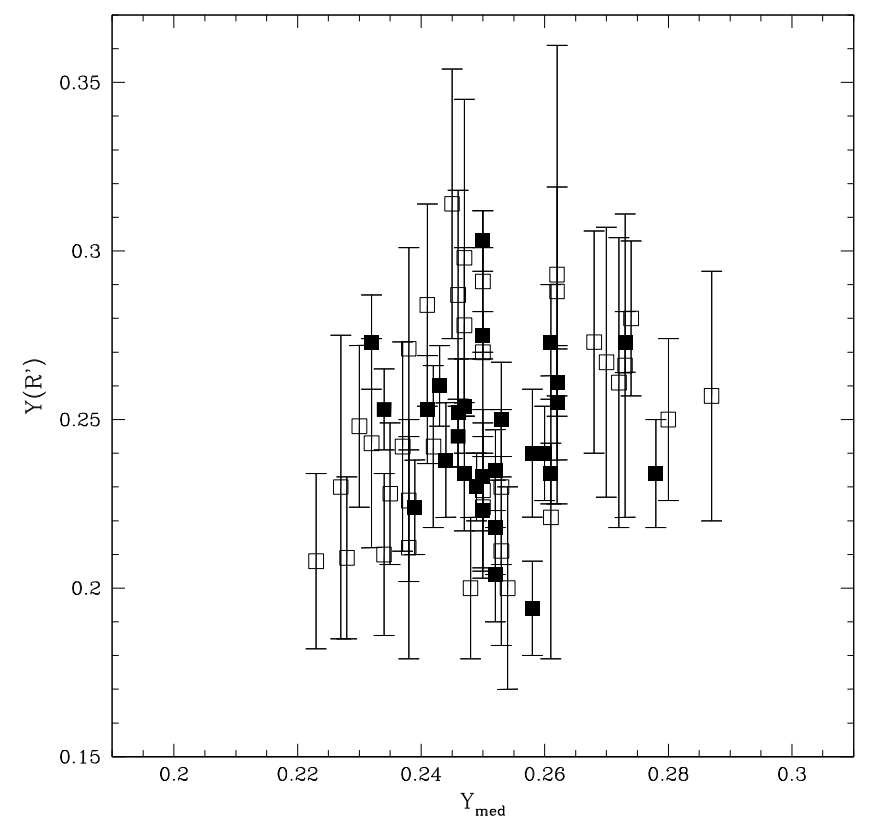

Fig. 21. The values of $Y_{\text {med }}$ are plotted against $Y\left(R^{\prime}\right)$; filled and open squares are for errors smaller and larger than 0.02 , respectively.

In Fig. 21, we plotted the values of $Y\left(R^{\prime}\right)$ obtained following this procedure against the median He abundance $Y_{\text {med }}$ required by placing ages estimated from the HB in agreement with those determined from the MS. There is some correlation between the two determinations of $Y$ (linear correlation coefficient $r=0.23$ over $64 \mathrm{GCs}$, which is significant at a level of confidence of $>95 \%)$. The scatter is clearly not negligible, as expected based on the rather large errors in $Y\left(R^{\prime}\right)$. However, we note that the meaning of these two quantities is not necessarily the same. To show this, we note that the strongest contribution to a $\chi^{2}$ test performed by comparing the two sets of He determinations is from NGC 6388 and NGC 6441; in both these clusters, $Y\left(R^{\prime}\right)$ is far larger than $Y_{\text {med }}$ than the (statistical) errors. The large value of $Y\left(R^{\prime}\right)$ is explained by $\mathrm{HB}$ at the RR Lyrae colour being very bright in these clusters (and indeed the RR Lyrae have anomalously long periods: Pritzl et al. 2002, 2003). On the other hand, the RR Lyrae are most likely in the tail of the distribution of high He abundances in these clusters, whose HB is predominantly red. In these cases, $Y\left(R^{\prime}\right)$ is then expected to be larger than $Y_{\text {med }}$, as is indeed observed. We also note that a much stronger correlation exists between $Y\left(R^{\prime}\right)$ and the average of $Y_{\text {med }}$ and $Y_{\max }$. In this case, the correlation coefficient is $r=0.294$ for the same 64 GCs, which is significant at a level of confidence of $\sim 99 \%$.

We conclude that the hypothesis that the He abundances obtained by matching ages from MS and HB is compatible with current estimates of the He abundances from the $R$-method, but that more extensive data sets and more careful comparisons (taking into consideration other possible indication of the He abundance, see e.g., Catelan et al. 2009-c) are needed to definitively settle this issue.

\section{The colour and mass spread along the HB 6.1. Observational data}

The previous discussion suggests that at least three parameters (metallicity, age, and most likely helium content) are required to provide a detailed explanation of the median colours of the HBs. We intend to demonstrate that this selection of parameters successfully explains the extension of the HBs. 


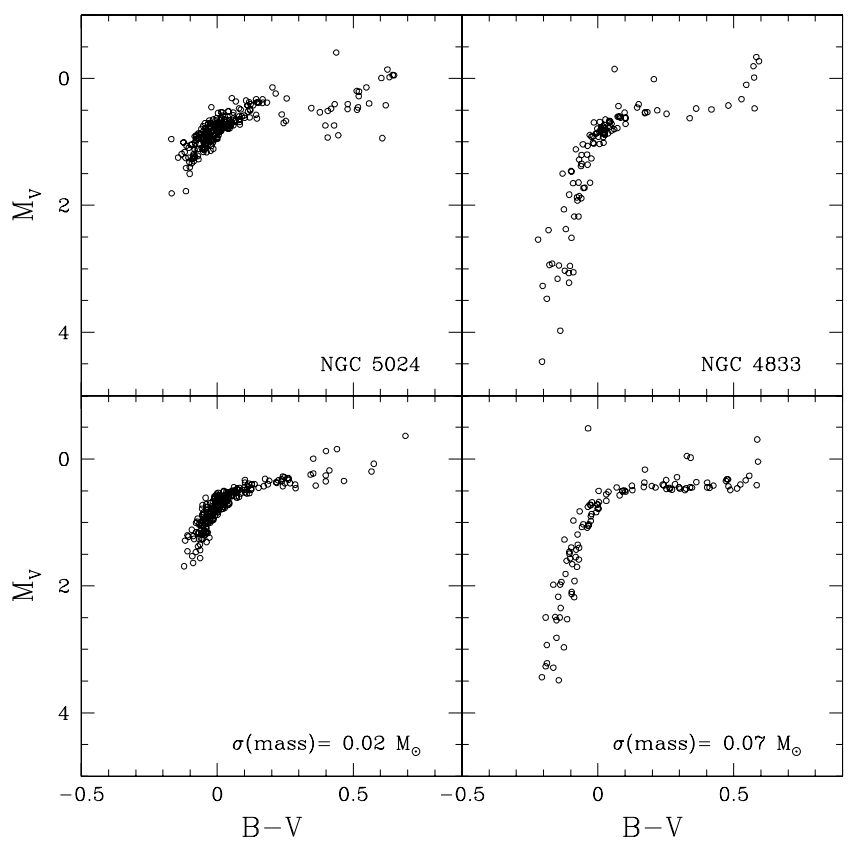

Fig. 22. Observed CMDs for NGC 4833 and NGC 5024 (upper panels) and synthetic diagrams lower panels), obtained with a Gaussian-like distribution mass loss with standard deviation of $0.02 M_{\odot}$ and $0.07 M_{\odot}$, respectively.

To illustrate our approach, in Fig. 22 we compare the $B V$ colour magnitude diagrams of NGC 4833 and NGC 5024. These two clusters have very similar ages (1.01 and 1.04, respectively) and metallicity $([\mathrm{Fe} / \mathrm{H}]=-1.89$ and -2.06 respectively), similar values of both median colour and mass of stars on the HB $\left(0.655\right.$ versus $\left.0.658 M_{\odot}\right)$, but yet very different HBs. The main difference between these two clusters is in the minimum mass of stars along the HB ( 0.561 vs. $\left.0.649 M_{\odot}\right)$. We can explain the short HB of NGC 5024 in terms of a small star-to-star scatter in mass loss (see e.g., Rood et al. 1973, and many others since). The bottom left panel of Fig. 22 shows a synthetic HB obtained by assuming that the mass lost during the RGB phase is distributed like a Gaussian, with a standard deviation of $0.02 M_{\odot}$ (this is the minimum value required to reproduce the shortest HBs). While this synthetic HB matches well the one observed in NGC 5024, it is clearly a poor match to that of NGC 4833, because it fails to describe its very extended faint tail. The difference between the two clusters is not simply in terms of the spread of mass loss. This is shown in the bottom right panel of Fig. 22, where we plotted a synthetic HB obtained assuming a mass loss spread of $0.07 M_{\odot}$, required for an HB extending as faint as the observed one for NGC 4833. However, this synthetic HB has by far too many red stars, as shown by a comparison of the cumulative distributions in colours (see Fig. 23) ${ }^{8}$. A Kolmogorov-Smirnov test shows that the observed colour distribution disagrees with that of the synthetic HB (at about $98 \%$ level of confidence). In contrast, a much better agreement is obtained between the observed and synthetic HBs for NGC 5024. We may conclude that at least

\footnotetext{
8 Small excesses of red stars along the HB - much smaller than found for NGC 4833 - are found often in our comparisons with synthetic HBs. This same problem was found by other similar analysis (see the discussion in Castellani et al. 2005). It might indicate either a deficiency of the adopted HB models, or a non-Gaussian distribution of the random terms of the mass loss, perhaps because it would be more appropriate to consider a Gaussian distribution in the mass-loss efficiency parameter (D’Cruz et al. 1996).
}
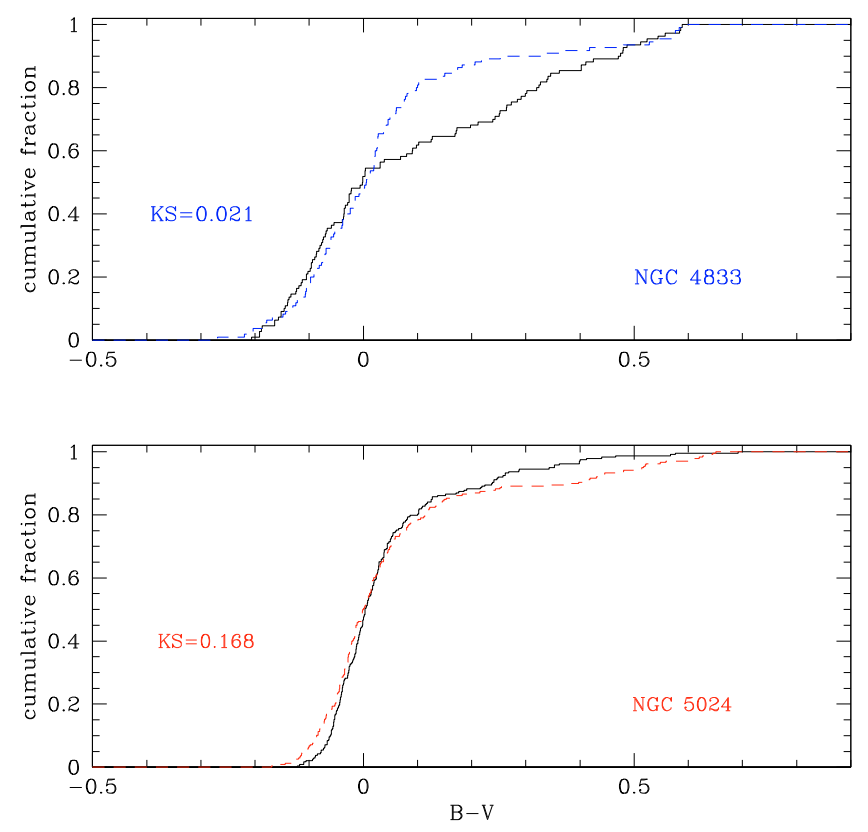

Fig. 23. Cumulative distribution of $(B-V)$ colours from colour-magnitude (dashed) and synthetic (solid) diagrams for NGC 5024 and NGC 4833; the resulting probabilities from Kolmogorov-Smirnov (KS) test are also shown.

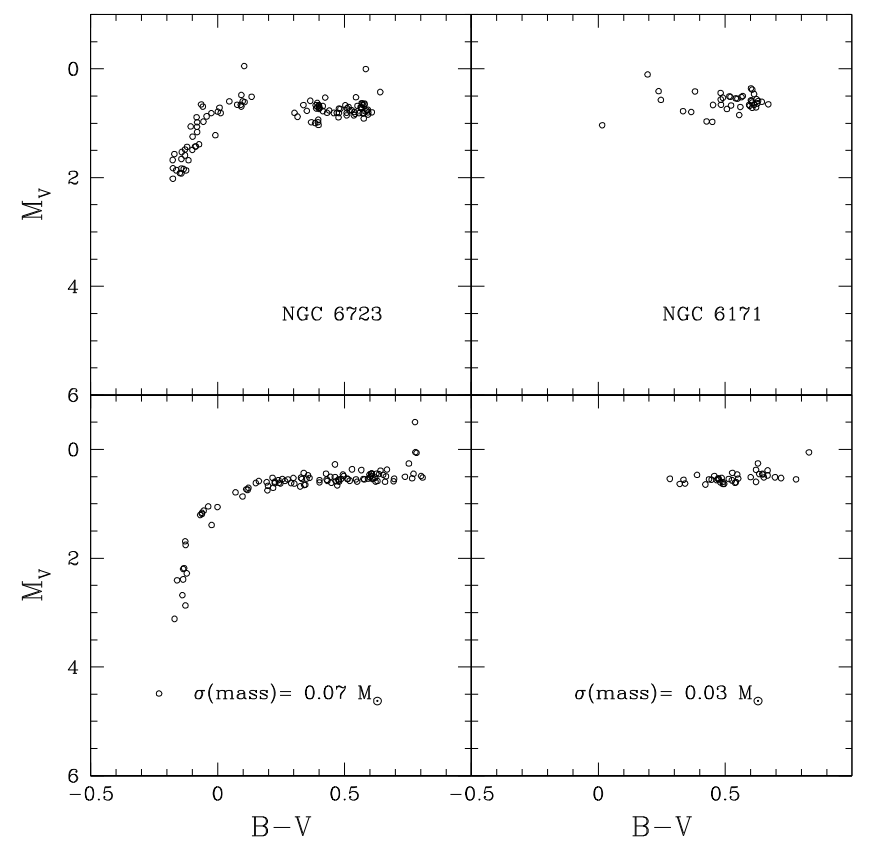

Fig. 24. Same as Fig. 22, but for the GCs NGC 6171 and NGC 6723. The synthetic diagrams (lower panels) were obtained with a Gaussianlike distribution mass loss with standard deviation of $0.03 M_{\odot}$ and $0.07 M_{\odot}$, respectively.

in the case of the pair NGC 4833-NGC 5024, a third parameter other than age and metallicity is required, and that this parameter mainly affects the minimum mass along the HB.

A similar comparison can be made using more metal-rich clusters. For this purpose, we selected the pair NGC 6171NGC 6723, which also have very similar metallicities and ages $([\mathrm{Fe} / \mathrm{H}]=-1.03$ and -1.10, Age $=0.99$ and 1.01, respectively $)$, similar median mass on the $\mathrm{HB}\left(0.650\right.$ and $\left.0.644 M_{\odot}\right)$, but again vastly different $\mathrm{HB}$, due to a very different value for the minimum mass $\left(0.629\right.$ vs. $\left.0.558 M_{\odot}\right)$. We can reproduce the 


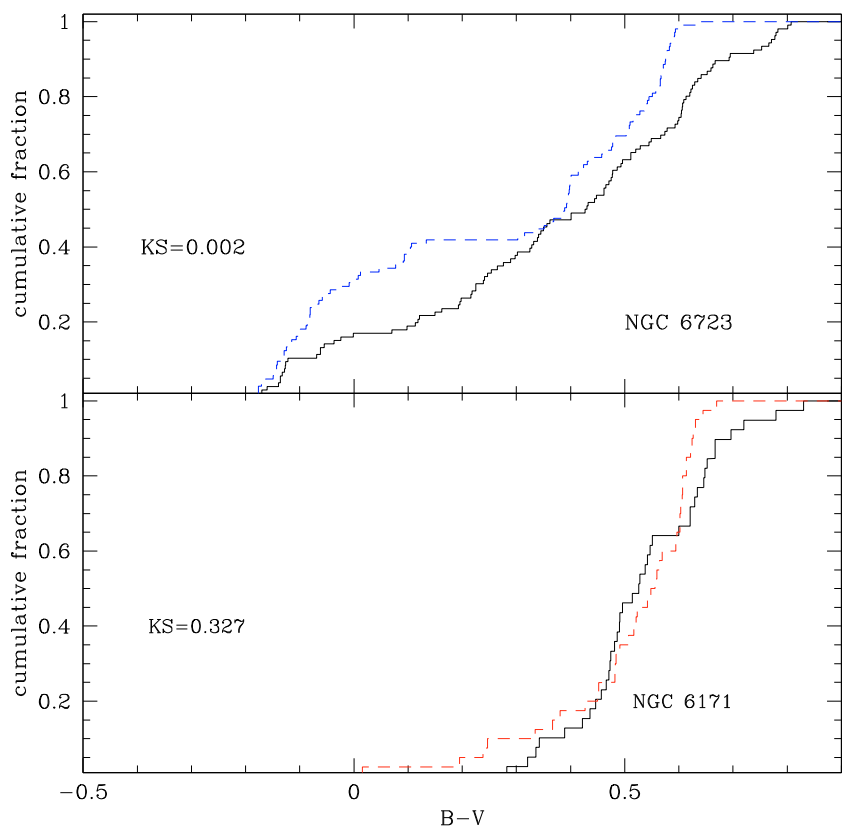

Fig. 25. As for Fig. 23, cumulative distributions and KS values for NGC 6171 and NGC 6723.

observed HB of NGC 6171 by a synthetic HB with a small Gaussian spread of $0.03 M_{\odot}$ in the mass lost (see Fig. 24). The shape of the colour distribution of NGC 6171 is not perfectly reproduced, again because the synthetic diagram predicts too many red stars, as shown by Fig. 25: however, given the small size of the sample, the result of the Kolmogorov-Smirnov test returns a $32.7 \%$ probability that observed and synthetic HBs are drawn from the same population, which is not significant. On the other hand, the distribution of colours of $\mathrm{HB}$ stars along the HB of NGC 6723 is clearly bimodal (see also Rood \& Crocker 1985), and there is no way of reproducing it assuming a Gaussian distribution of mass lost. In this case, the result of the Kolmogorov-Smirnov test is that the probability that a Gaussian distribution (with a dispersion of $0.07 M_{\odot}$ ) reproduces observations is $0.2 \%$. Hence, the case of the pair NGC 4833-NGC 5024 is not alone.

\subsection{The correlation between mass spread and absolute magnitude}

We propose that the cases discussed in the previous subsection, extracted from several decades of discussions, convincingly show the need for a third parameter in addition to metallicity and age to explain the blue extension of the HBs. Hereinafter, we quantify the spread in mass loss by the difference between the minimum and median mass of stars along the HB, and call this quantity $\delta M^{9}$. We note that $\delta M$ has been estimated by assuming a constant $\mathrm{He}$ abundance, hence it cannot be used directly to derive the spread in $\mathrm{He}$ within the cluster.

It has long been known that GCs with a large spread of masses along the HB are bright and massive (Fusi Pecci et al. 1993). Recio-Blanco et al. (2006) obtained a good correlation between the highest effective temperature along the HB

\footnotetext{
9 In principle, $M_{\max }$ should give a more correct estimate of the spread in mass along the HB. However, we preferred to use $M_{\text {med }}$ in our discussion for the reasons explained in Sect. 2.3, even though in some cases it may systematically underestimate the mass corresponding to stars with primordial He abundances
}

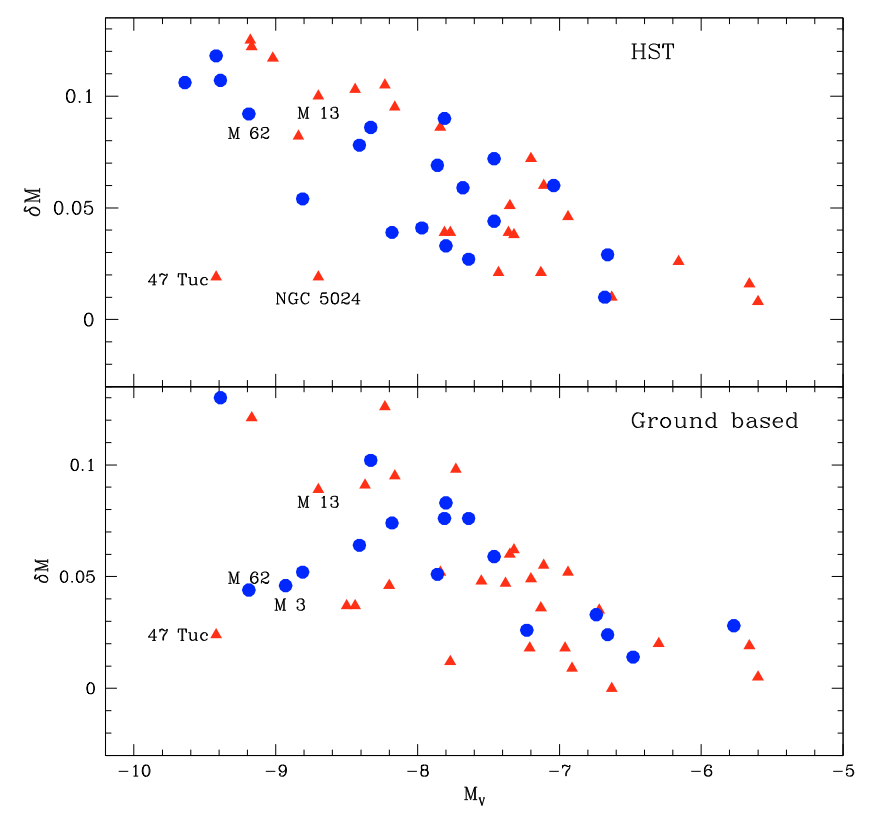

Fig. 26. Absolute magnitudes $\left(M_{\mathrm{V}}\right)$ plotted against the difference between the median and the minimum mass along the HB $(\delta M)$. In this case, we also separate GCs older (red triangles) and younger (blue filled dots) than 0.92 .

and the cluster absolute magnitude $M_{\mathrm{V}}$. Given that the highest effective temperature along the HB is well (anti-)correlated with the minimum mass of $\mathrm{HB}$ stars, we should expect a good anti-correlation between $\delta M$ and $M_{\mathrm{V}}$. In Fig. 26, we show the run of $\delta M$ with $M_{\mathrm{V}}\left(M_{\mathrm{V}}\right.$ values were taken directly from the Harris 1996 catalogue), for both the HST and ground-based data sets. There is indeed a very significant anti-correlation between these two quantities: large values of $\delta M$ are only found among bright clusters. The correlation is much cleaner if we restrict ourselves only to those GCs with age estimates, which have the highest quality colour-magnitude diagrams. Hereinafter, we use these GCs only, and combined ground-based and HST data. However, by comparing the two data sets, we noted that the anticorrelation between $\delta M$ and $M_{\mathrm{V}}$ is stronger when HST data are used ( $r=0.68$ for 45 clusters) than if ground-based data are considered ( $r=0.54$ for 46 clusters). This stronger correlation occurs because the extreme BHB stars are sometimes below the detection limit of the ground-based observations. Hence, when considering the two samples (whenever possible), we give preference to the HST data.

For this whole sample of 65 GCs (making up almost half of the total number of Galactic GCs), the linear relation between $\delta M$ and $M_{\mathrm{V}}$ is

$\delta M=-(0.102 \pm 0.026)-(0.020 \pm 0.003) M_{V} \quad M_{\odot}$

with an extremely significant correlation coefficient of $r=0.63$. The rms scatter of individual points along this mean relation $\left(0.026 M_{\odot}\right)$ is not much larger than expected from internal errors, suggesting that it is mainly due to observational errors. However, part of the scatter is certainly real. We note that there are a few clusters (namely NGC $104=47$ Tuc, NGC 5024; and to a lesser extent NGC 362 and NGC $5272=$ M 3 ) that have a small spread of masses along the $\mathrm{HB}$ in spite of there being quite massive; one of them has indeed been used in the comparisons of the previous subsection. There is little doubt that the spread in mass along the HB is small, at least for the two most extreme cases. We conclude that the additional parameter required to explain 
the extension of the $\mathrm{HB}$, while quite closely related to the overall cluster luminosity, is actually a separate one.

\subsection{He variations required explaining the $H B$ width}

In the remainder of this section, we examine the possibility that the additional parameter (to both metallicity and age) determining the spread in mass for stars along the HB is the variation in the He abundance, related to various stellar generations in GCs, which combines with a small $\left(\sim 0.02 M_{\odot}\right)$ random spread in mass loss. This last value was adopted because it is roughly the value required to explain those GCs with the minimum spread in mass for stars along the HB. In principle, this value may vary from cluster to cluster, depending e.g., on cluster concentration (see e.g., Fusi Pecci et al. 1993). However, we wish at present to keep our assumptions to a minimum.

To test the hypothesis presented above, we first derived the spread in He required to explain the observed spread in colours and masses along the HB. In the next section, we discuss the evidence provided by chemical abundances that might support this hypothesis. When deriving the spread in He, we should take into account that variations in $\mathrm{He}$ abundances have important effects on our analysis.

The first step of our procedure is quite simple. Since we assumed that an intrinsic spread in the mass loss equal to $0.02 M_{\odot}$ is a universal phenomenon, we corrected the observed mass spread for this effect by subtracting this value in quadrature from the observed $\delta M$. In those few cases where $\delta M<0.02$, we simply assumed that the corrected value is 0 . The corrected spread is then attributed to a variation in the He abundance, assuming that the same mass-loss law is applicable to all stars.

However, to derive the variation in $\mathrm{He}$, we should take into account that the masses derived for HB stars are themselves functions of the adopted He abundance. Unfortunately, the Pisa evolutionary tracks were computed for a single (not constant) value of the He abundance for each value of the metal abundance, and cannot then be used to estimate this correction. We therefore used instead the isochrones by Bertelli et al. (2008), which while assuming a unique value for the mass loss along the RGB (preventing its use to derive the relation between masses and colours along the HB), do however provide data for different He abundances. We then combined the two sets of models to produce the following correction formula for $\Delta M$

$\Delta M(Y)=\Delta M-\Delta Y\left(1.976+1.982[\mathrm{Fe} / \mathrm{H}]+0.562[\mathrm{Fe} / \mathrm{H}]^{2}\right)$.

In this equation, $\Delta Y$ is the variation in He abundance with respect to the reference value ( $Y=0.25$, roughly the cosmological value). In this case, we assume that all GCs started their evolution with an He abundance close to that resulting from the big bang nucleosynthesis ( $Y \simeq 0.25$, WMAP, Spergel et al. $2007-$ see their Table 4), but that there might be significant star-to-star variations even within the same GC. The procedure for estimating this He abundance is described in Sect. 5. Of course, the primordial He abundance in some clusters may actually be higher than the cosmological one. This is supported by both simple arguments based on a rather constant $\Delta Y / \Delta Z$ value throughout the Galactic evolution (Chiosi \& Matteucci 1982; Balser 2006; Casagrande et al. 2007), and indirect measurements in GC stars, e.g., those obtained using the $R$-method (see Sect. 5). However, these variations in primordial He are expected to be quite small (a few hundredths in $Y$ ), so that this assumption is not critical. In turn, $\Delta M(Y)$ may be derived from the evolutionary mass at the
Table 12. Spread in He and mass required explaining the HB.

\begin{tabular}{lcccccc}
\hline \hline Cluster & $\begin{array}{c}\delta M(Y) \\
M_{\odot}\end{array}$ & $Y_{\operatorname{med}}$ & $Y_{\max }$ & $\delta Y$ & $\begin{array}{c}\delta(B-V) \\
\text { mag }\end{array}$ & $\begin{array}{c}\delta(V-I) \\
\text { mag }\end{array}$ \\
\hline NGC 104 & 0.000 & 0.234 & 0.234 & 0.000 & 0.000 & 0.000 \\
NGC 288 & 0.016 & 0.280 & 0.292 & 0.012 & 0.005 & 0.007 \\
NGC 362 & 0.059 & 0.243 & 0.289 & 0.046 & 0.018 & 0.025 \\
NGC 1261 & 0.068 & 0.244 & 0.297 & 0.053 & 0.021 & 0.029 \\
NGC 1851 & 0.063 & 0.247 & 0.295 & 0.048 & 0.019 & 0.027 \\
\hline
\end{tabular}

Notes. The complete table is available at the CDS.

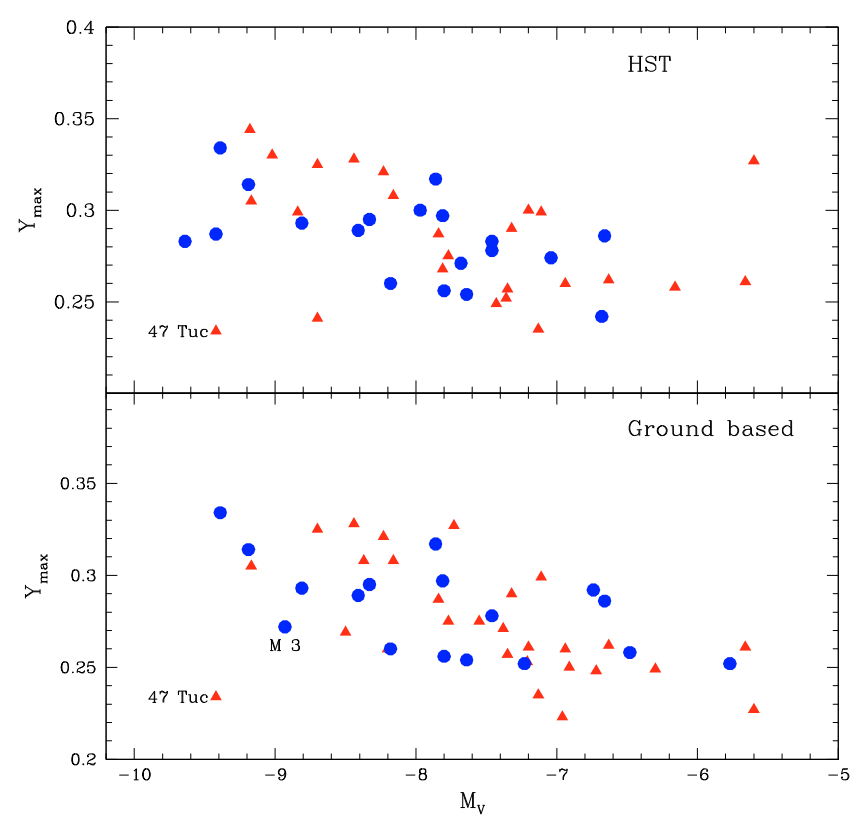

Fig. 27. Absolute magnitudes vs. $Y_{\max }$ for HST and ground-based samples; as in all previous figures, clusters are separated according to their ages.

tip of the RGB, corrected for the uniform mass-loss law along the RGB. In practice, we use the equation

$\Delta Y=\Delta M(Y) /\left(1.245+30.747 Z_{\odot} 10^{([m / H])}\right)$,

where $Z_{\odot}$ is the solar metal abundance, and $[\mathrm{m} / H]$ is the overall metallicity, which we assumed to be $[m / H]=[\mathrm{Fe} / \mathrm{H}]+0.28$, the second additive term taking into account the overall enhancement of $\alpha$-elements in GCs (see Carretta et al. 2009d). These two equations can be solved recursively, convergence being quite fast.

Table 12 lists the values of $Y_{\operatorname{med}}, Y_{\max }$, and $\delta Y=Y_{\max }-$ $Y_{\text {med }}$ obtained by this procedure for each cluster. We recall that in some cases $Y_{\text {med }}$ values might overestimate the minimum $Y$ in the cluster, and then underestimate $\delta Y$. The highest He abundances found using this procedure is $Y_{\max }=0.347$ for NGC 6273. The value we find for NGC 2808 is $Y_{\max }=0.333$; this value is discussed in the following section.

In Fig. 27, we plotted the $Y_{\max }$ values against the absolute magnitude $M_{\mathrm{V}}$. As expected, the correlation is good, yielding

$Y_{\max }=(0.180 \pm 0.025)-(0.0134 \pm 0.030) M_{V}$

with a very significant correlation coefficient of $r=0.49$ for 65 clusters. 


\subsection{Colour spread along the main sequence}

The spread in helium required to explain the spread in masses along the HB should cause a broadening of the MS. We estimated this expected colour spread in the MS at absolute magnitude $M_{\mathrm{V}}=+8$, by using the following formulae we derived by fitting data from the Bertelli et al. (2008) isochrones

$\delta(B-V)=\delta Y(0.350-0.035[\mathrm{Fe} / \mathrm{H}])$

and

$\delta(V-I)=\delta Y(0.672+0.099[\mathrm{Fe} / \mathrm{H}])$.

The colour spreads in $B-V$ and $V-I$ for each cluster are given in Cols. 6 and 7 of Table 12, respectively. Again, we recall that in some cases these spreads may be underestimated, because $\delta Y$ may itself be underestimated. This spread is very small, below detectability, for most of the GCs. The largest spread is expected for NGC 6273 (0.035 mag in $B-V$ and $0.043 \mathrm{mag}$ in $V-I$ ); this cluster is affected by a strong differential reddening, which complicates the detection of this spread.

Most GCs have not yet been scrutinised in enough detail, but in a few cases we may compare these predictions with observations. For instance, in the case of NGC 2808, Piotto et al. (2007) found a spread of $\sim 0.1 \mathrm{mag}$ in the $F 475 \mathrm{~W}-$ $F 814 \mathrm{~W}$ colour from very high quality ACS data. Since we expect that $\delta(F 475 W-F 814 W) / \delta(V-I) \sim 1.5$, the spread we predict from our analysis of the HB is $\delta(F 475 W-F 814 W) \sim 0.05$, which is roughly half the spread indeed observed. Part of this difference can be attributed to the median colour of the HB of NGC $2808\left(B-V_{\text {med }}=0.024\right)$ not corresponding to the red $\mathrm{HB}$, but rather to the $\mathrm{BHB}$, and in our framework is then interpreted as a moderately He enriched population $(Y=0.273)$. The He-poor population is however present, corresponding to the red HB, which makes up almost $40 \%$ of the cluster HB population. The total spread in colour along the MS is then expected to be larger than given simply by $\delta Y=Y_{\max }-Y_{\text {med }}$; a more appropriate estimate of the expected colour spread is $\delta(V-I) \sim 0.046$ and $\delta(F 475 W-F 814 W) \sim 0.07$. While this is still somewhat smaller than observed, the discrepancy is now small, and could be justified by some additional source of scatter (differential reddening, binaries, photometric errors) for MS colours or an incorrect calibration of the HB.

Anderson et al. (2009) found that some spread in colour is also present in 47 Tuc, from a very comprehensive analysis of extensive archive HST data. This spread is small, roughly $\delta(F 616 W-F 814 W) \sim 0.02$, and could only be identified thanks to the exceptional quality of the data, and the very careful procedures used to analyse them. However, the HB of 47 Tuc is very short ${ }^{10}$, so that our estimate for the He spread is only $\delta Y=0.0$ (based on the assumption that the small residual spread in masses could be explained by random star-to-star variations in the mass loss adopted throughout this paper), and we then expect no widening of the MS due to He. A careful study of the HB of 47 Tuc by Di Criscienzo et al. (2009) is work in progress; early results suggest that it may be more accurately represented by assuming that there is a very small spread in $Y(\delta Y \ll 0.02)$.

1047 Tuc actually contains a few very blue HB stars (Moehler et al. 2000); they make up 1\% of the HB stars in the HST colour-magnitude diagram we are considering. These stars are so rare that they do not affect our definition of $M_{\min }$, which excludes the lowest $5 \%$ of the distribution. Indeed, this case underlines that there most likely are stars at the extremes of the HB whose origin is not related to their extreme values of the He abundance.
By itself, this is not enough to justify the spread in colours of the MS. However, Di Criscienzo et al. also found that variations in the total CNO abundances might possibly explain both the small spread in colour of the MS and the far more evident split in the SGB, also found by Anderson et al. (2009; see however Bergbush \& Stetson 2009, for a different interpretation of this observation). While this comparison on the whole supports our result of a small spread in $\mathrm{He}$ in 47 Tuc, it also indicates that more careful examinations using synthesis of populations, taking into account the distribution in both colours and magnitudes of the stars on the HB, may provide additional important information about the properties of GCs. Extension of this careful analysis to many other GCs would be very helpful.

\section{He and GC chemistry}

\subsection{Light elements anti-correlations}

Additional evidence that the spread in colours along the HB of GCs is caused by variations in the He abundances can be obtained by considering correlations with similar spreads in the abundances of light elements such as $\mathrm{Na}$ and $\mathrm{O}, \mathrm{Mg}$ and $\mathrm{Al}$. As mentioned in the introduction, these spreads are caused by different generations of stars in GCs, the ejecta from the earliest one having polluted the material from which the later generation(s) of stars formed. While $p$-captures at high temperatures are clearly required to produce $\mathrm{Na}$ and $\mathrm{Al}$, and destroy $\mathrm{O}$ and $\mathrm{Mg}$, we do not yet have a satisfactory detailed model, and even its astrophysical basis is currently debated (either fast rotating massive stars or massive AGB stars, experiencing hot bottom burning, or massive binaries: see Decressin et al. 2007; Ventura et al. 2001; De Mink et al. 2009). We are unable to derive the exact mass range of the polluting stars. This is an important concern, because the production of He has probably a different dependence on the mass of the polluters than both the production of $\mathrm{Na}$ and the destruction of $\mathrm{O}$. For instance, if we consider the massive AGB scenario, $\mathrm{He}$ is mainly produced in the previous MS phase, and it is far more abundant in the ejecta of the most massive polluters (mass $>5 M_{\odot}$ ). On the other hand, a very significant production of $\mathrm{Na}$ and depletion of $\mathrm{O}$ can be obtained even within stars of lower mass. Hence, the ratio of $\mathrm{He}$ to $\mathrm{Na}$ production might change from cluster-to-cluster, provided that the mass range of polluters changes. With this caveat in mind, we can then examine current evidence.

In principle, the original $\mathrm{He}$ content should be attainable from direct measurements for each HB star. However, this datum is neither available nor can be easily obtained, save possibly for a restricted temperature range at $\sim 10000 \mathrm{~K}$ (Villanova et al. 2009; He is heavily depleted by sedimentation in warmer HB stars: see e.g., Behr et al. 2000b; Behr 2003). Furthermore, even $\mathrm{O}$ and $\mathrm{Na}$ abundances are not available for most $\mathrm{HB}$ stars (for a possible exception, see again Villanova et al. 2009), although in this last case abundances could in principle be obtained for stars on the RHB, within the instability strip, and on the coolest part of the BHB. Because of this shortage of data, we use values provided by RGB stars. Of course, the implication is that only statistical properties of the distributions, not individual values can be studied. In practice, we only consider extreme values, with the assumption, consistent with our approach, that the bluest (i.e., less massive) HB stars are the progeny of the most He-rich TOstars, and the reddest ones of the most He-poor ones; in a future paper, we plan to consider in more detail other characteristics of these distributions. 


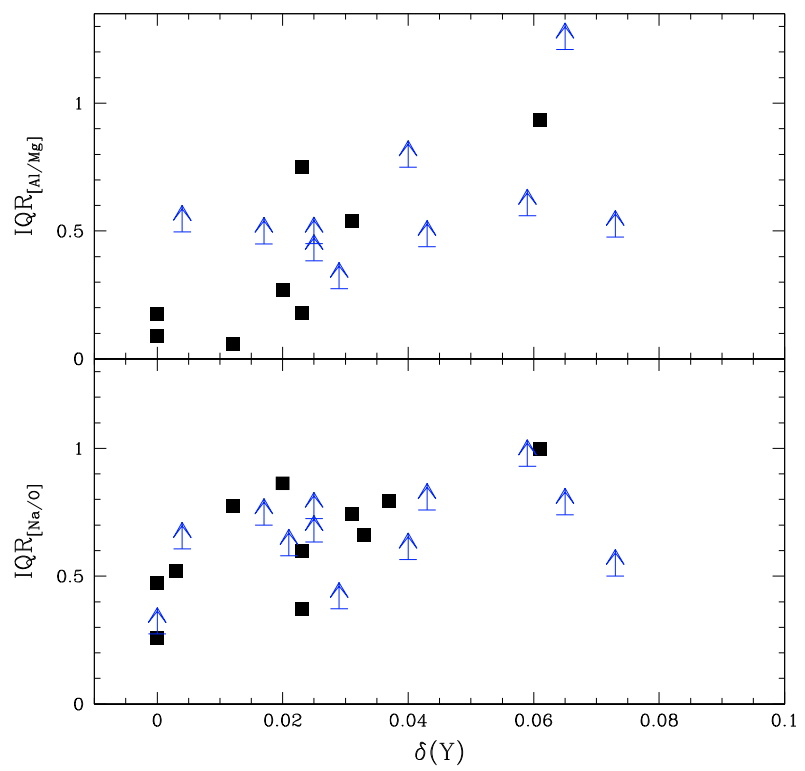

Fig. 28. $\delta Y$ as function of $\mathrm{IQR}$ for $[\mathrm{Na} / \mathrm{O}]$ and $[\mathrm{Al} / \mathrm{Mg}]$, respectively. Lower limits are also indicated.

In the present discussion, we considered a subset of the GCs with extensive enough data on the $\mathrm{Na}-\mathrm{O}$ and $\mathrm{Mg}$ - $\mathrm{Al}$ anticorrelations. Most of the GCs considered here are from the very extensive study by Carretta et al. (2009a,b); we complement this data set with a few GCs from the literature (Shetrone \& Keane 2000, for NGC 362; Sneden et al. 2004; and Cohen \& Melendez 2005, for NGC 5272 and NGC 6205; Sneden et al. 1991, 2000, for NGC 6341; Marino et al. 2008, for NGC 6121; Marino et al. 2009, for NGC 6656; and Yong et al. 2005, for NGC 6752). The relevant data are given in Table 1 . We note that we prefer to use the inter-quartile IQR of the distributions (either directly taken from the literature, or obtained from abundances for individual stars ${ }^{11}$ ), rather than the corresponding values for $90 \%$ of the distribution, as we did for the extension of the HB. The IQR is indeed a more robust indicator, which is less sensitive to small number statistics and to many upper limits to the abundance determinations. This last problem remains an important concern. In general, we may consider the IQR determinations as lower limits for those GCs with metallicity $[\mathrm{Fe} / \mathrm{H}]<-1.5$. Figure 28 illustrates the correlations between the spread in He abundances obtained from the colour spread of the HB, and the IQR values for both the $[\mathrm{Na} / \mathrm{O}]$ and the $[\mathrm{Al} / \mathrm{Mg}]$ anticorrelations. As can be seen, fairly tight correlations exist, the strongest being between $\delta Y$ and IQR([Na/O]), which are based on more extensive data sets than the IQR $([\mathrm{Al} / \mathrm{Mg}])$. This correlation strongly supports the current interpretation that the extent of the HB is determined by the spread in He content within each GC. However, we note that at least for $\delta Y-\mathrm{IQR}([\mathrm{Na} / \mathrm{O}]$, the relation has an offset, where $\delta Y$ is significantly larger than 0 only for clusters with $\operatorname{IQR}([\mathrm{Na} / \mathrm{O}]>0.6)$.

\subsection{Comparison with a dilution model}

We may compare these results with the prediction of a simple universal mechanism for the production of the $\mathrm{Na}-\mathrm{O}$ correlation, that has only one class of polluters, and for which the composition of second generation stars differ only in terms of the

\footnotetext{
11 We thank A. F. Marino for providing the unpublished data for the individual stars in NGC 6656.
}

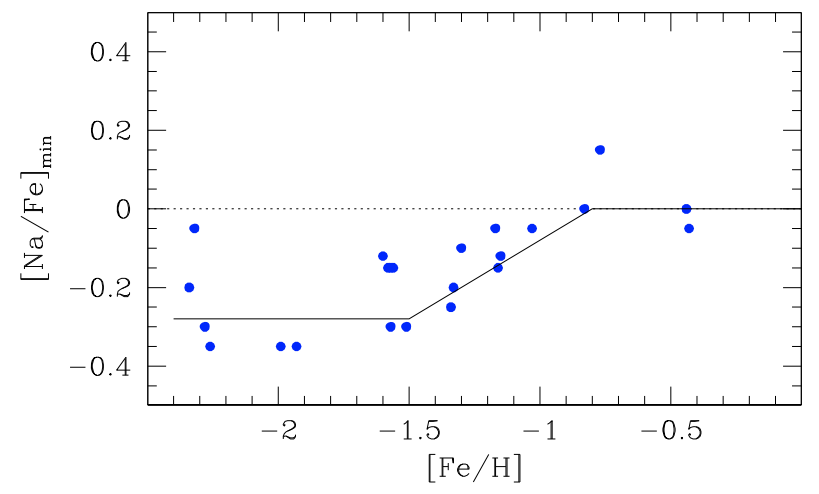

Fig. 29. Run of $[\mathrm{Na} / \mathrm{Fe}]_{\min }$ with $[\mathrm{Fe} / \mathrm{H}]$ for the GCs of our sample. The thick superimposed line represents an average line fitting field stars (see text)

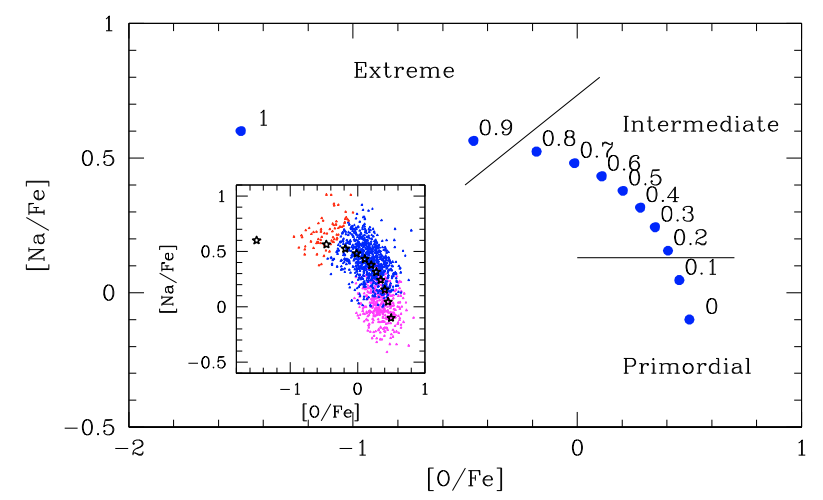

Fig. 30. Run of $[\mathrm{Na} / \mathrm{Fe}]$ vs. $[\mathrm{O} / \mathrm{Fe}]$ expected for different values of dil from our dilution model. The insert shows the run of $[\mathrm{Na} / \mathrm{Fe}]$ with [O/Fe] for stars in our FLAMES survey (Carretta et al. 2009a).

different dilution of polluted gas by primordial material. A similar dilution model has been successfully used to explain many features of the $\mathrm{Na}-\mathrm{O}$ anti-correlation (see discussion in Prantzos \& Charbonnel 2006), including its shape and the residual observed $\mathrm{Li}$ abundances in stars severely depleted in $\mathrm{O}$ in NGC 6752 (Pasquini et al. 2005). Once the compositions of the pristine and processed material are set (e.g., by the extremes of the observed distributions), the dilution factor may be determined for each star (either from $\mathrm{O}$ or $\mathrm{Na}$ abundances), and the helium production can be inferred, save for a constant factor.

In this model, the logarithmic abundance of an element $[X]$ for a given dilution factor $d i l$ is given by

$[X]=\log \left[(1-\operatorname{dil}) 10^{\left[X_{0}\right]}+\operatorname{dil} 10^{\left[X_{\mathrm{p}}\right]}\right]$,

where $\left[X_{\mathrm{o}}\right]$ and $\left[X_{\mathrm{p}}\right]$ are the logarithmic abundances of the element in the original and processed material, respectively. Practically speaking, we may assume (see Carretta et al. 2009a, Fig. 17) that the original composition is $[\mathrm{Na} / \mathrm{Fe}]=$ -0.28 if $[\mathrm{Fe} / \mathrm{H}]<-1.5,[\mathrm{Na} / \mathrm{Fe}]=0$ if $[\mathrm{Fe} / \mathrm{H}]>-0.8$, and $[\mathrm{Na} / \mathrm{Fe}]=-0.28+0.4([\mathrm{Fe} / \mathrm{H}]+1.5)$ if $-1.5<[\mathrm{Fe} / \mathrm{H}]<-0.8$; and $[\mathrm{O} / \mathrm{Fe}]=0.5$. For the processed material we assumed $[\mathrm{Na} / \mathrm{Fe}]=0.6$ and $[\mathrm{O} / \mathrm{Fe}]=-1.5$. We note that while for $\mathrm{O}$ we may assume a uniform $[\mathrm{O} / \mathrm{Fe}]$ value, because cluster-to-cluster variations of maximum values are small among the GCs of our sample, we adopted an original $[\mathrm{Na} / \mathrm{Fe}]$ ratio that is a function of metal abundance, to reproduce both our data and the $\mathrm{Na}$ abundances observed among field stars (see Fig. 29).

In Fig. 30, we compare the expected run of $[\mathrm{O} / \mathrm{Fe}]$ vs. $[\mathrm{Na} / \mathrm{Fe}]$ according to this dilution model, with observations for 
Table 13. Minimum and maximum values for $[\mathrm{Na} / \mathrm{Fe}]$ and $[\mathrm{O} / \mathrm{Fe}]$ (see text for references).

\begin{tabular}{lccccccc}
\hline \hline \multirow{2}{*}{ NGC } & \multicolumn{2}{c}{$[\mathrm{Na} / \mathrm{Fe}]$} & \multicolumn{2}{c}{$[\mathrm{O} / \mathrm{Fe}]$} & \multicolumn{2}{c}{ dil } & $\delta$ dil \\
& $\min$ & $\max$ & $\min$ & $\max$ & $\min$ & $\max$ & \\
& dex & dex & dex & dex & & & \\
\hline 104 & 0.15 & 0.74 & -0.40 & 0.38 & 0.14 & 0.87 & 0.73 \\
288 & -0.10 & 0.71 & -0.50 & 0.36 & 0.09 & 0.90 & 0.81 \\
362 & -0.15 & 0.40 & 0.10 & 0.38 & -0.00 & 0.51 & 0.51 \\
1904 & -0.15 & 0.72 & -0.60 & 0.28 & 0.12 & 0.93 & 0.81 \\
2808 & -0.12 & 0.56 & -1.00 & 0.37 & 0.02 & 0.99 & 0.97 \\
3201 & -0.30 & 0.60 & -0.80 & 0.32 & -0.02 & 0.97 & 0.99 \\
4590 & -0.35 & 0.53 & 0.00 & 0.72 & -0.05 & 0.62 & 0.67 \\
5272 & -0.15 & 0.55 & -0.10 & 0.50 & 0.12 & 0.70 & 0.58 \\
5904 & -0.25 & 0.60 & -0.70 & 0.43 & -0.03 & 0.95 & 0.98 \\
6121 & -0.05 & 0.74 & -0.20 & 0.37 & 0.08 & 0.77 & 0.69 \\
6171 & -0.05 & 0.69 & -0.30 & 0.39 & 0.03 & 0.83 & 0.80 \\
6205 & -0.12 & 0.70 & -1.00 & 0.50 & 0.15 & 0.99 & 0.84 \\
6218 & -0.20 & 0.67 & -0.40 & 0.56 & 0.01 & 0.87 & 0.86 \\
6254 & -0.30 & 0.56 & -0.40 & 0.47 & -0.02 & 0.87 & 0.89 \\
6341 & -0.30 & 0.45 & -0.10 & 0.38 & -0.02 & 0.70 & 0.72 \\
6388 & 0.00 & 0.67 & -0.60 & 0.24 & 0.00 & 0.93 & 0.93 \\
6397 & -0.35 & 0.71 & 0.00 & 0.37 & -0.05 & 0.62 & 0.67 \\
6441 & -0.05 & 0.80 & -0.40 & 0.20 & -0.04 & 0.87 & 0.91 \\
6752 & -0.15 & 0.65 & -0.40 & 0.53 & 0.12 & 0.87 & 0.75 \\
6809 & -0.35 & 0.69 & -0.20 & 0.44 & -0.05 & 0.77 & 0.82 \\
6838 & 0.00 & 0.76 & 0.00 & 0.48 & 0.01 & 0.62 & 0.61 \\
7078 & -0.05 & 0.70 & -0.10 & 0.49 & 0.23 & 0.70 & 0.47 \\
7099 & -0.20 & 0.76 & -0.20 & 0.60 & 0.07 & 0.77 & 0.70 \\
\hline
\end{tabular}

Notes. The dilution fraction is also reported.

the ensemble of stars observed within our project (Carretta et al. 2009a). It is important to notice that within this approach the production of $\mathrm{Na}$ (and of $\mathrm{He}$ ) saturates at large values of the dilution parameter dil. This has two important implications: (i) most reliable estimate of the maximum value of dil is given by the $\mathrm{O}$ abundances; and (ii) that similar He abundances are obtained for a rather wide range of $[\mathrm{O} / \mathrm{Fe}]$. On the other hand, the minimum value of dil is most accurately given by $\mathrm{Na}$ abundances, because similar values of $[\mathrm{O} / \mathrm{Fe}]$ are obtained for small values of dil. Minimum and maximum values of $\mathrm{O}$ and $\mathrm{Na}$ abundances measured in each cluster are given in Cols. 2-5 of Table 13. We note that while $\mathrm{Na}$ and $\mathrm{O}$ maximum abundances listed in this table are somewhat different from the values adopted in our dilution model, this has no consequences on our discussion because minimum and maximum values of the dil parameter (Cols. 6 and 7 of Table 13) are obtained from the minimum abundances of $\mathrm{Na}$ and $\mathrm{O}$, respectively. Both minimum and maximum values of dil contain some uncertainty, because only upper limits to $\mathrm{O}$ and $\mathrm{Na}$ abundances can be obtained in extreme cases; this caveat is stronger for metal-poor stars, and for the smallest GCs, where most of the observed stars are quite warm. For instance, we suspect that the minimum $\mathrm{Na}$ abundance is overestimated in the case of M 15, which is a very metal-poor GC, and that for this reason we underestimate the $\delta$ dil range appropriate for this cluster. Finally, the differences between the maximum and the minimum dilution are given in the last column of Table 13. Within this model, these values should be proportional to $\delta Y$.

We note that while this simple dilution model predicts quite uniform values of $\delta d i l$, and then $\delta Y$, the values we derived from the HB exhibit large variations from cluster-to-cluster. As a consequence, the correlation for individual clusters is poor. We may also remind that this simple model, which uses a universal polluter, also predicts that the $\mathrm{Na}-\mathrm{O}$ and $\mathrm{Mg}-\mathrm{Al}$ anticorrelations

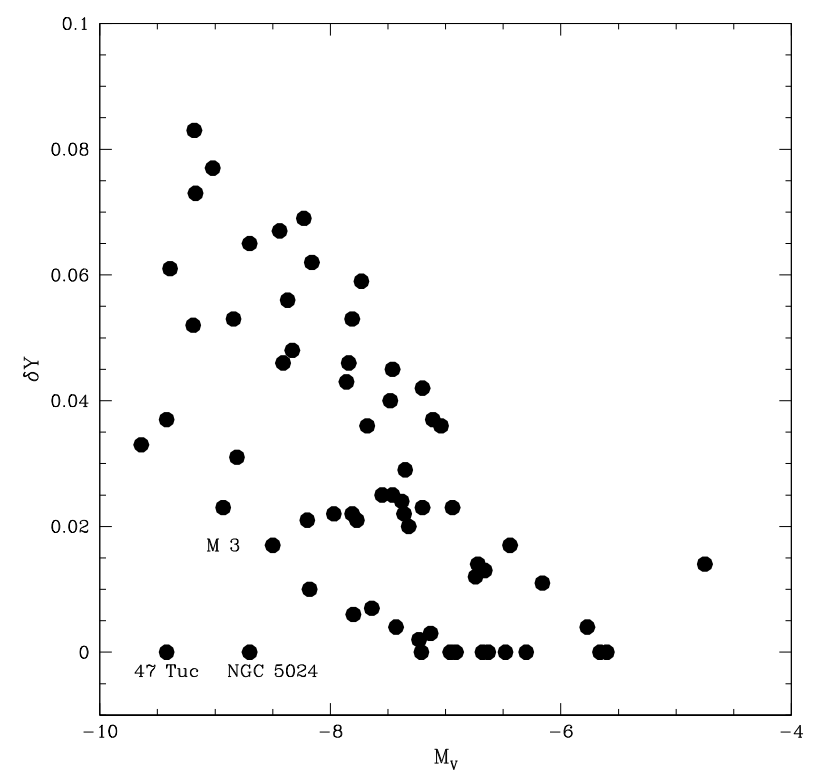

Fig. 31. Run of $\delta Y$ with absolute magnitude $M_{\mathrm{V}}$.

should closely resemble each other, which is not observed (see Carretta et al. 2009b).

We conclude that while a second generation of stars polluted by some stars of an earlier generation exists in all GCs, the composition of the polluters is not universal. In some clusters (such as NGC 2808), the polluters produce large amounts of $\mathrm{He}$ (most likely with similarly large amounts of $\mathrm{Al}$ ), and very efficiently destroy $\mathrm{O}$ and $\mathrm{Mg}$; while in others (like NGC 6121) practically no fresh $\mathrm{He}$ (and $\mathrm{Al}$ ) is present in the material that produced the second generation stars. On the other hand, all these polluters produce similar amounts of $\mathrm{Na}$. Independent of the polluter, we should be able to reproduce these observations, within a viable scenario for cluster formation and early evolution.

\subsection{The impact of cluster luminosity}

The previous discussion suggests that the spread in the $\mathrm{He}$ content within stars in GCs are the most important factor determining the extension of the HBs. It is important however to understand the basic physical property of GCs causing this spread in He. The existence of a good correlation between the maximum temperature of the $\mathrm{HB}$ and the cluster luminosity (Recio-Blanco et al. 2006) suggests that the total cluster mass determines its ability to have stars of very different He contents, assuming that the present cluster luminosity is a good proxy for its original mass. In Fig. 31 we show the correlation that we find between the spread in He abundance (as represented by $\delta Y$ ) and cluster luminosity $M_{\mathrm{V}}$, here a proxy for the total mass. There is a clear strong trend of an increase in $\delta Y$ with decreasing $M_{\mathrm{V}}$ (that is an increase in luminosity). The linear correlation coefficient is 0.56 for a total of 65 clusters, which is significant at a level of more than $99.99 \%$. The mean regression line is

$\delta Y=-(0.0127 \pm 0.0024) M_{V}-(0.069 \pm 0.020)$.

Although the correlation between the spread in He abundances (derived from the width of the HB) and luminosity is very good, there are a few clear exceptions, which are massive clusters with very short HBs (and hence small values of $\delta Y$ ), the clearest examples being NGC 104 (47 Tuc), and NGC 5024, and to a lesser extent NGC 5272 (M 3). We emphasize that the deviation of 
these clusters from the trend shown by the vast majority of the clusters is much larger than observational errors. It is notable that two of the most well studied clusters (47 Tuc and M 3), often considered as templates of metal-rich and metal-intermediate GCs for several studies of the HB, are found to be the exceptions rather than the rule. We recall that both NGC 104 and NGC 5272 have rather small values of IQR[Na/O], much less than observed in other clusters of similar luminosity. This again emphasizes the connection existing between the extension of the $\mathrm{HB}$ and the Na-O anticorrelation, hence supporting the explanation we suggest that the width of the HB is related to spreads in the He abundances.

We finally recall that D'Ercole et al. (2008) and Carretta et al. (2009d) presented a scenario for the formation of GCs in which the correlation between cluster luminosity and extension of its HB may occur naturally. According to that scenario, the formation of a GC is the final act of a series of events that begins from the violent onset of star formation in a very large original cloud. Star formation in this cloud continues until the kinetic energy injected by $\mathrm{SNe}$ and massive star winds causes dissipation of the remaining gas. In this scenario, this early population is not compact enough for the formation of a stable cluster, and should dissipate after the violent relaxation produced by the loss of the remaining gas and the ejecta of the most massive stars. However, once this very violent initial phase terminates, a much quieter situation follows, where there is no longer a strong injection of energy in the ISM; the slow winds from stars of intermediate mass may then lead to the onset of a cooling flow, and a formation of a very dense and kinematically cold cloud, from which the present GC may form, mainly composed by second generation stars (the I and E populations of Carretta et al. 2009a). Part of the primordial stellar population remains trapped in the current GCs, and forms what Carretta et al. (2009a) called the $P$-population. In this scenario, it is quite natural to expect that the onset of star formation within the cooling flow should occur earlier in more massive clusters; it will also probably stop earlier, because of the kinetic energy injected by the massive stars of this second generation. Therefore, we may expect that the delay of the second generation, hence the typical polluter mass, is roughly determined by the mass of the GC itself. A few exceptions may be easily accommodated in this scenario as objects characterised by a prolonged phase of formation of the primordial population, e.g., caused by the presence of a very extended region of star formation or companion clusters, as often observed among LMC populous clusters.

This scenario is clearly constructed on the hypothesis of massive AGB stars being polluters. Were rotating massive stars the polluters, some important modifications would be required, because these stars lose most of their mass in the epoch of corecollapse SNe explosions, and it would be very difficult to produce the required cooling flow. Reproducing the trends considered in this paper would clearly be a challenge for this alternative scenario.

\section{The M 3-M 13 pair}

Since the paper by Van den Bergh (1967), the pair M 3-M 13 (=NGC 5272 and NGC 6205) has played a very special rôle in the second parameter issue. This pair has been repeatedly studied to search for evidence of He-abundance variations (see Caloi \& D’Antona 2005; and Catelan et al. 2009-c). They are bright, massive and amongst the easiest GCs observable from the northern hemisphere. M 3, with its large population of RR Lyrae, has often been considered the archetype of Oo I GCs, while M 13 is considered to play the same rôle amongst the clusters with very blue HB. It is interesting to revisit them within the scenario outlined in this paper.

According to the basic parameters defined in this series of papers, M 3 and M 13 might not be so close twins, after all. While the metal abundance is quite similar $([\mathrm{Fe} / \mathrm{H}]=-1.50$ and -1.58 ), the ages might be different. Without considering the correction for the different $\mathrm{He}$ abundance, the relative ages we considered are 0.88 and 1.02 (see Table 1). After this correction is taken into account, this difference becomes even a bit larger (0.875 and 1.033). It is then possible that M 3 is younger then M 13, a result found in some careful determinations (see Rosenberg et al. 1999; and De Angeli et al. 2005), but not in others (Marín-Franch et al. 2009; Dotter et al. 2010). This by itself might explain why the median colour of the HB of M 13 is much bluer than that of M 3, without any need for a very large difference in $Y_{\text {med }}$ (we actually obtained values of 0.249 for M 3 and 0.260 for $M 13$ ).

However, it has for a long time been known that these two GCs also have a large difference in the extension of their HBs. According to our analysis, the spread in mass along the HB of M 3 is small. Assuming a constant helium abundance, we obtain minimum, median and maximum masses of $0.624 \pm 0.003$, $0.670 \pm 0.001$, and $0.716 M_{\odot}$, respectively. Apart from a general offset of about $0.02 M_{\odot}$ (which resembles the overall offset of masses derived from HST and ground-based data in our analysis), these values are quite similar to those obtained in the analysis of Valcarce \& Catelan (2008), although it is possible that some very blue/faint stars considered by these authors were actually not studied by the Rosenberg et al. (2000a) photometry. As discussed by several authors, the distribution of masses along the HB is probably not Gaussian (see Castellani et al. 2005; and Valcarce \& Catelan 2008). If the interpretation that we give for the mass spread along the HB in terms of variations in the He content is correct, we would correct the offset between median and minimum mass to $0.030 M_{\odot}$, and the corresponding spread in helium would be $\delta Y=0.023$. Catelan et al. (2009-c) argued that such a small spread in He in M 3 would be detectable from Strömgren photometry and spectroscopy of the blue HB stars, and concluded that a uniform He abundance more closely matches the observations for this cluster. This might suggest that small spreads in He such as the one we derived for M 3 might be an artifact of a non-Gaussian random mass-loss distribution. However, since the bluest HB stars $\left(T_{\text {eff }}>11500 \mathrm{~K}\right.$, consisting of some $5 \%$ of the whole HB population of M 3) could not be analyzed by Catelan et al., it is not entirely clear that there is no real spread in helium within M 3.

On the other hand, M 13 has a very wide range of both colours and masses along the HB. The spread in mass and $Y$ abundances we obtained using $M_{\text {med }}$ are $\delta M=0.082 M_{\odot}$ and $\delta Y=0.065$. Had we instead used $M_{\max }$, scaled down by $0.02 M_{\odot}$ to take into account the random term in mass lost, we would have got $\delta M=0.107 M_{\odot}$ and $\delta Y=0.083$. We expect then a detectable spread in colours for the MS of M 13: $[\delta(V-I)=0.033$ mag and possibly as much as $\delta(V-I)=0.043 \mathrm{mag}$, if $M_{\max }$ were adopted, assuming that the minimum $Y$ value is smaller than $Y_{\text {med }}$. This colour spread could possibly be detected in the future by a careful analysis of HST photometry.

As noticed by several authors, this huge difference in the width of the HB between M 3 and M 13 is quite unexpected, since $\mathrm{M} 3$ is a luminous cluster $\left(M_{\mathrm{V}}=-8.93\right)$, even more luminous than $\mathrm{M} 13\left(M_{\mathrm{V}}=-8.70\right)$. As the discussion in Sect. 6.4 shows, we should indeed expect a more extended HB for a luminous cluster like M 3. As a matter of fact, M 3 (and 
moreover 47 Tuc and NGC 5024) appears peculiar with respect to the other GCs, while M 13 appears to be similar to the majority of GCs in this respect. Within the scenario we described in the previous section (see also Carretta et al. 2009d), we are tempted to invoke a delayed cooling flow to explain the peculiarity of M 3 and 47 Tuc. This might explain other differences between M 3 and 47 Tuc on the one hand, and the other massive GCs on the other, such as the small values of IQR[Na/O]. However, we admit that without any further information, this hypothesis might look rather ad hoc ${ }^{12}$. Thus, ironically, while the present approach seems to explain most of the observations relate to the second parameter effect, its application to the most classical couple M 3-M 13 still encounters difficulties.

\section{Conclusions and suggestions for further work}

As we have seen, our reanalysis of public extensive photometric databases of GCs demonstrates that age is the main second parameter affecting the HB morphology. This hypothesis is able to explain quite well most of the observables related to median HB stars of GCs, when coupled with a simple mass-loss law that is a linear function of $[\mathrm{Fe} / \mathrm{H}]$. Among the many observables that are successfully explained, we note the Oosterhoff dichotomy that we attribute to the peculiar age-metallicity distribution of Galactic GCs. Oo II clusters are mostly old, while Oo I are predominantly young, although young Oo II and old Oo I GCs exist. However, at least a third parameter is required (and possibly even others) to fully explain the median colours of HBs (in particular those with very blue HBs) as well as their extension. There are various reasons to identify this third parameter with variations in the He content. These include the variation in the scatter with metallicity, some correlation with the $R$-parameter, and the clear links with chemical anomalies observed in GCs. This result is strongly indicative of a possible link between the colours of the stars on the HB and their original composition, in a multiple generation scenario for the formation and early evolution of GCs. Self-pollution in GCs is possibly responsible for a large variety of the second parameter features, and may be in part described using the $\mathrm{Na}-\mathrm{O}$ anticorrelation, although some modulation according to cluster luminosity is required ${ }^{13}$.

A combination of age and He variation therefore appears to be an explanation of the long-standing problem of the second parameter, although we do not exclude additional parameters such as the CNO abundances or the presence of binaries (e.g., of the progeny of blue stragglers) possibly playing some rôle. However, this issue is still far from being completely settled. We

\footnotetext{
12 After the first draft of this paper was written, Lane et al. (2010) suggested that 47 Tuc might have formed by the merging of a binary cluster. This suggestion comes from a completely different line of thought, being based on the internal kinematics. As mentioned in the previous section, it is possible to speculate on a possible connection between such an origin of 47 Tuc and its anomalously short HB.

13 The scenario we propose should of course not only explain the Milky Way GCs, but e.g., those of Fornax (Buonanno et al. 1998). This case is quite puzzling, since clusters 1,3 , and 5 are nearly coeval, and have similar metallicity, and still have very different HB's. We did not quantify these variations in terms of mean colours and magnitudes as done for the Milky Way GCs considered in the present paper, hence we cannot provide any quantitative analysis. We only note that the ranking of HBR ratios for the three coeval clusters of similar metallicity $(1,3$, and $5:-0.2,0.50$, and 0.44 ) is the same as the ranking in absolute magnitude $M_{\mathrm{V}}(-5.32,-7.66,-6.82)$. A quantitative analysis is required to settle this issue.
}

need to make some progress in developing models, and a number of observational tests. A short list includes:

- Understanding the nature of the polluters. This requires advances in the modeling of AGB stars and rotating massive stars. Furthermore, detailed spectroscopic data for stars in massive and very young clusters, such as RSGC1 and RSGC2 (Davies et al. 2007), or intermediate age clusters in the LMC, where multiple MS TO's have been observed (Mackey et al. 2008; Milone et al. 2009), may provide a crucial test of this scenario.

- The present discussion has focused on $\mathrm{He}, \mathrm{Na}$ and $\mathrm{O}$, but the abundances of other elements may also play an important rôle. For instance, Al might be a proxy for He that is more reliable than $\mathrm{Na}$. Unfortunately, our data for $\mathrm{Al}$ are not as extensive as those for $\mathrm{Na}$ and $\mathrm{O}$, but the relation between the production of $\mathrm{He}$ (which most likely occurred within MS stars), and the proton capture processes (which might have occurred in the same main sequence stars, if massive and fast rotating, or later during the AGB phase, if the stars were of intermediate mass) must be clarified.

- A number of confirmations of this scenario are required. These include (i) direct determinations of $\mathrm{He}, \mathrm{Na}$, and $\mathrm{O}$ in HB stars, which were shown to be possible in at least some cases by Villanova et al. (2009); and (ii) a discussion of the luminosity of the RGB bump that we defer to another paper currently in preparation.

- In addition, we ask: do properties of RR Lyrae variables agree with expectations? Are anti-correlations found where expected (important clusters such as M 54 and NGC 1851 do not yet have adequate data)? Are multiple sequences observed where they are expected? We note here that while the connections between multiple MSs and variations in the $\mathrm{He}$ abundance is quite clear, the case of multiple sub-giant branches (SGB) is more ambiguous. SGB splitting measured using visual-red-near infrared colours (see e.g., Milone et al. 2008) might be due to a variation either in age or most likely total CNO content (see e.g., D'Antona et al. 2009; and Cassisi et al. 2008), or even [Fe/H] (in this case however some spread in the MS and RGB is also expected). Interpretation of splitting is even more ambiguous when considering ultraviolet colours, which are sensitive to $\mathrm{N}$ excesses. Variations in He abundances are only marginally effective in these cases, because sequences differing only in $Y$ are very close each other on the SGB (D'Antona et al. 2002). Variations in total CNO content can be most likely attributed to the contribution of thermally pulsing AGB stars (Cassisi et al. 2008), which have a rather low mass and probably do not contribute much to $\mathrm{He}$ abundance variations. It is then unclear that there should be any correlation between the SGB splitting and large spreads on the HBs. In fact, NGC 2808 has a quite narrow SGB (Piotto et al. 2007). SGB splitting has been detected using visual-red-near infrared colours in 47 Tuc (Anderson 2009), NGC 1851 (Milone et al. 2008), and NGC 6388 (Moretti et al. 2009). These clusters have very different $\mathrm{HB}$ morphologies, ranging from very short (47 Tuc), to bimodal (NGC 1851), to very extended (NGC 6388). We obtain very different estimates of the He spread ( 0 for 47 Tuc; 0.048 for NGC 1851, a possibly too large value compared with those determined by Salaris et al. 2008; and Catelan et al. 2010; 0.037 for NGC 6388). This lack of correlation suggests that the two phenomena are somewhat different, as expected if the mass range of the polluters changes from cluster-to-cluster. 
- The scenario requires a number of refinements. All analytic dependencies adopted throughout this discussion should be reviewed, and possibly replaced by a comparison with synthetic HBs. This may allow us to detect additional effects, e.g., a variation in total $\mathrm{CNO}$ abundances, not included in the present analysis.

- Finally, hydrodynamical simulations of the formation and early evolution of massive star clusters are urgently needed. There are aspects of the present scenario that are necessary to explain observations, but should be understood more clearly. The most intriguing is the existence of a pool of gas from which second generation stars formed, which is composed of material processed through H-burning at high temperature diluted with pristine gas. How this pool of gas is generated, and how the stars form from it within the potential well of the young GC, while other stars of the original population evolve, remains unclear. Some explorative results were obtained by D'Ercole et al. (2008) which while very promising should be placed on a sounder basis.

Acknowledgements. The authors wish to thank the anonymous referee for insightful and useful comments which lead to a significant improvement of the paper. L. Girardi for having provided the results of the TRILEGAL simulations used for field decontamination. F. D'Antona and S. Cassisi deserve to be acknowledged for a critical reading of the manuscript and for the very valuable suggestions. Finally, we thank Mariangela Bonavita for help in the preparation of the figures, and the Italian MUR for financial support through PRIN 20075TP5K9. This research has made use of NASA's Astrophysics Data System.

\section{References}

Anderson, J., Piotto, G., King, I. R., Bedin, L. R., \& Guhathakurta, P. 2009, ApJ, 697, L58

Balser, D. S. 2006, AJ, 132, 2326

Bedin, L., Piotto, G., Anderson, J., et al. 2004, ApJ, 605, L125

Behr, B. B. 2003, ApJS, 149, 67

Behr, B. B., Djorgovski, S. G., Cohen, J. G., et al. 2000a, ApJ, 528, 849

Behr, B. B., Cohen, J. G., \& McCarthy, J. K. 2000b, ApJ, 531, L37

Bergbusch, P. A., \& Stetson, P. B. 2009, AJ, 138, 1455

Bertelli, G., Girardi, L., Marigo, P., \& Nasi, E. 2008, A\&A, 484, 815

Buonanno, R., Corsi, C. E., Zinn, R., et al. 1998, ApJ, 501, L33

Caloi, V., \& D'Antona, F. 2005, A\&A, 435, 987

Caloi, V., \& D'Antona, F. 2007, A\&A, 463, 949

Caloi, V., \& D'Antona, F. 2008, ApJ, 673, 847

Cariulo, P., Degl'Innocenti, S., \& Castellani, V. 2004, A\&A, 421, 1121

Carretta, E., Recio-Blanco, A., Gratton, R. G., Piotto, G., \& Bragaglia, A. 2007, ApJ, 671, L125

Carretta, E., Bragaglia, A., Gratton, R. G., et al. 2009a, A\&A, 505, 117

Carretta, E., Bragaglia, A., Gratton, R. G., \& Lucatello, S. 2009b, A\&A, 505, 139

Carretta, E., Bragaglia, A., Gratton, R. G., D’Orazi, V., \& Lucatello, S. 2009c, A\&A, 508, 695

Carretta, E., Bragaglia, A., Gratton, R. G., et al. 2009d, A\&A, accepted

Casagrande, L., Flynn, C., Portinari, L., Girardi, L., \& Jimenez, R. 2007, MNRAS, 382, 1516

Cassisi, S., Castellani, V., degl'Innocenti, S., \& Weiss, A. 1998, A\&AS, 129, 267

Cassisi, S., Salaris, M., \& Irwin, A. W. 2003, ApJ, 588, 862

Cassisi, S., Salaris, M., Pietrinferni, A., et al. 2008, ApJ, 672, L115

Castellani, V., Degl'Innocenti, S., Marconi, M., Prada Moroni, P. G., \& Sestito, P. 2003, A\&A, 404, 645

Castellani, M., Castellani, V., \& Cassisi, S. 2005, A\&A, 437, 1017

Catelan, M. 2009a, Resolved Stellar Populations, ASP Conf. Ser., ed. D. Valls-Gabaud, \& M. Chavez, Ap\&SS, 320, 261

Catelan, M. 2009b, in The Ages of Stars: The horizontal branch, IAU Symp., 258, 209

Catelan, M., \& de Freitas Pacheco, J. A. 1995, A\&A, 297, 345

Catelan, M., Borissova, J., Sweigart, A. V., \& Spassova, N. 1998, ApJ, 494, 265

Catelan, M., Bellazzini, M., Landsman, W. B., et al. 2001, AJ, 122, 3171

Catelan, M., Grundahl, F., Sweigart, A. V., Valcarce, A. A. R., \& Cortés, C. 2009, ApJ, 695, 97
Catelan, M., Valcarce, A. A. R., Sweigart, A. V. 2010, in Star clusters: basic galactic building blocks throughout time and space, IAU Symp., 266, 281

Chiosi, C., \& Matteucci, F. 1982, A\&A, 105, 140

Clement, C. M., Muzzin, A., Dufton, Q., et al. 2001, AJ, 122, 2587

Code, A. D. 1969, PASP, 81, 475

Cohen, J. G., \& Melendez, J. 2005, AJ, 129, 303

Cohen, J. G., Briley, M. M., \& Stetson, P. B. 2002, AJ, 123, 2525

Contreras, R., Catelan, M., Smith, H. A., Pritzl, B. J., \& Borissova, J. 2005, ApJ, 623, L117

Corwin, T. M., Catelan, M., Borissova, J., \& Smith, H. A. 2004, A\&A, 421, 667

D’Antona, F., \& Caloi, V. 2004, ApJ, 611, 871

D'Antona, F., \& Caloi, V. 2008, MNRAS, 390, 693

D'Antona, F., Caloi, V., Montalban, J., Ventura, P., \& Gratton, R. 2002, A\&A, 395, 69

D’Antona, F., Bellazzini, M., Caloi, V., et al. 2005, ApJ, 631, 868

D'Antona, F., Stetson, P. B., \& Ventura, P. 2009, MNRAS, 399, 151

Davies, B., Figer, D. F., Kudritzki, R.-P., et al. 2007, ApJ, 671, 781

D’Cruz, N. L., Dorman, D., Rood, R. T., \& O'Connell, R. W. 1996, ApJ, 466, 359

de Angeli, F., Piotto, G., Cassisi, S., et al. 2005, AJ, 130, 116

Decressin, T., Meynet, G., Charbonnel, C., Prantzos, N., \& Ekstrm, S. 2007, A\&A, 464, 1029

Deliyannis, C. P., Demaruqe, P., \& Pinsonneault, M. H. 1989, ApJ, 347, L73

Demarque, P., Lee, Y.-W., Zinn, R., \& Green, E. M. 1989, IAU Meeting JCM 5 and $\mathrm{CM} 37 / 3$, The abundance spread within globular clusters: spectroscopy of individual stars, 97

De Minck, S. E., Pols, O. R., Langer, N., \& Izzard, R. G. 2009, A\&A, 507, L1

D’Ercole, A., Vesperini, E., D’Antona, F., McMillan, S. L. W., \& Recchi, S. 2008, MNRAS, 391, 825

Di Criscienzo, M., Marconi, M., \& Caputo, F. 2004, ApJ, 612, 1092

Dotter, A. 2008, ApJ, 687, L21

Dotter, A., Sarajedini, A., \& Yang, S. C. 2008, AJ, 136, 1407

Dotter, A., Sarajedini, A., Anderson, J., et al. 2010, ApJ, 708, 698

Dupree, A. K., Smith, G. H., Strader, J., et al. 2009, AJ, 138, 1485

Faulkner, J. 1966, ApJ, 144, 978

Ferraro, F. R., Clementini, G., Fusi Pecci, F., Buonanno, R., \& Alcaino, G. 1990, A\&AS, 84, 59

Freeman, K. C., \& Norris, J. 1981, ARA\&A, 19, 319

Fusi Pecci, F., Ferraro, F. R., Bellazzini, M., et al. 1993, AJ, 105, 1145

Fusi Pecci, F., Bellazzini, M., Cacciari, C., \& Ferraro, F. R. 1995, AJ, 110, 1664

Girardi, L., Groenewegen, M. A. T., Hatziminaoglou, E., \& da Costa, L. 2005, A\&A, 436, 895

Goldberg, L. 1979, QJRAS, 20, 36

Gratton, R. G., Bonifacio, P., Bragaglia, A., et al. 2001, A\&A, 369, 87

Gratton, R. G., Sneden, C., \& Carretta, E. 2004, ARA\&A, 42, 385

Green, et al. 1997, in Third Conference on Faint Blue Stars, 271

Han, Z., Podsiadlowski, P., Maxted, P. F. L., \& Marsh, T. R. 2003, MNRAS, 341, 669

Harris, W. E. 1996, AJ, 112, 1487

Harris, W. E., Bell, R. A., Vandenberg, D. A., et al. 1997, AJ, 114, 1030

Iben, I., Jr. 1968, Nature, 220, 143

Iben, I., Jr, \& Rood, R. T. 1970, ApJ, 159, 605

Judge, P. G., \& Stencel, R. E. 1991, ApJ, 371, 357

Kraft, R. P. 1994, PASP, 106, 553

Lane, R. R., Brewer, B. K., Kiss, L. L., et al. 2010, ApJ, 711, L122

Lee, Y.-W., \& Zinn, R. 1990, in Confrontation between Stellar Pulsation and Evolution, ed. C. Cacciari, \& G. Clementin (San Francisco: ASP), ASP Conf. Ser., 11, 26

Lee, J.-W., \& Carney, B. 1999, AJ, 118, 1373

Lee, Y.-W., Demarque, P., \& Zinn, R. 1990, ApJ, 350, 155

Lee, Y.-W., Demarque, P., \& Zinn, R. 1994, ApJ, 423, 248

Lee, Y.-W., Joo, S.-J., \& Han, S.-I. 2005, ApJ, 621, L57

Mackey, A. D., Broby Nielsen, P., Ferguson, A. M. N., \& Richardson, J. C. 2008, ApJ, 681, L17

Marcolini, A., Gibson, B. K., Karakas, A. I., \& Sanchez-Blazquez, P. 2009, MNRAS, 395, 719

Marconi, M., Caputo, F., Di Criscienzo, M., \& Castellani, M. 2003, ApJ, 596, 299

Marín-Franch, A., Aparicio, A., Piotto, G., et al. 2009, ApJ, 694, 1498

Marino, A. F., Villanova, S., Piotto, G., et al. 2008, A\&A, 490, 625

Marino, A. F., Milone, A., Piotto, G., et al. 2009, A\&A, 505, 1099

Maxted, P. F. L., Heber, U., Marsh, T. R., \& North, R. C. 2001, MNRAS, 326, 1391

Mészáros, Sz., Avrett, E. H., \& Dupree, A. K. 2009, AJ, 138, 615

Milone, A. P., Bedin, L. R., Piotto, G., Anderson, J., \& King, I. R. 2008, ApJ, 673,241

Milone, A. P., Bedin, L. R., Piotto, G., \& Anderson, J. 2009, ApJ, 497, 755

Milone, A. P., Piotto, G., King, I. R., et al. 2010, ApJ, 709, 1183 
R. G. Gratton et al.: The second and third parameters of the horizontal branch

Moehler, S. 2001, PASP, 113, 1162

Moehler, S., Landsman, W. B., \& Dorman, B. 2000, A\&A, 361, 937

Moni Bidin, C., Moehler, S., Piotto, G., et al. 2006, A\&A, 451, 499

Moretti, A., Piotto, G., Arcidiacono, C., et al. 2009, A\&A, 539, 546

Mullan, D. J. 1978, ApJ, 226, 151

Napiwotzki, R., Karl, C. A., Lisker, T., et al. 2004, Ap\&SS, 291, 321

Norris, J. E. 1981, ApJ, 248, 177

Norris, J. E. 2004, ApJ, 612, L25

Oosterhoff, P. Th. 1944, BAN, 10, 550

Origlia, L., Rood, R. T., Fabbri, S., et al. 2007, ApJ, 667, L85

Origlia, L., et al. 2008, MSAIt, 79, 432

Pasquini, L., Bonifacio, P., Molaro, P., et al. 2005, A\&A, 441, 549

Peterson, R. 1982, ApJ, 258, 499

Piotto, G., King, I. R., Djorgovski, S. G., et al. 2002, A\&A, 391, 945

Piotto, G., Villanova, S., Bedin, L. R., Gratton, R., \& Cassisi, S. 2005, ApJ, 621, 777

Piotto, G., Bedin, L. R., Anderson, J., et al. 2007, ApJ, 661, L53

Prantzos, N., \& Charbonnel, C. 2006, A\&A, 458, 135

Pritzl, B. J., Smith, H. A., Catelan, M., \& Sweigart, A. V. 2002, AJ, 124, 949

Pritzl, B. J., Smith, H. A., Stetson, P. B., et al. 2003, AJ, 126, 1381

Pritzl, B. J., Armandroff, T. E., Jacoby, G. H., \& Da Costa, G. S. 2004, AJ, 127, 318

Recio-Blanco, A., Piotto, G., Aparicio, A., \& Renzini, A. 2002, ApJ, 572, L71

Recio-Blanco, A., Aparicio, A., Piotto, G., de Angeli, F., \& Djorgovski, S. G. 2006, A\&A, 452, 875

Reimers, D. 1975a, in Problems in Stellar Atmospheres and Envelopes, ed. B. Baschek, W. H. Kegel, \& G. Traving (Berlin: Springer), 229

Reimers, D. 1975b, Mem. Soc. R. Sci. Liège 6 Ser., 8, 369

Renzini, A. 1977, in Advanced Stages in Stellar Evolution, ed. P. Bouvier, \& A. Maeder, Obs. de Geneve, Geneve, 151

Rich, M. R., Sosin, C., Djorgovski, S. G., et al. 1997, ApJ, 484, L25

Rood, R. T. 1973, ApJ, 184, 815

Rood, R. T., \& Crocker, D. A. 1985, in Horizontal-branch and UV-bright stars, 99

Rosenberg, A., Saviane, I., Piotto, G., \& Aparicio, A. 1999, AJ, 118, 2306
Rosenberg, A., Piotto, G., Saviane, I., \& Aparicio, A. 2000a, A\&AS, 144, 5 Rosenberg, A., Aparicio, A., Saviane, I., \& Piotto, G. 2000b, A\&AS, 145, 451

Salaris, M., Riello, M., Cassisi, S., \& Piotto, G. 2004, A\&A, 420, 911

Salaris, M., Cassisi, S., \& Pietrinferni, A. 2008, ApJ, 678, L25

Sandage, A. R. 1982, ApJ, 252, 553

Sandage, A., \& Wallerstein, G. 1960, ApJ, 131, 598

Sandage, A., \& Wildey, R. 1967, ApJ, 150, 469

Sandquist, E. L., Taam, R. E., \& Burkert, A. 2000, ApJ, 533, 984

Sarajedini, A., Bedin, L. R., Chaboyer, B., et al. 2007, AJ, 133, 1658

Shetrone, M. D., \& Keane, M. J. 2000, AJ, 119, 840

Sneden, C., Kraft, R. P., Prosser, C. F., \& Langer, G. E. 1991, AJ, 102, 2001

Sneden, C., Pilachowski, C. A., \& Kraft, R. P. 2000, AJ, 120, 1351

Sneden, C., Kraft, R. P., Guhathakurta, P., Peterson, R. C., \& Fulbright, J. P. 2004, AJ, 127, 2162

Soker, N., \& Harpaz, A. 2000, MNRAS, 317, 861

Spergel, D. N., Bean, R., Dorè, O., et al. 2007, ApJ, 170, 377

Stephens, A. W., \& Frogel, J. A. 2004, AJ, 127, 925

Stetson, P. B., Vandenberg, D. A., \& Bolte, M. 1996, PASP, 108, 560

Sweigart, A. V. 2002, Highlights in Astronomy, 12, 292

Sweigart, A. V., \& Gross, P. G. 1976, ApJS, 32, 367

Szczygiel, D. M., Pojmanski, G., \& Pilecki, B. 2009, AcA, 59, 137

Valcarce, A. A. R., \& Catelan, M. 2008, A\&A, 487, 185

van den Bergh, S. 1967, AJ, 72, 70

VandenBerg, D. A., Swenson, F. J., Rogers, F. J., Iglesias, C. A., \& Alexander, D. R. 2000, ApJ, 532, 430

Vanhollebeke, E., Groenewegen, M. A. T., \& Girardi, L. 2009, A\&A, 498, 95

Ventura, P., D’Antona, F., Mazzitelli, I., \& Gratton, R. 2001, ApJ, 550, L65

Villanova, S., Piotto, G., \& Gratton, R. G. 2009, A\&A, 499, 755

Willson, L. A. 2000, ARA\&A, 38, 573

Yong, D., Grundahl, F., Nissen, P. E., Jensen, H. R., \& Lambert, D. L. 2005, A\&A, 438, 875

Zinn, R. 1980, ApJ, 241, 602

Zinn, R. 1985, ApJ, 293, 424

Zoccali, M., Cassisi, S., \& Bono, G. 2000, ApJ, 538, 289 